SLAC-349

$\mathrm{UC}-34 \mathrm{D}$

(T)

\title{
TWO ASPECTS OF ONE LOOP STRUCTURE: UNITARITY DELAY IN THE STANDARD MODEL AND MODULAR INVARIANCE IN STRING THEORY*
}

\author{
$-$ \\ Changrim Ahn \\ Stanford Linear Accelerator Center \\ Stanford University \\ Stanford, California 94309
}

August 1989

Prepared for the Department of Energy under contract number DE-AC03-76SF00515

Printed in the United States of America. Available from the National Technical Information Service, U.S. Department of Commerce, 5285 Port Royal Road, Springfield, Virginia 22161. Price: Printed Copy A06, Microfiche A01.

\footnotetext{
* Ph.D. thesis
} 


\begin{abstract}
We study two aspects of one loop structures in quantum field theories which describe two different areas of particle physics: the one loop unitarity behavior of the Standard Model of electroweak interactions and modular invariance of string model theory. Loop expansion has its importance in that it contains quantum fluctuations due to all physical states in the theory. Therefore, by studying the various models to one loop, we can understand how the contents of the theory can contribute to physically measurable quantities and how the consistency at quantum level restricts the physical states of the theory, as well.
\end{abstract}

In the first half of the thesis, we study one loop corrections to the process $e^{+} e^{-} \rightarrow W^{+} W^{-}$. In this process, there is a delicate unitarity-saving cancellation between s-channel and t-channel tree level Feynman diagrams. If the one loop contribution due to heavy particles corrects the channels asymmetrically, the cancellation, hence unitarity, will be delayed upto the mass scale of these heavy particles. We refer to this phenomena as the unitarity delay effect. Due to this effect, cross section below these mass scales can have significant radiative corrections which may provide an appropriate window through which we can see the high energy structure of the Standard Model from relatively low energy experiments.

In the second half, we will show how quantum consistency can restrict the physical states in string theory. Despite the absence of a complete formulation of string field theory, it is known that conventional Feynman loop diagrams of point field theory generalize to the two dimensional Riemann surface. Modular 
transformations are symmetries of the Riemann surface and, therefore, a physical amplitude should be invariant under this operation. The zero-point ampitude on the torus can be interpreted as the partition function of the underlying two dimensional conformal field theory. Modular invariance of the partition function plays the role of a selection rule for the allowable physical spectrum of the conformal field theory. Complete classifications of modular invariant partition functions for general conformal field theories are important unsolved problems because they serve both as the classical vacuua of string theory and as systems in statistical mechanics at their critical points. We provide a method to derive modular invariant partition functions for Wess-Zumino-Witten models of general group manifolds. using the orbifold construction. When we add both the twisted and untwisted sectors correctly, we obtain the modular invariant partition functions on non-simply connected group manifolds. 


\section{ACKNOWLEDGEMENTS}

I would like to thank the members of my committee, Savas Dimopoulos, Fred Gilman, Walter Toki, and my advisor, Michael Peskin. Their patient guidance and encouragement were essential for this work. In particular, I will remain deeply grateful to my advisor throughout my career as a physicist. I am also grateful to my collaborators, Bryan Lynn and Mark Walton.

I am indebted to too many people to acknowledge them all. I am especially thankful for the SLAC theory group and the warm atmosphere it provided a foreign student. Many thanks to my friends and colleagues for their friendship. I would also like to thank my parents and my relatives, especially Mr.Jae Jun Ahn. Without their help, I would not be here.

Finally, I dedicate this thesis to my mother who is alive always in my mind. 


\section{TABLE OF CONTENTS}

I. INTRODUCTION . . . . . . . . . . . . . . . . . . . 1

1. Loop Expansion . . . . . . . . . . . . . . . . . . . . . 2

2. Unitarity Delay _................... 5

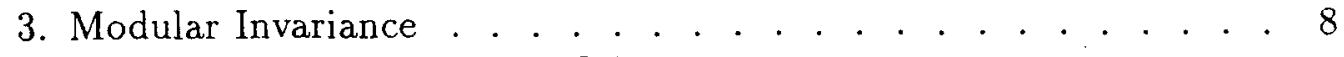

II. DELAYED UNITARITY CANCELLATION

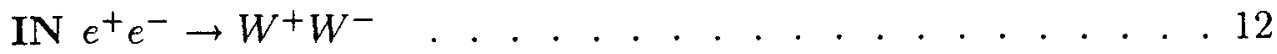

1. Introduction . . . . . . . . . . . . . . . 13

2. General Formalism . . . . . . . . . . . . 17

3. One-Loop Radiative Corrections . . . . . . . . . . . . . 26

4. Low and High Energy Behavior . . . . . . . . . . 36

5. Numerical Results and Discussion . . . . . . . . . . 40

6. Conclusion . . . . . . . . . . . . . 50

APPENDIX A: Computation of Differential Cross Sections . . . . 51

APPENDIX B: Explicit Formulae for the $W^{+} W^{-}$Form Factors . . . 53

APPENDIX C: Reduced Passarino-Veltman Functions . . . . . . . 58

III. MODULAR INVARIANT PARTITION FUNCTIONS

ON NONSIMPLY CONNECTED GROUP MANIFOLDS . . 64 
1. Preliminaries ................ . . . 65

2. Kač-Moody Algebra . . . . . . . . . . . . . . . 75

3. Modular Invariant Partition Functions from Orbifolds . . . . . . 81

4. Further Remarks . . . . . . . . . . . . . . . . .91

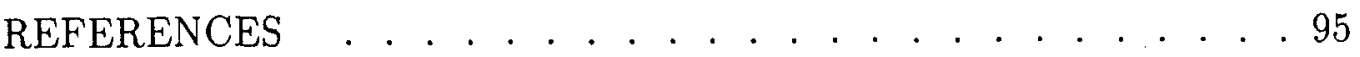

\section{LIST OF FIGURES}

Figure 1: The general vertex for $W$ pairs $\ldots \ldots . . . .18$

Figure 2: The amplitude for $e^{+} e^{-} \rightarrow W^{+} W^{-} \quad \ldots . . \quad . \quad . \quad . \quad 19$

Figure 3(a): Tree level differential cross section vs. angle . . . . . . 22

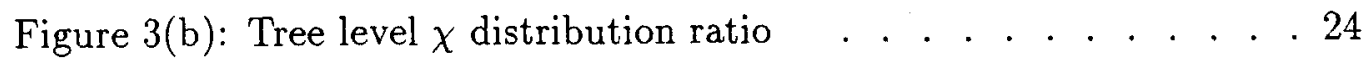

Figure 4: One-loop oblique corrections to $e^{+} e^{-} \rightarrow W^{+} W^{-} \quad \ldots 26$

Figure 5: Notation for vector boson self-energies . . . . . . . 27

Figure 6: Feynman diagrams renormalizing the amplitude

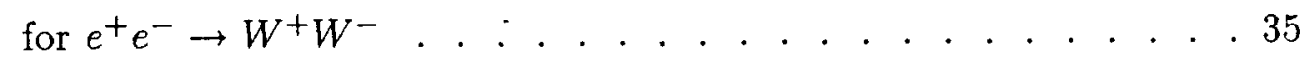

Figure 7: Corrections to the differential cross section for $e^{+} e^{-} \rightarrow W^{+} W^{-}$with various degenerate masses $\ldots . . . .41$

Figure 8: Contribution to fig. 7 from longitudinal polarization only . . 42 
Figure 9: Corrections to the $\chi$ distributions at $\cos \theta=0$,

for degenerate fermions . . . . . . . . . . . . . . . . 43

Figure 10: Corrections to the differential cross section at low energies . 44

Figure 11: Peak structure for split fermion doublets for $e^{+} e^{-} \rightarrow W^{+} W^{-} 45$

Figure 12: Effects of fermions with an isospin-breaking mass splitting . 46

Figure 13: Effects of à supersymmetric generation of scalar partners . 48 
I. INTRODUCTION

$$
-
$$

1 


\section{Loop Expansion}

Quantum field theories are very effective in describing the dynamics of particles. The validity of a model for elementary particle interactions can be tested by comparing experiments with predictions based on the model using quantum field theory. While exact solutions of quantum field theories are very few in number, relativistic perturbation theories are well developed and are quite successful. For theories with small expansion parameters, scattering amplitudes of physical processes can be obtained up to a desired order of accuracy by computing the Feynman diagrams with appropriate numbers of external lines, propagators and loops. Unlike tree diagrams, loop diagrams contain all possible quantum fluctuations of fields that are allowed by the symmetries of a theory. If a theory is renormalizable, one can absorb all divergences of loop diagrams into the parameters of the theory.

The loopwise perturbative expansion, i.e., the expansion according to the increasing number of independent loops of connected Feynman diagrams, may be identified with an expansion in powers of $\hbar$. To see this, consider a loop diagram with $I$-internal lines, $V$-vertices and $L$-independent loops. Due to the topology of the diagrams, the relation $L-1=I-V$ is satisfied. Because the Feynman rules are derived from the functional integral over $\exp \left[i \int \mathcal{L} / \hbar\right]$, the propagator of each quantum field has a factor of $\hbar$ and each vertex has a factor of $\hbar^{-1}$. Thus, the diagram is proportional to $\hbar^{I-V}$ or $\hbar^{L-1}$. Since there is an overall factor of $\hbar^{-1}$ to make the effective action dimensionless, the scattering amplitude is proportional to $\hbar^{L}$. Therefore, the tree level diagrams, the leading order in $\hbar$ describe the classical

limit of the theory. For the case of $L \geq 1$, that is, one or higher loop diagrams, the amplitude depends on $\hbar$ and, hence, are corrections due to quantum mechanical effects. If the loop diagrams correct scattering amplitude through a systematic perturbation theory, higher loops will contain more powers of small coupling constant. Therefore, one loop diagram is the most dominant quantum mechanical contribution to the perturbative computation of a scattering amplitude.

In addition to being the most dominant part of quantum corrections, the one 
loop structure of a theory can also be used to place constraints on the contents of the theory. For example, to guarantee renormalizability, the Ward identities must be preserved at loop levels. Quite generally, if there is a possible difficulty, it arises at the one loop level. Consequently, if there is an anomaly at one loop level, we must restrict the particle contents to cancel the anomaly. This quantum consistency condition, that certain fundamental properties of the theory should be maintained at the quantum level, is an exact theoretical restriction which must be implemented in the theory-from the beginning.

In this thesis, these aspects of the one loop structure of quantum field theories -are investigated in detail by considering two quite different theories. In chapter II, we consider a physical process in the Standard Model in which the one loop diagrams with heavy internal particles give significant quantum corrections. In chapter III, we show how an exact restriction on string models can be obtained from the one loop structure of string dynamics. In this case, the fundamental property that must be implemented in the theory from the beginning is called modular invariance. This consistency condition is quite effective in constraining two-dimensional, generally covariant theories, such as string theories.

As in point particle theories, we can consider the loop diagrams of string theories built from quantum fluctuation of string fields. Even without a complete construction of interacting string field theories, one can still, in principle, compute string scattering amplitudes up to any desired order of the loop expansion. Each order of the loop expansion corresponds to field theory on a two dimensional Riemann surface attached to an appropriate number of external on-shell states. The number of loops corresponds to the genus of the surface in this case. In particular, one loop diagrams correspond to the torus. If we can quantize string field theories canonically, the conclusion that the loop expansion is an expansion in powers of $\hbar$ will hold for string theories, too. However, since the coupling constant is determined dynamically and is not obviously weak, perturbative analyses of string scattering amplitudes may not be valid. 
Nevertheless, by studying two dimensional surfaces with given topology, we may derive a consistency condition that string field theories must satisfy at the quantum level. One such condition is modular invariance. The modular invariance condition arises for the following reason: The set of all two dimensional surfaces with a given topology is parametrized by a set of complex numbers called modular parameters. Such a parametrization is unique up to a set of discrete transformations called modular transformations. Since the loop computation should depend only on the topology of thesurfaces, string scattering amplitudes must be invariant under the modular transformations. The modular transformations are inherent to string theories because they are symmetries of the space of Riemann surfaces. The modular invariance gives strong constraints on the possible string compactifications. In chapter III, we investigate the consequences of imposing the modular invariance on the zero-point one loop scattering amplitudes. 


\section{Unitarity Delay}

Since the $\mathrm{S}$ matrix, by definition, acts on in-coming states to generate out-going states in a scattering process, the unitarity of the $\mathrm{S}$ martrix reflects the fundamental principle of probability conservation. Following the standard formulation of quantum field theories, the unitarity of $\mathrm{S}$ matrix, $S^{\dagger} S=1$ becomes a relation between Green's functions. Writing $S=1+i T$, the unitarity condition becomes a relation between Feynman amplitudes,

$$
2 \operatorname{Im} T_{f i}=\sum_{n} T_{f n} T_{i n}^{*}
$$

For two particle scattering processes, it is very convenient to decompose the amplitudes into partial waves:

$$
T(s, t)=16 \pi \sum_{J}(2 J+1) a_{J}(s) P_{J}(\cos \theta) .
$$

In the process $f \bar{f} \rightarrow V \bar{V}$, where $f$ and $V$ denote a fermion and a vector boson, the partial-wave amplitude $a_{J}$ may be written as

$$
a_{J}=A \frac{s}{M^{2}}+B
$$

where $\mathrm{A}$ and $\mathrm{B}$ are dimensionless constants with some mass scale $M$. For two fermion annihilation processes, only $J=0,1$ states are allowed. If $A \neq 0$ for $a_{1}$, the theory may behave badly at high energies. To preserve the unitarity, tree diagrams must add to make $A=0$. The second constant $B$ need not be cancelled because it does not violate unitarity:

In principle, the restriction $A=0$ may appear only after a full, all-orders calculation. However, if a theory is consistently weakly coupled, we should find $A=0$ (or the stronger restriction $\left|a_{0}\right| \leq 1$ ) at each order of perturbation theory. If these perturbative unitarity bounds are violated, we should conclude that either 
the theory is not unitary, and hence unphysical, or the perturbation theory is not valid. Since $B$ depends only on the parameters of the theory, these perturbative unitarity bounds can also restrict the parameters of the theory. For example, in the standard theory of electroweak interactions, one can give the upper limits on masses of Higgs and heavy fermions using the s-wave unitarity bounds. ${ }^{[1,2]}$

These unitarity bounds become crucial in studying the high-energy limit of theories for the following reason. If the loop corrections increase as a power of the center of mass energy $E$ of the tree level S-matrix element, it is not difficult to see that this theory is not renormalizable. It is argued that in any renormalizable Lagrangian theory, the high-energy unitarity bounds should not be violated in perturbation theory. ${ }^{[3]}$ This leads to the specific requirement of tree unitarity: the $N$-particle S-matrix elements in the tree approximation must grow no more rapidly than $E^{4-N}$ in the limit of high energy $(E)$ at fixed, nonzero angles. If this condition is satisfied at tree level, the loop correction cannot violate unitarity bounds.

While all renormalizable theories satisfy this tree unitarity, in the case of spontaneously broken gauge theories, such as the Standard Model, the unitarity behavior is particularly interesting. In processes like $e^{+} e^{-} \rightarrow W^{+} W^{-}$with longitudinally polarized $W$ 's, or in any process that creates $W_{L}^{ \pm}$and $Z_{L}^{0}$, the S-matrix element can satisfy tree unitarity only when we add correctly both the s-channel and the t-channel tree diagrams. Each of these diagrams contains unitarity violating terms which are exactly cancelled when they are added together. In other words, $A$ in (2.3) vanishes after adding all relevant diagrams. The gauge symmetry plays an important role in gauranteeing the delicate cancellation. This is another statement of the fact that the only renormalizable quantum field theories with massive vector bosons are spontaneously broken gauge theories. Once tree unitarity is realized, unitarity will hold for all orders of perturbation expansion.

However, if we consider one-loop corrections to this process due to heavy particles with mass $M$, since only the s-channel gets the correction, the delicate cancellation between the s- and the t-channel is delayed until energy scale becomes 
comparable with the mass scale $M$. Below $\sqrt{s}=M, A$ gets non-vanishing radiative corrections. Therefore, the tree unitarity is temporarily violated and scattering amplitudes increase linearly with the center of mass energy scale. We refer to this phenomena as unitarity delay.

In chapter II, we will show that we can get a significant enhancement factor like $s / m_{W}^{2}$ in (2.3) for the cross section due to this effect. The magnitude of this enhancement is eventually limited by the unitarity bounds on heavy fermions. The material in chapter If is based on the author's work with Peskin, Lynn, and Selipsky. The results of this analysis have been published previously in ref. 4 . 


\section{Modular Invariance}

The propagation of strings on space-time manifold is described as a two dimensional world sheets, which are Riemann surfaces with a given boundary. If we consider only closed strings, the surfaces are closed with no boundary. String scattering amplitudes can be formulated as functional integrals over fields on these Riemann surfaces. ${ }^{[5]}$ The genus of the surface-corresponds to the number of loops in quantum field theory. In- and out-states of strings are represented by vertex operators which carry definite conformal dimensions and momenta. Since the dynamics of strings does not depend on how we parametrize the world sheet, the -classical action of string theory defined on the two-dimensional surface should have local reparametrization invariance. The action should have another symmetry corresponding to the local Weyl scaling of two-dimensional metric. These two symmetries of the classical, gauge-fixed string action combine to two-dimensional conformal symmetry.

This symmetry is represented by two infinite dimensional chiral algebras for the left- and right-moving sectors of closed strings. These algebras are generated by holomorphic energy-momentum tensor $T(z)$ and anti-holomorphic $\bar{T}(\bar{z})$ and possibly by other generators. The fundamental parameters of these two-dimensional theories are $c$, the strength of the anomaly term in operator product expansion of $T(z),\left(h_{i}, \bar{h}_{i}\right)$ which are the conformal dimensions of primary fields and $C_{i j k}$, the OPE coefficients of the primary fields. The Hilbert space of the theory is represented by $\mathcal{H}=\oplus_{i \mathrm{i}} \mathcal{H}_{\mathrm{i}} \otimes \overline{\mathcal{H}}_{\bar{i}}$ for primary fields $\Phi_{i \bar{i}}(z, \bar{z})$.

The class of theories with conformal symmetry, called conformal field theories, have many interesting features and much applicability to physical systems. ${ }^{[6-9]}$ The main motivation for considering this class of theories is that we can solve these theories exactly in the sense that we can compute any correlation function exactly with the above input parameters by solving a finite number of differential equations derived from Ward identities for the conformal symmetry and from physical arguments. ${ }^{[6]}$ There are two fundamental problems that one would like to solve in 
order to understand conformal field theories. The first one is the classification problem to determine the allowed values of $\left(c, h_{i}\right)$ unambiguously. The second problem is to combine the left-moving and the right-moving sectors together. This sewing problem is very important in solving conformal field thoeries completely. The solution to these problems would give a complete description of string compactifications. The point in this section is that modular invariance is crucial in solving these two problems. In addition to understanding string theories, we can also understand two-dimensional statistical mechanics systems at their criticality using the techniques of conformal field theories. ${ }^{[9]}$

.. We are going to examine the consequences of imposing modular invariance on the torus. We shall do this for several reasons. First, one loop is the simplest and most significant quantum mechanical correction to the scattering amplitudes in perturbation theory. Second, the modular invariance of the torus is relatively simpler than those of higher genus surfaces. Lastly, the modular invariance of the torus is of a fundamental importance in conformal field theories. ${ }^{[10-13]}$ In particular, the further conditions of modular invariance on Riemann surfaces of genus greater than one introduces no additional conditions on the the theory. ${ }^{[14,15]}$

Conformally invariant parameters that enter in specifying the metric of a Riemann surface of a given genus are known as moduli of the Riemann surface, and the space of these parameters is called the moduli space. Some apparently distinct values of different modular parameters may be equivalent, that is, describe same Riemann surface. Therefore, the loop integrals over string world sheets include integrals over the set of points in the moduli space that are not related to each other via modular transformations. The set of all modular transformations form a discrete group, called the modular group. The conformal structure of the torus is uniquely specified by a point $\tau$ in the upper half of complex plane. The modular group is then $S L(2, Z)$, namely the set of all transformations $\tau \rightarrow(a \tau+b) /(c \tau+d)$ wherc $a, b, c, d$ are integers satisfying $a d-b c=1$. The moduli space is the quotient of the upper half plane by $S L(2, Z)$. 
The zero-point scattering amplitude is the simplest object on the torus that one can consider. It is also an important quantity because it can be identified with the partition function of the underlying conformal field theory. The partition function is defined as

$$
Z=\operatorname{Tr} e^{-2 \pi i \operatorname{Rer} P} e^{-2 \pi \operatorname{Im} \tau H}
$$

where the trace is over the Hilbert space, $H=L_{0}+\bar{L}_{0}-c / 12, P=L_{0}-\bar{L}_{0}$, and $\tau$ is the modular parameter. The partition function can be expressed as a sesquilinear form of characters of primary fields for left- and right-moving conformal algebras,

$$
Z=\sum_{h, \bar{h}} \bar{N}_{h, \bar{h}} \chi_{h}(\tau) \bar{\chi}_{\bar{h}}(\bar{\tau})
$$

Each character is defined on a given holomorphic (anti-holomorphic) primary field and its decendents. The partition function in (3.2) shows how the left- and the right-moving sectors should be combined. Only $N_{h, \bar{h}}$ of the primary fields $\Phi_{h, \bar{h}}$ should appear in the spectrum. This is the solution for the sewing problem using the modular invariance. In the examples we will consider, these characters form finite dimensional unitary representations of modular group, ${ }^{[16]}$ and so only subset of the primary fields are allowed in the modular invariant partition functions. Hence we have a selection rule for the primary fields in conformal field theories.

The complete classification problem of conformal field theories is very important, unsolved problem. For $c<1$ conformal field theories, called minimal models, unitarity condition is enough to classify the theories completely. ${ }^{[17]}$ Their modular invariants are also completely classified. ${ }^{[1,19]}$ However, if $c \geq 1$, unitarity is not sufficient. Moreover, imposing modular invariance on the theory results in an infinite number of primary fields. We may in this case introduce new extended chiral symmetries under which the number of primary fields become finite. Only a few classes of extended algebras are known so far. Examples of these symmetries are superconformal ${ }^{[20]}$ parafermionic ${ }^{[21]}$ Kač-Moody symmetries, and 
$W$-algebras. ${ }^{[22]}$ Kač-Moody algebras, which come from the two-dimensional WessZumino-Witten models on group manifolds, ${ }^{[7,8]}$ are particularly interesting because they may give gauge symmetries in low energy string models and because conformal field theories of other classe are given as coset constructions of these algebras. ${ }^{[2,24]}$ Furthermore, many exactly solvable two-dimensional lattice models have been derived from Kać-Moody algebras. ${ }^{[25]}$ Therefore, the classification of Kač-Moody conformal field theories is a very important problem.

There has been significant progress on classification of rational conformal field theories, ${ }^{[10,13,26]}$ which have a finite number of primary fields. Examining the behavior of characters on the torus under $\tau \rightarrow-1 / \tau, \chi_{n}(-1 / \tau)=\sum_{m} S_{n, m} \chi_{m}$, Verlinde, Moore and Seiberg, and others have shown that it might be possible to classify all rational conformal field theories. This implies that all properties at tree level, i.e. on the sphere, and on higher genus can be derived from the modular invariance of the partition functions defined on the torus.

However, finding a complete classification of modular invariant partition functions for theories with extended algebras is still a hard problem. Only theories with the $S U(2)$ Kac-Moody algebra have been completely classified so far. ${ }^{[19]}$ For this theory, the modular invariants are classified by three classes $A, D$ and $E$. Class $A$ consists of diagonal combinations of characters. The characters form a finite unitary representation under the modular group, and hence make the partition function invariant under modular transformations. Class $D$ consists of partition functions of non-simply connected $S O(3)$ group manifolds. Class $E$ consists of the three remaining invariants that do not belong to class $A$ or $D$. Although the complete classification for general group manifolds is a very difficult subject, we construct some partition functions for more general theories using the orbifold approach in chapter III. The material in chapter III is based on work with M. Walton. Parts of this work have been published previously in ref. 27. 
II. DELAYED UNITARITY CANCELLATAION IN $e^{+} e^{-} \rightarrow W^{+} W^{-}$ 


\section{Introduction}

Radiative corrections allow us to probe the high-energy world with comparatively low-energy experiments. Because any intermediate state allowed by symmetry, however heavy, can appear as a quantum fluctuation, precision experiments which isolate radiative corrections can probe for particles with masses much higher than the experimental energy scale. The most sensitive such experiments are those which involve flavor mixing, such as the measurement of the $K_{L}-K_{S}$ mass difference. However, even quantities which entail no special flavor violation, such as the muon ( $\mathrm{g}-2)$, can yield important information on heavy states. Now that we are entering the era of experiments on the properties of the weak vector bosons, it is interesting to think of precision experiments which might be carried out on these new fundamental particles. Such experiments would necessarily be done at energies of $100 \mathrm{GeV}$, or even much higher; still, extending the reach of the available machine energy by measurements sensitive to the radiative corrections is an attractive possibility.

Two important experiments of this type which have been discussed extensively in the literature are the measurements of the $W$ boson mass ${ }^{[28-32]}$ and the polarization asymmetry for fermion pair production at the $Z^{0}$ resonance. $^{[31-33]}$ Both of these experiments are difficult, requiring large statistical samples and methods which cancel systematic errors below the $1 \%$ level. Yet in both cases the influence of new heavy states is larger than one has a right to expect. Naively, one would predict that electroweak radiative corrections due to new particles of mass $M$ would affect the masses and couplings of the weak bosons by terms of order $\alpha / \pi$, times a factor $m_{W}^{2} / M^{2}$ representing the Appelquist-Carazzone decoupling. ${ }^{[34]}$ However, the Appelquist-Carazzone theorem does not apply to theories with chiral gauge couplings or large mass splittings within gauge multiplets, and indeed one finds by explicit calculations both terms with no suppression for $M^{2}>>m_{W}^{2}$ and terms actually enhanced by the factor $\Delta M^{2} / m_{W}^{2}$, with $\Delta M^{2}$ the mass-squared splitting within an isodoublet. ${ }^{[35]}$ The chiral nature of the weak interactions thus 
increases the power of radiative corrections to illuminate new physics.

In this chapter we would like to analyze another set of weak-interaction experiments, to be done at still higher energy. The next step for electron-positron colliders beyond the current generation of $Z^{0}$ resonance machines will be to a linear collider with an energy of order $1 \mathrm{TeV}$ in the center of mass. At such a machine, the most important single process contributing to the electron-positron annihilation cross section is the production of $W$ boson pairs. It is well known that confirmation of the qualitative, tree-level properties of the $W$ pair production cross section already provides a stringent test of the standard model of weak interactions. ${ }^{[36,37]}$ The various diagrams contributing to this process, considered individually, grow faster with $s$ than would be permitted by unitarity. The unitarity constraint on the tree-level amplitude is maintained only by virtue of a delicate cancellation among the various diagrams; this cancellation requires the precise gauge-theory form of the vertices coupling $W$ pairs to the photon and the $Z^{0}{ }^{[3]}$ This observation has been used to propose experimental tests of the idea that $W$ bosons are composite states; indeed, models with composite $W$ bosons produce wildly different cross sections from those of the standard model. ${ }^{[38]}$

We observe here that even within the standard model, the introduction of new heavy particles can cause large deviations from the tree-level cross section. New species with perfectly conventional electroweak couplings naturally yield different radiative corrections to the $s$ and $t$ channel diagrams involved in the tree-level unitarity cancellation. All of these corrections together must sum to zero (to leading order) for asymptotic $s$. However, the regime of greatest experimental interest corresponds to the case of a state with mass $M$ too large to allow its pair-production at the high-energy lepton collider: $s \leq M^{2}$, while $s>m_{W}^{2}$. In this regime, there is no reason for the unitarity cancellations to occur, and, indeed, we find enhanced radiative corrections of order $(\alpha / \pi) \cdot\left(s / m_{W}^{2}\right)$. These effects can be readily identified experimentally. We call this phenomenon, in which heavy-particle radiative corrections postpone the asymptotic cancellation among diagrams, 'unitarity delay'. 
As a part of our calculation, we will give a simplified analysis of the general structure of radiative corrections to $W$ pair production. The radiative corrections due to the conventional states of the standard model have of course been calculated some time ago by Lemoine and Veltman, ${ }^{[39]}$ Philippe, ${ }^{[40]}$ and others. ${ }^{\star}$ However, the structure of the corrections is quite complex, since the theory must be renormalized to the standard model's physical parameters as measured in lower-energy weak interactions. It was observed in ref. 33 that the renormalization program for weak-interaction radiative_corrections at the one-loop level is greatly simplified if one assumes that the virtual particles do not couple directly to light leptons but only to the gauge bosons through their standard-model gauge interactions. This assumption is valid for most new particles one might wish to introduce-heavy quarks, heavy leptons, technicolor bosons, and all of the states of supersymmetric theories except the selectron and the smuon. Lynn, Peskin, and Stuart termed this scheme of coupling 'oblique'. They showed that the oblique radiative corrections to the properties of the $Z$ and $W$ can be represented quite generally by straightforward and manifestly finite expressions. These expressions allow one to classify the various corrections and to understand which precision experiments should give identical and which complementary information on new physics. One of our goals in this chapter is to extend this analysis to the corrections to $e^{+} e^{-} \rightarrow W^{+} W^{-}$.

Accordingly, this chapter will proceed as follows. We begin in Section 2 by reviewing the basic kinematics of $W$ pair production. Following the formalism of Hagiwara, Peccei, Zeppenfeld, and Hikasa, ${ }^{[4]}$ we present formulae for observable differential cross sections in terms of $W$ pair form factors, which might then be analyzed at the one-loop level. In Section 3, we present a general analysis of the oblique weak-interaction radiative corrections to the $W$ form factors. We explicitly extract corrections which are already observable in low-energy and $Z^{0}$ resonance experiments, incorporating these into the effective running electroweak parameters defined by Kennedy and Lynn. ${ }^{[32]}$ What remains is a set of intrinsically

$\star$ See Refs. 41, 42, and 43. An extensive bibliography of theoretical work on the reaction $e^{+} e^{-} \rightarrow W^{+} W^{-}$can be found in Ref. 44. 
new radiative effects; we organize these into manifestly ultraviolet-finite combinations. Finally, we evaluate these new corrections for the case of heavy fermions and scalars. In Section 4 we study the various asymptotic limits of the form factors and confirm the kinematic enhancement of the radiative corrections in the region $s \sim M^{2}>m_{W}^{2}$. We also check explicitly the restoration of the unitarity cancellation for asymptotic $s$. In Section 5 we discuss the physics underlying observability of the corrections, and present numerical examples relevant to future high-energy experiments. We find that a new heavy generation of fermions gives a sizable correction, an enhancement of roughly $0.02 \mathrm{pb}$, constant in $\cos \theta$. At 1 $\mathrm{TeV}$, this represents a $5 \%$ enhancement of the total cross section at non-forward angles. 


\section{General Formalism}

Since our analysis concerns oblique corrections due to new heavy particles, we should expect that the most interesting effects we will uncover will be corrections to the form of the three-gauge-boson vertices. It is easiest to keep track of these corrections by studying the reaction $e^{+} e^{-} \rightarrow W^{+} W^{-}$for vertices of the most general structure, and then inserting the specific expressions for the form factors which arise from explicit one-loop computations. The general analysis which we require has been carried out most efficiently by Hagiwara, Peccei, Zeppenfeld, and Hikasa (HPZH). ${ }^{[4]}$ In this section, we will review their results and express their formulae in a fashion convenient for our analysis.

HPZH begin their analysis with a general parametrization of the WWA and $W W Z$ vertices. In this chapter, we will work in the Euclidean metric. With that convention, their general vertex takes the following form: Let $f_{i}^{V}$ represent form factors $\left(V=A\right.$ or $Z$ ) and $T_{i}$ represent canonical Lorentz structures (implicitly carrying three vector indices). The vertex shown in Fig. 1 is built from these ingredients as

$$
\begin{aligned}
\Gamma_{V}^{\mu \alpha \beta}(q, \bar{q}, P) & \equiv \sum_{i=1}^{7} f_{i}^{V} \cdot T_{i} \\
& =f_{1}^{V} \cdot(q-\bar{q})^{\mu} \delta^{\alpha \beta}+f_{2}^{V} \cdot \frac{(q-\bar{q})^{\mu} P^{\alpha} P^{\beta}}{m_{W}^{2}} \\
& +f_{3}^{V} \cdot\left(P^{\alpha} \delta^{\mu \beta}-P^{\beta} \delta^{\mu \alpha}\right)+f_{4}^{V} \cdot i\left(P^{\alpha} \delta^{\mu \beta}+P^{\beta} \delta^{\mu \alpha}\right) \\
& +f_{5}^{V} \cdot \epsilon^{\mu \alpha \beta \rho}(q-\bar{q})_{\rho}+f_{6}^{V} \cdot i \epsilon^{\mu \alpha \beta \rho} P_{\rho} \\
& +f_{7}^{V} \cdot i \frac{(q-\bar{q})^{\mu} \epsilon^{\alpha \beta \rho \sigma} P_{\rho}(q-\bar{q})_{\sigma}}{m_{W}^{2}}
\end{aligned}
$$

The form factors are dimensionless functions of $s$ and $m_{W}$. We will consistently ignore the electron mass. 


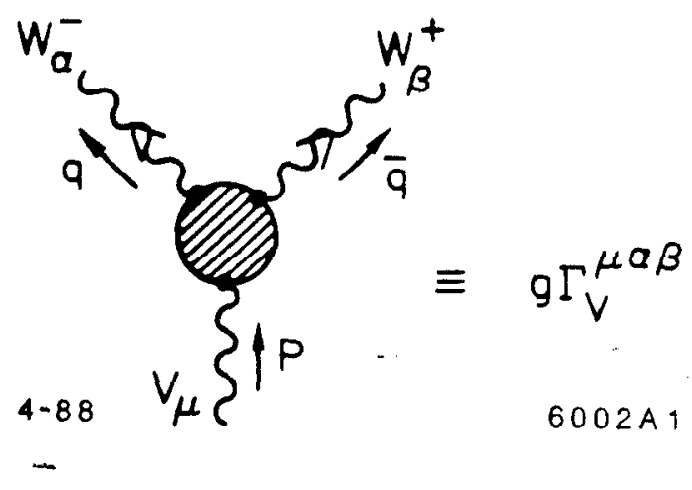

Figure 1. The general vertex for $\mathrm{W}$ pairs.

At the tree level, the $A$ and $Z$ vertices have the same kinematic structure; both are of the form $g_{V} T_{0}$, where

$$
g_{A}=e, \quad g_{Z}=e \cdot \frac{c_{\theta}}{s_{\theta}}
$$

$\left(s_{\theta}\right.$ and $c_{\theta}$ denote $\cos \theta_{w}$ and $\left.\sin \theta_{w}\right)$, and

$$
T_{0}=T_{1}+2 T_{3}=(q-\bar{q})^{\mu} \delta^{\alpha \beta}+2\left(P^{\alpha} \delta^{\mu \beta}-P^{\beta} \delta^{\mu \alpha}\right)
$$

Thus, at the tree level, we would write

$$
f_{1}^{A}=f_{1}^{Z}=1, \quad f_{3}^{A}=f_{3}^{Z}=2,
$$

and set the other form factors to zero.

Using (2.1), we can write the full amplitude arising from the $s$-channel diagrams 


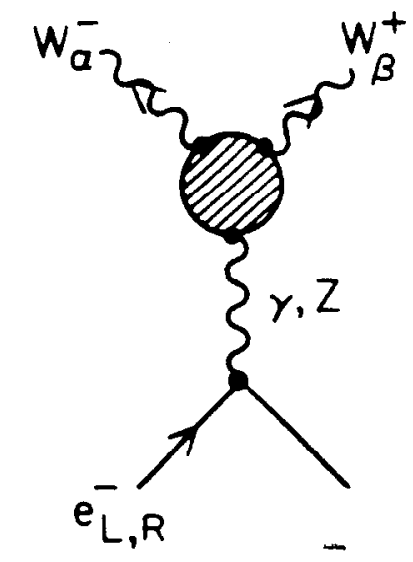

(a)

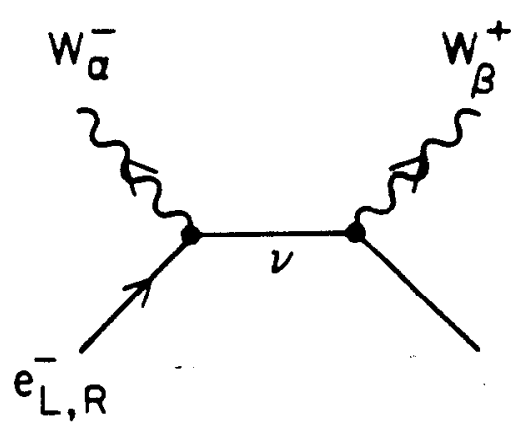

(b)

$6002 A 2$

Figure 2. The amplitude for $e^{+} e^{-} \rightarrow W^{+} W^{-}$: (a) $s$-channel (general vertex); (b) $t$-channel.

for $e^{+} e^{-} \rightarrow W^{+} W^{-}$(Fig. 2(a)) as

$$
\begin{aligned}
\mathcal{M}= & i e^{2} Q\left(\bar{v} \gamma_{\mu} u\right) \frac{1}{P^{2}} \Gamma_{A}^{\mu \alpha \beta} \mathcal{E}_{\alpha}^{*}(q) \mathcal{E}_{\beta}^{*}(\bar{q}) \\
& +i e^{2} \frac{\left(I_{3}-s_{\theta}^{2} Q\right)}{s_{\theta}^{2}}\left(\bar{v} \gamma_{\mu} u\right) \frac{1}{P^{2}+m_{Z}^{2}} \Gamma_{Z}^{\mu \alpha \beta} \mathcal{E}_{\alpha}^{*}(q) \mathcal{E}_{\beta}^{*}(\bar{q})
\end{aligned}
$$

where $P^{2}=-s, u$ and $v$ are electron and positron Dirac spinors, and $\mathcal{E}_{\alpha}(q), \mathcal{E}_{\beta}(\bar{q})$ are polarization vectors of $W^{\mp}$, respectively. We may consider the electron to have definite helicity and write $I_{3}=-\frac{1}{2}$ for $e_{L}, I_{3}=0$ for $e_{R}$. Eq. (2.5) suggests that we combine the photon and $Z$ vertices according to

$$
F_{i}=Q f_{i}^{A}+\frac{\left(I_{3}-s_{\theta}^{2} Q\right)}{s_{\theta}^{2}}\left(\frac{s}{s-m_{Z}^{2}}\right) f_{i}^{Z}, i=1, \cdots, 7
$$

and define $\Gamma^{\mu \alpha \beta}$ as the vertex built from these form factors according to (2.1):

$$
\Gamma^{\mu \alpha \beta}(q, \bar{q}, P)=\sum_{i=1}^{7} F_{i} \cdot T_{i}
$$


Then the matrix element (2.5) can be written more concisely as

$$
\mathcal{M}_{ \pm}=\left(-\frac{i e^{2}}{s}\right) \cdot\left(\bar{v} \gamma_{\mu} u\right) \cdot \Gamma^{\mu \alpha \beta} \cdot \mathcal{E}_{\alpha}^{*}(q) \mathcal{E}_{\beta}^{*}(\bar{q})
$$

The form factors $F_{4}, F_{6}$, and $F_{7}$ multiply $\mathrm{CP}$ violating terms; these always vanish explicitly in the standard model and in the CP-conserving extensions that we will consider here.

It is quite straightforward to evaluate (2.8) directly for each initial and final polarization state by inserting explicit forms for the electron and positron spinors and the $W$ boson polarization vectors. We sketch this development in Appendix A. Following this analysis, we can construct the differential cross section for $W$ scattering from electron and positron states of definite helicity into $W$ states of definite polarization. Expressing these cross sections in units of the point cross section $1 \mathrm{R}=4 \pi \alpha^{2} / 3 s$, we find

$$
\begin{aligned}
\frac{d \sigma}{d \cos \theta} & =\frac{3}{8} \cdot \beta \cdot \Sigma \quad(\mathrm{R}), \\
\Sigma_{T T} & =2 \sin ^{2} \theta\left[\left|A_{1}\right|^{2}-\left(A_{1} A_{2}^{*}+A_{2} A_{1}^{*}\right) \cos \theta+\left|A_{2}\right|^{2}\left(1+2 \cos ^{2} \theta\right)\right] \\
\Sigma_{T L}=\Sigma_{L T} & =\left|A_{3}\right|^{2}\left(1+\cos ^{2} \theta\right)+\left(A_{3} A_{4}^{*}+A_{4} A_{3}^{*}\right) \cos \theta \sin ^{2} \theta+\left|A_{4}\right|^{2} \sin ^{4} \theta \\
\Sigma_{L L} & =\left|A_{5}\right|^{2} \sin ^{2} \theta,
\end{aligned}
$$

where $\theta$ is the scattering angle in the center-of-mass frame, and the subscripts $T, L$ denote transverse or longitudinal polarization of the $W^{-}$and $W^{+}$. For $e_{R}^{-}+e_{L}^{+}$, the $t$-channel diagram does not contribute and so the coefficients $A_{i}$ are built directly from the $F_{i}$ : 


$$
\begin{aligned}
& A_{1}=\beta \cdot F_{1} \\
& A_{2}=0 \\
& A_{3}=\frac{\beta^{2} \sqrt{s}}{m_{W}}\left[\frac{F_{3}}{2}+\frac{\beta \cos \theta}{2} \cdot F_{5}\right] \\
& A_{4}=+\frac{\beta^{2} \sqrt{s}}{2 m_{W}} \cdot F_{5} \\
& A_{5}=\beta \frac{s}{m_{W}^{2}}\left[\frac{F_{3}}{2}-\left(\frac{1}{2}-\frac{m_{W}^{2}}{s}\right) \cdot F_{1}+\frac{\beta^{2}}{4} \frac{s}{m_{W}^{2}} \cdot F_{2}\right]
\end{aligned}
$$

where $\beta$ is the $W$ velocity: $\beta=\left(1-4 m_{W}^{2} / s\right)^{\frac{1}{2}}$. For $e_{L}^{-}+e_{R}^{+}$, we find the more complicated result

$$
\begin{aligned}
& A_{1}=\beta \cdot F_{1}+\frac{\beta}{2 s_{\theta}^{2} \mathcal{D}} \\
& A_{2}=\frac{1}{2 s_{\theta}^{2} \mathcal{D}} \\
& A_{3}=\frac{\beta \sqrt{s}}{m_{W}}\left[\frac{F_{3}}{2}-\frac{\beta \cos \theta}{2} \cdot F_{5}+\frac{1}{2 s_{\theta}^{2}}+\frac{m_{W}^{2}}{s_{\theta}^{2} \beta^{2} s}\left(1-\frac{2 m_{W}^{2}}{s \mathcal{D}}\right)\right] \\
& A_{4}=-\frac{\beta^{2} \sqrt{s}}{2 m_{W}} \cdot F_{5}+\frac{m_{W}}{s_{\theta}^{2} \sqrt{s} \mathcal{D}} \\
& A_{5}=\beta \frac{s}{m_{W}^{2}}\left[\frac{F_{3}}{2}-\left(\frac{1}{2}-\frac{m_{W}^{2}}{s}\right) \cdot F_{1}+\frac{\beta^{2}}{4} \frac{s}{m_{W}^{2}} \cdot F_{2}+\frac{1}{4 s_{\theta}^{2}}\right. \\
& \left.\quad+\frac{1}{\beta^{2} s_{\theta}^{2}} \frac{m_{W}^{2}}{s}\left(1-\frac{2 m_{W}^{2}}{s \mathcal{D}}\right)\right]
\end{aligned}
$$

where $\beta$ is as above and

$$
\mathcal{D}=\frac{1}{2}\left(1+\beta^{2}-2 \beta \cos \theta\right)
$$




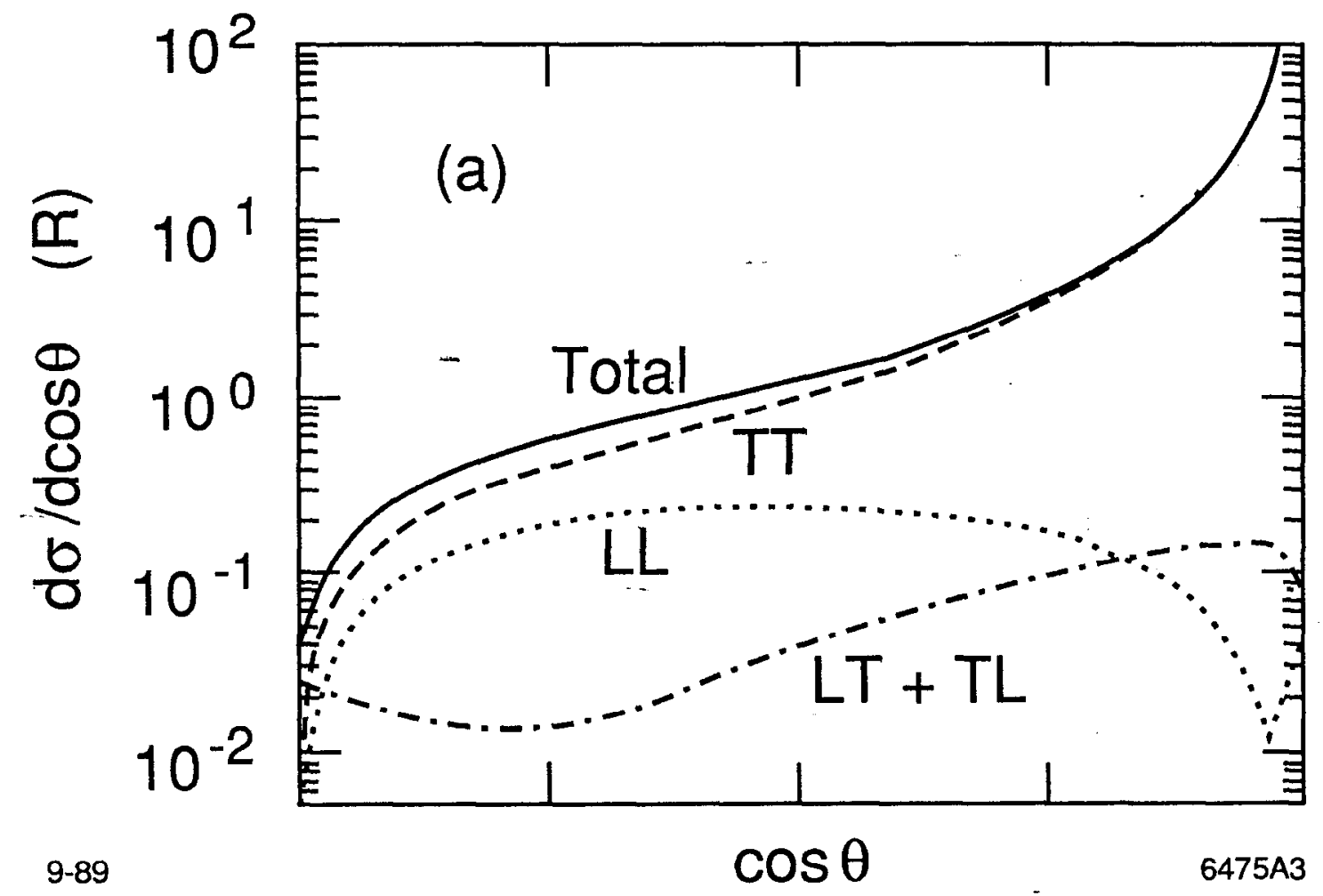

Figure 3(a). Tree level differential cross section vs. $\theta$ : longitudinal polarizations only (LL), mixed polarizations (LT), transverse polarizations (TT), and their sum.

In practice, it is not experimentally straightforward to separate the cross sections for $W$ pair production into the various polarization states. The easiest way to extract some of the information on the $W$ polarization is to use the decay of the $W$ to a charged lepton. The decay distribution obviously depends on whether the $W$ is longitudinally or transversely polarized. Further, the parity violation in the decay amplitude allows one to distinguish the two transverse polarization states. The explicit formula involves only the form factors $A_{i}$ of eqs. (2.10), (2.11). Let $\chi$ be the angle between the $W$ momentum vector and the lepton momentum vector 
as measured in the $W$ rest frame. Then the angular distribution in $\chi$ is given by

$$
\begin{aligned}
& \frac{d \sigma}{d \cos \theta d \cos \chi}\left(e^{+} e^{-} \rightarrow W^{+} \ell^{-} \bar{\nu}\right) \\
& =\frac{9}{32} \cdot \beta \cdot \operatorname{BR}\left(W^{-} \rightarrow \ell^{-} \bar{\nu}\right) \\
& \cdot\left[\Sigma_{T T} \cdot\left(1-\frac{1}{2} \sin ^{2} \chi\right) \pm 4 \cos \theta \sin ^{2} \theta\left|A_{2}\right|^{2} \cdot \cos \chi\right. \\
& \quad+\Sigma_{L T} \cdot\left(1+\frac{1}{2} \sin ^{2} \chi\right) \pm\left(2 \cos \theta\left|A_{3}\right|^{2}+\sin ^{2} \theta\left(A_{3} A_{4}^{*}+A_{4} A_{3}^{*}\right)\right) \cdot \cos \chi \\
& \left.\quad+\Sigma_{L L} \cdot \sin ^{2} \chi\right],
\end{aligned}
$$

where the upper (lower) sign refers to the cross section for $e_{L}^{-} e_{R}^{+}\left(e_{R}^{-} e_{L}^{+}\right)$. The same formula holds for the $\chi$ distributions in $e^{+} e^{-} \rightarrow W^{-} \ell^{+} \nu$ from each electron polarization state. This formula agrees with HPZH; it is a simple byproduct of the analysis leading to (2.9). We discuss its derivation in Appendix A.

The tree-level differential cross sections predicted by eqs. (2.9) and (2.13) are shown in Fig. 3. In Fig. 3(a), we display the differential cross section predicted for $W$ pair production by unpolarized $e^{+} e^{-}$pairs at $\sqrt{s}=1 \mathrm{TeV}$ and the decomposition of the cross section into the contributions from the various $W$ boson polarization states. (In principle, one might also consider the effect of polarizing the elcctrons; however, the contribution from right-handed electrons is generally quite small.) In Fig. 3(b), we plot the $\chi$ distribution at three values of $\cos \theta$. The change in the form of this distribution reflects the increasing proportion of longitudinally polarized $W$ bosons produced as one moves toward the backward direction.

Since the $A_{i}$ are dimensionless scattering amplitudes, they will violate the unitarity limit if they grow asymptotically with any positive power of $s$. For example, eqs. (2.10) and (2.11) show clearly that $A_{5}$ will violate unitarity if the combination of form factors in brackets has asymptotic $s^{0}$ behavior, since this amplitude 


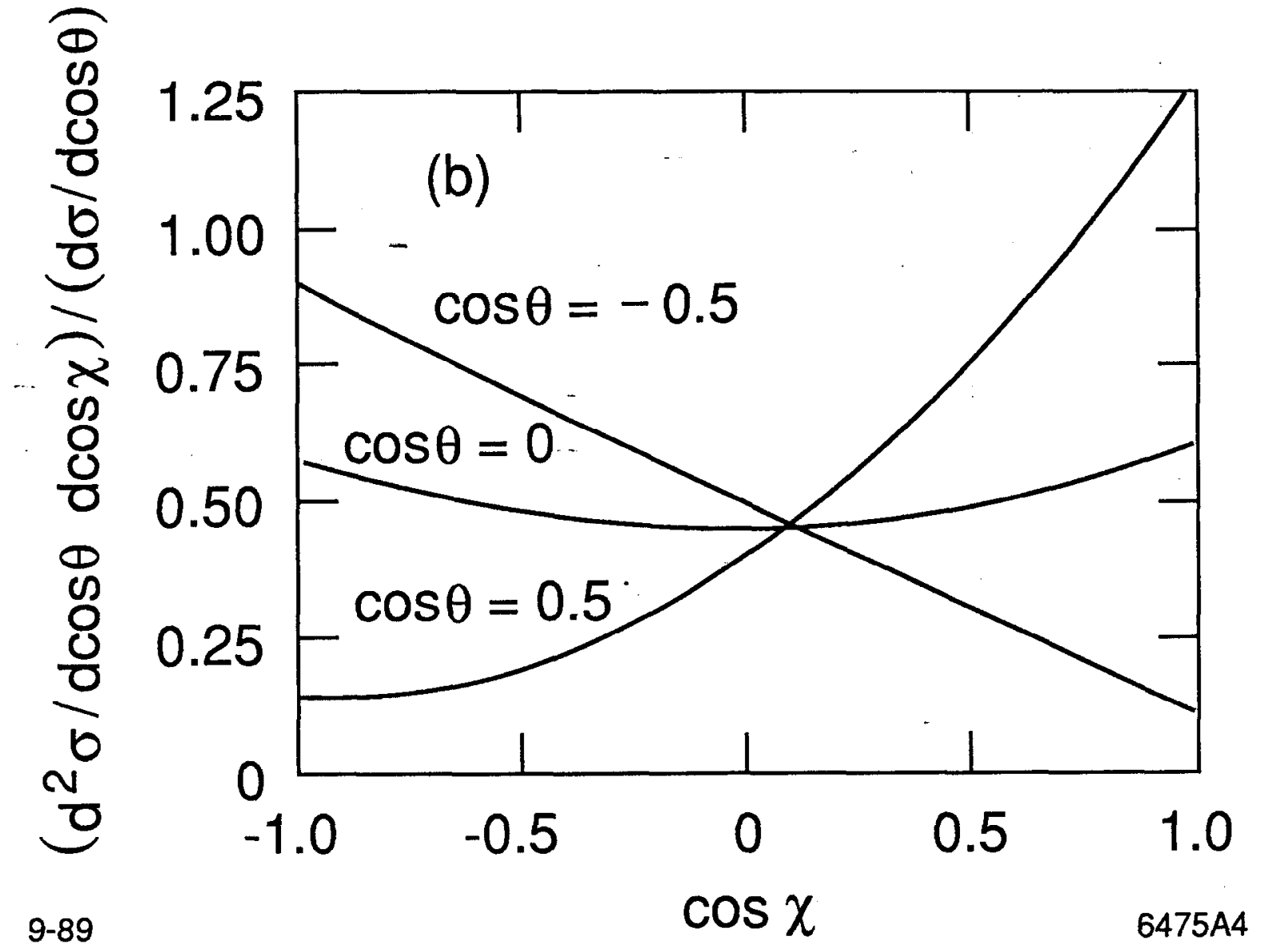

Figure 3(b). Tree level $\chi$ distribution ratio.

contains an overall factor $s / m_{W}^{2}$ arising from the scalar product of longitudinal polarization vectors. At the tree level, (2.4) and (2.6) give

$$
F_{1}=\frac{1}{2} F_{3}=\frac{I_{3}}{s_{\theta}^{2}}\left(1+\frac{m_{Z}^{2}}{s}\right)+\frac{m_{Z}^{2}}{s}+\cdots
$$

Examining (2.10) and (2.11) we see that for right-handed electrons, $I_{3}=0$ and the 
unitarity cancellation is immediate. For left-handed electrons, with $I_{3}=-\frac{1}{2}$, the residual term from the form factors is cancelled by the constant term $1 / 4 s_{\theta}^{2}$, which represents the asymptotic behavior of the $t$-channel diagram.

This type of cancellation should occur order-by-order in perturbation theory. In section 4, we will show this explicitly for one loop radiative corrections due to a heavy generation. The cancellation guarantees good asymptotic behavior up to logarithmic factors. However, the cancellation is guaranteed only for values of $s$ which are actually asymptotic. A new heavy particle of mass $M$ could potentially produce very large radiative corrections by disturbing the delicate cancellations in $A_{5}$ at energies of order $M$ if $M>m_{W}$. In the next section, we will explain how to compute the corrections to the form factors $F_{i}$ which allow us to analyze that situation. 

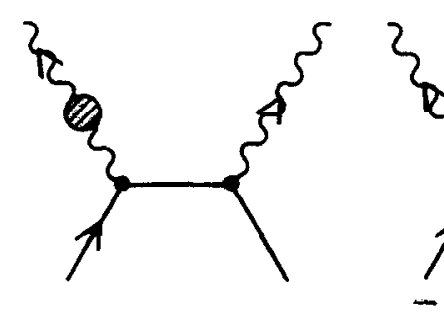

(a)

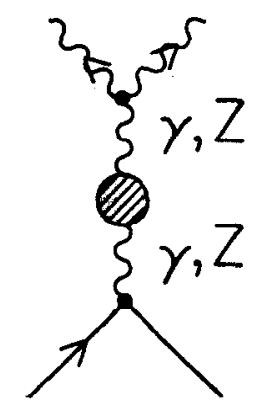

(c)
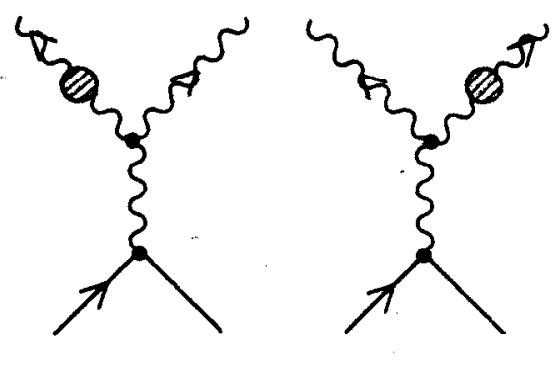

(b)

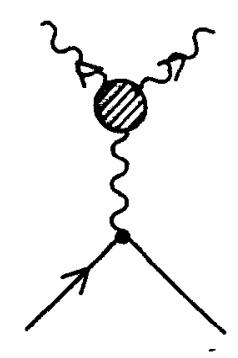

(d)

$6002 A 4$

Figure 4. One-loop oblique corrections to $e^{+} e^{-} \rightarrow W^{+} W^{-}$: (a) corrections to the $t$-channel diagram; (b) external leg corrections to the $s$-channel diagram; (c) propagator corrections to the $s$-channel diagram; (d) vertex corrections to the $s$-channel diagram.

\section{One-Loop Radiative Corrections}

It will be useful to consider the various contributions systematically before beginning an explicit computation of the one-loop corrections. In this chapter we deal only with oblique corrections; this still includes a variety of corrections, as we show in Fig. 4. In the standard model, as long as we have no subdiagrams which involve 


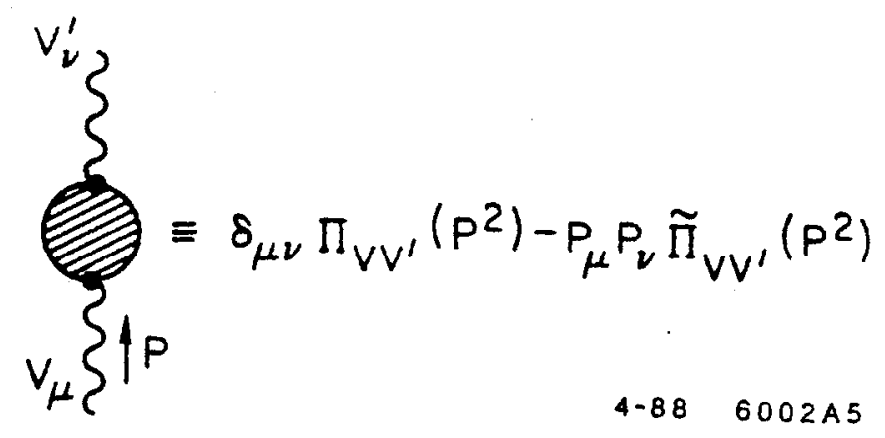

Figure 5. Notation for vector boson self-energies.

Higgs-Higgs or W-W scattering processes (as is the case here), the divergences of all one-loop diagrams are removed when we adjust three basic parameters, which may be taken to be $g, g^{\prime}$ and the Higgs vacuum expectation value or, more concretely, $\alpha, G_{\mu}$, and $m_{Z}$. In this section, we will explain how to renormalize the various diagrams of Fig. 4 and organize them into finite corrections with direct physical meaning.

We would particularly like to address the question of which part of the one-loop corrections to $e^{+} e^{-} \rightarrow W^{+} W^{-}$are already constrained by measurements at low energy or at the $Z^{0}$ and which are new to the $W$ pair production process. To make this separation, we follow Kennedy and Lynn ${ }^{[32]}$ in parameterizing our amplitudes in terms of running electroweak parameters; ref. 32 shows in detail how these quantities summarize the information on weak interaction radiative corrections available from low-energy experiments. From the remaining corrections, we will also extract a finite overall factor representing the $W$ boson wavefunction renormalization. This will leave over other finite contributions which correct the various form factors $f_{i}^{V}$ in the three-gauge-boson vertices. These are the corrections which have the largest physical effect on $W$ pair production.

We begin our analysis by presenting our notation for the loop corrections. These will be given at first in terms of bare parameters (which always carry a subscript 0 ). The boson self energies will be denoted $\Pi_{V V^{\prime}}\left(P^{2}\right)$, as in Fig. 5 . We 
define

$$
\Pi_{V V^{\prime}}^{p} \equiv \frac{\Pi_{V V^{\prime}}}{P^{2}}
$$

The various boson self-energies can be written as two-point functions of the electromagnetic currents $j_{\mathrm{EM}}^{\mu}$ and the weak isospin currents $j_{L}^{\mu i}$ according to

$$
\begin{aligned}
\Pi_{A A} & =e_{0}^{2} \Pi_{Q Q} \\
\Pi_{Z A-} & =\frac{e_{0}^{2}}{s_{0} c_{0}} \cdot\left(\Pi_{3 Q}-s_{0}^{2} \Pi_{Q Q}\right) \\
\Pi_{Z Z} & =\frac{e_{0}^{2}}{s_{0}^{2} c_{0}^{2}} \cdot\left(\Pi_{33}-2 s_{0}^{2} \Pi_{3 Q}+s_{0}^{4} \Pi_{Q Q}\right) \\
\Pi_{W W} & =\frac{e_{0}^{2}}{s_{0}^{2}} \cdot \Pi_{11},
\end{aligned}
$$

where $s_{0}=\sin \theta_{0}$ and $c_{0}=\cos \theta_{0}$ are defined by $s_{0}=e_{0} / g_{0}$. In general, only the rcal parts of these amplitudes are relevant to the $\mathcal{O}(\alpha)$ corrections.

Following ref. 32, we can use Dyson's equations to account for vacuum polarization and boson self energies by exchanging the bare coupling constants for renormalized, running coupling constants (subscripted with a star). This results in an effective Lagrangian with the same form as $\mathcal{L}_{0}$, but with all bare quantities replaced by starred quantities. To include the effects of the oblique corrections we are accounting here, we thus write

$$
\begin{aligned}
\frac{1}{e_{*}^{2}\left(P^{2}\right)} & =\frac{1}{e_{0}^{2}}-\Pi_{Q Q}^{p}\left(P^{2}\right) \\
& =\frac{1}{e_{*}^{2}\left(\mu^{2}\right)}-\left[\Pi_{Q Q}^{p}\left(P^{2}\right)-\Pi_{Q Q}^{p}\left(\mu^{2}\right)\right] \\
\frac{1}{g_{*}^{2}\left(P^{2}\right)} & =\frac{1}{g_{0}^{2}}-\Pi_{3 Q}^{p}\left(P^{2}\right) \\
& =\frac{1}{g_{*}^{2}\left(\mu^{2}\right)}-\left[\Pi_{3 Q}^{p}\left(P^{2}\right)-\Pi_{3 Q}^{p}\left(\mu^{2}\right)\right]
\end{aligned}
$$

from these we define $s_{*}^{2}=e_{*}^{2} / g_{*}^{2}$ and $c_{*}^{2}=1-s_{*}^{2}$. These formulae allow us to relate 
processes occurring at $P^{2}$ to measurements performed at $\mu^{2}$. We similarly define running boson mass parameters to include self-energy and mixing effects:

$$
M_{Z_{*}}^{2}=\frac{e_{*}^{2}}{s_{*}^{2} c_{*}^{2}} \cdot \frac{1}{4 \sqrt{2} G_{\mu *} \rho_{*}}, \quad M_{W_{*}}^{2} \doteq \frac{e_{*}^{2}}{s_{*}^{2}} \cdot \frac{1}{4 \sqrt{2} G_{\mu *}}
$$

with

$$
\begin{gathered}
G_{\mu *}\left(P^{2}\right)=\frac{G_{\mu *}\left(\mu^{2}\right)}{1-\left.4 \sqrt{2} G_{\mu *}\left(\mu^{2}\right)\left(\Pi_{11}-\Pi_{3 Q}\right)\right|_{\mu^{2}} ^{P^{2}}} . \\
- \\
\rho_{*}\left(P^{2}\right)=\frac{1}{1-4 \sqrt{2} G_{\mu *}\left(\Pi_{33}-\Pi_{11}\right)} .
\end{gathered}
$$

-(All starred quantities in this chapter should be evaluated at $P^{2}$, unless explicitly written otherwise). A little algebra yields an explicit form

$$
\begin{aligned}
M_{Z *}^{2}\left(P^{2}\right)=m_{Z}^{2} & +\frac{g_{*}^{2}}{c_{*}^{2}}\left[\left(\Pi_{3 Q}-\Pi_{33}\right)\left(P^{2}\right)-\left(\Pi_{3 Q}-\Pi_{33}\right)\left(-m_{Z}^{2}\right)\right. \\
& +m_{Z}^{2} \cdot\left(c_{*}^{2}-s_{*}^{2}\right)\left(\Pi_{3 Q}^{p}\left(P^{2}\right)-\Pi_{3 Q}^{p}\left(-m_{Z}^{2}\right)\right) \\
& \left.+m_{Z}^{2} \cdot s_{*}^{4}\left(\Pi_{Q Q}^{p}\left(P^{2}\right)-\Pi_{Q Q}^{p}\left(-m_{Z}^{2}\right)\right)\right] .
\end{aligned}
$$

The combination of self-energies on the right-hand side of (3.6) has no uncancelled ultraviolet divergences. With these definitions and light external fermions, the boson propagator and non-Abelian vertex contributions to the neutral-current interactions sum up to the fully renormalized expression ${ }^{[32]}$

$$
\mathcal{M}=\left\{e_{*}^{2} \frac{Q Q^{\prime}}{-s}+\frac{e_{*}^{2}}{s_{*}^{2} c_{*}^{2}} \frac{\left(I_{3}-s_{*}^{2} Q\right)\left(I_{3}^{\prime}-s_{*}^{2} Q^{\prime}\right)}{-s+M_{Z *}^{2}}\right\} .
$$

We use the rcnormalization scheme detailed in ref. 32 :

$$
\begin{aligned}
m_{Z}^{2} & \equiv M_{Z *}^{2}\left(P^{2}=-m_{Z}^{2}\right)=(93.00 \mathrm{GeV})^{2} \\
4 \pi / e_{*}^{2}(0) & =137.036 \\
G_{\mu *}(0) & =1.1581 \times 10^{-5}(\mathrm{GeV})^{-2} .
\end{aligned}
$$


( $G_{\mu}$ differs from $G_{\mu_{*}}(0)$ by residual vertex and box corrections.) All of the divergences in our calculation will be absorbed into the three functions $M_{Z_{*}}^{2}, e_{*}^{2}$, and $g_{*}^{2}$ or $s_{*}^{2}$. Note that in this renormalization scheme, $s_{*}^{2}\left(-m_{Z}^{2}\right)$ is extracted from the measured $m_{Z}$ through terms including $\rho_{*}$. Thus, $s_{*}^{2}\left(-m_{Z}^{2}\right)$ will be affected by large isospin mass splittings.

With this renormalization, contributions from individual fermion generations or scalar doublets are separately gauge invariant and finite; each such contribution can be considered orr its own footing. Accordingly, while our calculations include all electroweak effects of the new heavy particles, they ignore the conventional particles of the standard model, since the standard effects are of order $\alpha$, unenhanced, and smooth as a function of $s$. The standard contributions should of course be included to correctly analyze precision measurements. We also neglect minor corrections from the Higgs and vector boson sector; this eliminates longitudinal self-energy contributions and the need to rediagonalize the $Z$ and photon. ${ }^{[32]}$ Bremsstrahlung effects merely produce an overall multiplicative factor convolved with a hard-photon energy shift, which can be treated ${ }^{(45)}$ straightforwardly and will have no qualitative influence on the effects reported here. Finally, QCD corrections should be quite small at the energies we consider, and we neglect them as well.

In our formulae, the influence of the running of $e_{*}^{2}$ and $s_{*}^{2}$ is relatively minor, and the reader may reproduce the value of any differential cross section that we present to a few percent accuracy by fixing these running parameters at the values

$$
4 \pi / e^{2}=128.0, \quad s_{\theta}^{2}=0.223
$$

$s_{\theta}^{2}$ will be affected by the $\rho$ parameter of course. The $W$ boson mass, unlike the $Z$ mass, appears in our calculation only from the kinematics and should be set directly to its physical value. In the calculations of Section 5, we have used the value of $m_{W}^{2} \equiv M_{W *}^{2}\left(-m_{W}^{2}\right)$ computed from the electroweak theory, including one-loop radiative corrections. This means that we change $m_{W}$ slightly in accord with the properties of the new heavy particles; this change is small except when we 
include heavy generations with very large isospin splitting. Even in the worst case consistent with current $\rho$ parameter measurements $(|\rho-1|<1 \%$, translating to $\left.\Delta m^{2}<(200 \mathrm{GeV})^{2}\right),{ }^{[46,47]}$ one would make an error of less than $2 \%$ in the differential cross section by taking the value $m_{W}=82 \mathrm{GeV}$.

Having defined the parameters of the theory, we can now put together the various corrections to $e^{+} e^{-} \rightarrow W^{+} W^{-}$. We begin with the external leg corrections shown in Fig. 4(a) and (b). These multiply the matrix element by an overall wave function renormalization fäctor

$$
Z_{W}=1+\left.g_{0}^{2} \cdot\left(\frac{\partial}{\partial P^{2}} \Pi_{11}\right)\right|_{P^{2}=-m_{W}^{2}}
$$

For the $t$-channel diagram, this is the only one-loop correction. If we recall that the bare tree diagram is proportional to $g_{0}^{2}$, we can rewrite the overall factor so as to have the same $g_{*}^{2}$ appearing in both channels:

$$
g_{0}^{2} Z_{W}=g_{*}^{2}\left(P^{2}\right) \cdot \frac{g_{0}^{2}}{g_{*}^{2}} Z_{W}=g_{*}^{2}\left(P^{2}\right) \cdot \xi
$$

where

$$
\xi \doteq 1+g_{*}^{2}\left(P^{2}\right)\left(\Pi_{11}^{\prime}\left(-m_{W}^{2}\right)-\Pi_{3 Q}^{p}\left(P^{2}\right)\right)
$$

Since a Ward identity relates vertex and leg corrections, this is a finite object, as may be checked explicitly.

The easiest way to analyze the $s$-channel diagrams is to use the effectiveJagrangian insight in eq. (3.7) that the diagrams of the form $4(\mathrm{c}$ ) simply renormalize the parameters of the zeroth-order diagrams. Folding these corrections into the zeroth-order amplitude, we have

$$
\mathcal{M}=\left(-\frac{i e_{*}^{2}}{s}\right) \cdot\left(\bar{v} \gamma_{\mu} u\right) \cdot\left[Q+\frac{\left(I_{3}-s_{*}^{2} Q\right)}{s_{*}^{2}} \frac{s}{s-M_{Z *}^{2}}\right]\left(T_{0}\right)^{\mu \alpha \beta} \cdot \mathcal{E}_{\alpha}^{*}(q) \mathcal{E}_{\beta}^{*}(\bar{q})
$$

where $T_{0}$ is the tensor (2.3). We then consider the diagrams of Fig. 4(b) to multiply 
this amplitude by the additional and divergent factor

$$
Z_{W}=\xi \cdot\left(1+g_{*}^{2} \Pi_{3 Q}^{p}\left(P^{2}\right)\right)
$$

Finally, we must include the true vertex corrections shown in Fig. 4(d). In order to keep track of the electroweak currents as in (3.1), we notate these corrections as

$$
\Gamma_{A}^{\mu \alpha \beta} \equiv e_{*} g_{*}^{2} \cdot \Sigma_{Q+-}^{\mu \alpha \beta} \quad, \quad \Gamma_{Z}^{\mu \alpha \beta} \equiv \frac{e_{*}}{s_{*} c_{*}} g_{*}^{2} \cdot\left(\Sigma_{3+-}^{\mu \alpha \beta}-s_{*}^{2} \cdot \Sigma_{Q+-}^{\mu \alpha \beta}\right)
$$

using $g_{0}^{2}=g_{*}^{2}$ to the required accuracy. Then the diagrams of Fig. 4(d) yield an additional term

$$
\begin{aligned}
& \mathcal{M}=\left(-\frac{i e_{*}^{2}}{s}\right) \cdot\left(\bar{v} \gamma_{\mu} u\right) \\
& \times\left[Q \Sigma_{Q+-}^{\mu \alpha \beta}+\frac{\left(I_{3}-s_{*}^{2} Q\right)}{s_{*}^{2} c_{*}^{2}} \cdot \frac{s}{\left(s-m_{Z}^{2}\right)}\left(\Sigma_{3+-}^{\mu \alpha \beta}-s_{*}^{2} \Sigma_{Q+-}^{\mu \alpha \beta}\right)\right] \cdot \mathcal{E}_{\alpha}^{*}(q) \mathcal{E}_{\beta}^{*}(\bar{q})
\end{aligned}
$$

Here we can neglect the $\mathcal{O}\left(g^{2}\right)$ difference between $M_{Z *}^{2}$ and $m_{Z}^{2}$, although in (3.13) we must retain corrections proportional to $M_{Z^{*}}^{2}-m_{Z}^{2}$. There it is useful to expand the denominator $\left(s-M_{Z^{*}}^{2}\right)$ to first order about $\left(s-m_{Z}^{2}\right)$; then the zeroth-order term can enter the tree-level unitarity cancellation unchanged. The results of eqs. (3.13), (3.14), and (3.16) can thus be combined to form the following expression for the sum of the $s$-channel diagrams: 


$$
\begin{aligned}
\mathcal{M}=\left(-\frac{i e_{*}^{2}}{s}\right) & \cdot \xi \cdot\left(\bar{v} \gamma_{\mu} u\right) \cdot\left\{\left[Q+\frac{\left(I_{3}-s_{*}^{2} Q\right)}{s_{*}^{2}} \frac{s}{s-m_{Z}^{2}}\right] \cdot T_{0}^{\mu \alpha \beta}\right. \\
& +\frac{\left(I_{3}-s_{*}^{2} Q\right)}{s_{*}^{2}} \frac{s}{s-m_{Z}^{2}} \frac{M_{Z *}^{2}-m_{Z}^{2}}{s-m_{Z}^{2}} \cdot T_{0}^{\mu \alpha \beta} \\
& +\left[Q \cdot g_{*}^{2}\left(\Sigma_{Q+-}^{\mu \alpha \beta}+\Pi_{3 Q}^{p} T_{0}^{\mu \alpha \beta}\right)\right. \\
& \left.\left.+\frac{\left(I_{3}-s_{*}^{2} Q\right)}{s_{*}^{2} c_{*}^{2}} \frac{s}{s-m_{Z}^{2}} g_{*}^{2} \cdot\left(\Sigma_{3+-}^{\mu \alpha \beta}-s_{*}^{2} \Sigma_{Q+-}^{\mu \alpha \beta}+c_{*}^{2} \Pi_{3 Q}^{p} T_{0}^{\mu \alpha \beta}\right)\right]\right\} \\
& \cdot \mathcal{E}_{\alpha}^{*}(q) \mathcal{E}_{\beta}^{*}(\bar{q}) .
\end{aligned}
$$

Each line of (3.17) has cancelling ultraviolet divergences, since $\Sigma_{Q+-}, \Sigma_{3+-}$, and $-\Pi_{3 Q}^{p} T_{0}$ contain identical divergences. In the first line, we have separated out a piece proportional to the zeroth order $s$-channel amplitude; when this is added to the $t$-channel amplitude, the sum is simply the zeroth-order amplitude evaluated with running coupling constants and multiplied by $\xi$. The remaining three lines of (3.17) give intrinsically new corrections.

We expect that the full one-loop-corrected amplitude should obey perturbative unitarity. In the combination of the $t$-channel amplitude with the first line of (3.17), the unitarity cancellation is explicit; eqn. (3.11) arranges for both channels to have $g_{*}^{2}\left(P^{2}\right)$ as the coupling and $\xi$ as an overall factor. For the remaining terms in (3.17), we can only check case by case that the leading, unitarity-violating $s$ dependence cancels when $s$ is large. If the loop diagrams contain a heavy species of mass $M$, we cannot expect this cancellation to occur except when $s>>M^{2}$. Thus, when $s \sim M^{2}>>m_{W}^{2}$, we expect the last three lines of (3.17) to produce radiative corrections enhanced by a factor $\left(s / m_{W}^{2}\right)$. These are the dominant effects arising from our analysis.

We conclude this section by converting the amplitude (3.17) into a set of form factors which can be inserted into the formulae of Section 2 . If we use $T_{0}=T_{1}+2 T_{3}$ 
and decompose each vertex function according to

$$
\Sigma^{\mu \alpha \beta} \equiv \sum_{i=1}^{7} T_{i} \cdot \Sigma^{(i)}
$$

we can read from (3.17)

$$
\begin{aligned}
& f_{1}^{A}=1+g_{*}^{2} \cdot\left[\Sigma_{Q}^{(1)}+\Pi_{3 Q}^{p}\right] \\
& f_{1}^{Z}=1+\frac{g_{*}^{2}}{c_{*}^{2}} \cdot\left[\left(\Sigma_{3}^{(1)}-s_{*}^{2} \Sigma_{Q}^{(1)}\right)+c_{*}^{2} \Pi_{3 Q}^{p}\right]+\frac{M_{Z *}^{2}-m_{Z}^{2}}{s-m_{Z}^{2}} \\
& f_{3}^{A}=2+g_{*}^{2} \cdot\left[\Sigma_{Q}^{(3)}+2 \Pi_{3 Q}^{p}\right] \\
& f_{3}^{Z}=2+\frac{g_{*}^{2}}{c_{*}^{2}} \cdot\left[\left(\Sigma_{3}^{(3)}-s_{*}^{2} \Sigma_{Q}^{(3)}\right)+2 c_{*}^{2} \Pi_{3 Q}^{p}\right]+2 \frac{M_{Z *}^{2}-m_{Z}^{2}}{s-m_{Z}^{2}} \\
& f_{i}^{A}=g_{*}^{2} \cdot\left[\Sigma_{Q}^{(i)}\right] \\
& f_{i}^{Z}=\frac{g_{*}^{2}}{c_{*}^{2}} \cdot\left[\left(\Sigma_{3}^{(i)}-s_{*}^{2} \Sigma_{Q}^{(i)}\right)\right] \quad i=2,5
\end{aligned}
$$

To use these form factors, we must also make two modifications in the formulae of section 2: first, the coupling constants $e^{2}, s_{\theta}^{2}$ should be replaced by $e_{*}^{2}, s_{*}^{2}$; second, the final cross sections should be multiplied by the factor $|\xi|^{2}$ defined in eq. (3.12). Both of these corrections are numerically quite small, although one should note that, for light fermions or scalars, $\xi$ contains logarithmic factors which are important in the correct coupling constant evolution of the threc-gauge-boson vertex. 

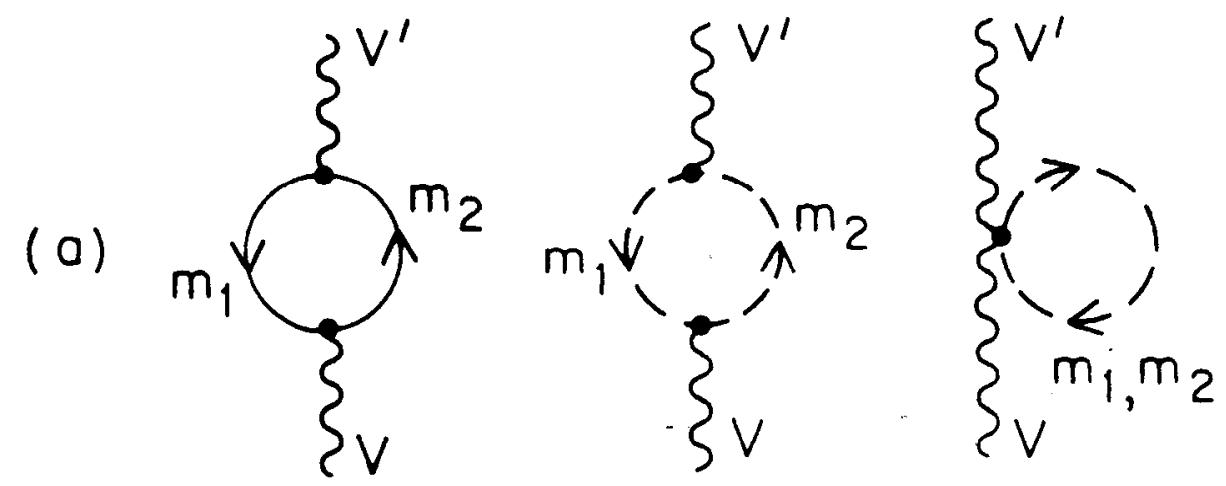

(b)
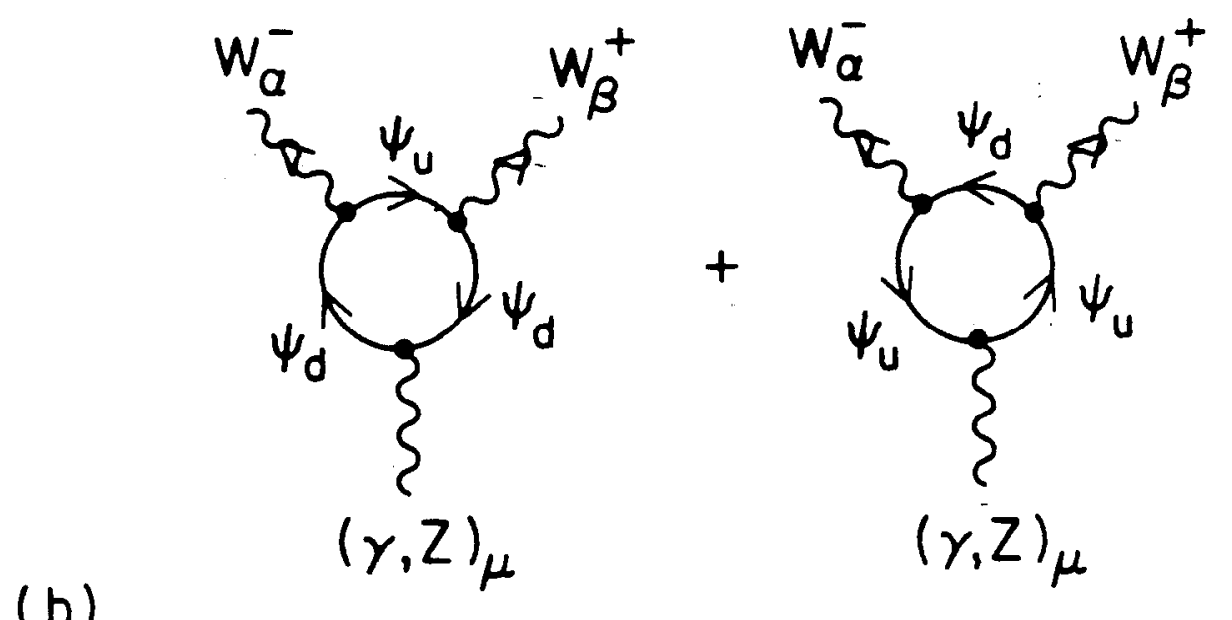

\section{4-88}
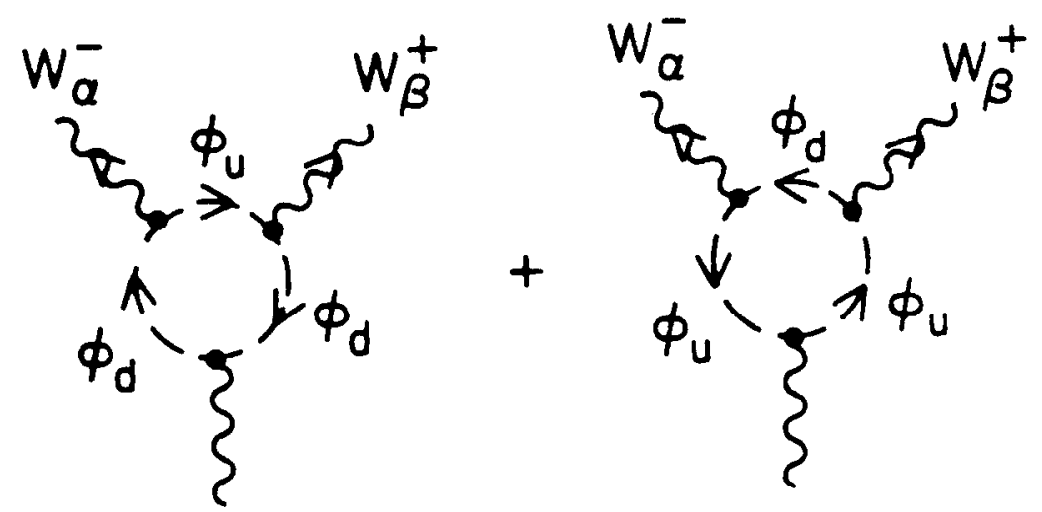

$6002 A 6$ $(\gamma, Z)_{\mu}$

Figure 6. Feynman diagrams renormalizing the amplitude for $e^{+} e^{-} \rightarrow W^{+} W^{-}$:

(a) two-point functions; (b) three-point functions. 


\section{Low and High Energy Behavior}

We are now in a position to evaluate the various Feynman diagrams contributing one loop corrections to the process $e^{+} e^{-} \rightarrow W^{+} W^{-}$and to organize the results explicitly into finite corrections. For heavy fermions, we consider the diagrams shown in Fig. 6(a), (b). The actual formulae for the various corrections are complicated and, in themselves, rather unilluminating, so we have chosen to display these expressions only in Appendix B. In this section and the next, we will discuss their important properties. Here, we analyze the formulae analytically in the limits of high and low energy. For a heavy generation of mass $M$ we will show explicitly the presence of enhanced radiative corrections when $s<<M^{2}$ and also a perturbative unitarity cancellation in the radiative corrections for $s>>M^{2}$. In section 5 we will study the formulae numerically for general values of $s$.

In our presentation of the complete results given in Appendix B, we have followed the method of Passarino and Veltman ${ }^{[18]}$ in expressing the various diagrams in terms of a fixed set of standard one-loop integrals. One can then evaluate these

integrals analytically; ${ }^{[49]}$ tailored computer programs exist for this purpose. ${ }^{[0,51]}$ In our analysis, we have found it convenient to make some further simplifications, including the explicit cancellation of ultraviolet divergences, and to write our results in terms of a set of finite and dimensionless reduced Passarino-Veltman functions. These functions are defined, and their asymptotic forms are presented, in Appendix C. The results of this section can then be obtained by inserting the appropriate asymptotic formulae into the results for the form factors given in Appendix B.

\subsection{Nondecoupling Effects at Low Energy}

We consider first the case of radiative corrections for $s$ well below the heavy fermion threshold. As we have explained, we expect in this region to find terms enhanced by a factor $\left(s / m_{W}^{2}\right)$. Ordinarily, one might expect that loop corrections due to heavy fermions are suppressed by powers of $\left(s / M^{2}\right)$ because of AppelquistCarazzone decoupling. However, with chiral currents or large doublet mass split- 
tings, it is possible to evade the decoupling theorem and isolate a finite contribution. Clearly, degenerate scalar particles will not exhibit this effect, as we discuss in section 5 .

Let us then expand the expressions of Appendix B, for $s$ in the range $m_{W}^{2} \ll$ $s \ll M^{2}$, assuming a fermion doublet with hypercharge $Y$ and masses $m_{u}$ and $m_{d}$. Defining

$$
\Delta m^{2}=m_{\underline{u}}^{2}-m_{d}^{2} \quad \text { and } \quad m^{2}=\frac{1}{2}\left(m_{u}^{2}+m_{d}^{2}\right),
$$

with $\Delta m^{2} \ll m^{2}$, we find

$$
\begin{aligned}
& F_{1} \cong \frac{\alpha}{4 \pi s_{\theta}^{2}}\left[-Y \cdot \frac{1}{3 c_{\theta}^{2}} \cdot \frac{\Delta m^{2}}{m^{2}}+\frac{I_{3}}{s_{\theta}^{2} c_{\theta}^{2}}\left(-\frac{c_{\theta}^{2}}{6}-Y \cdot \frac{s_{\theta}^{2}}{3} \cdot \frac{\Delta m^{2}}{m^{2}}\right)\right] \\
& F_{2} \cong 0 \\
& F_{3} \cong \frac{\alpha}{4 \pi s_{\theta}^{2}}\left[-Y \cdot \frac{7}{12 c_{\theta}^{2}} \cdot \frac{\Delta m^{2}}{m^{2}}+\frac{I_{3}}{s_{\theta}^{2} c_{\theta}^{2}}\left(-\frac{c_{\theta}^{2}}{3}-Y \cdot \frac{7 s_{\theta}^{2}}{12} \cdot \frac{\Delta m^{2}}{m^{2}}\right)\right] \\
& F_{5} \cong \frac{\alpha}{4 \pi s_{\theta}^{2}}\left[-\frac{1}{24 c_{\theta}^{2}} \cdot \frac{\Delta m^{2}}{m^{2}}-Y \cdot \frac{1}{3}\left(1+\frac{1}{c_{\theta}^{2}}\right)+\frac{I_{3}}{s_{\theta}^{2} c_{\theta}^{2}}\left(-\frac{1}{3} \cdot Y-\frac{1}{24} \cdot \frac{\Delta m^{2}}{m^{2}}\right)\right]
\end{aligned}
$$

where $I_{3}=-\frac{1}{2}, 0$ for $e_{L}^{-}, e_{R}^{-}$. These formulae simplify dramatically if we include a full generation in which all the doublets have the same masses, namely, $\left[\frac{\Delta m^{2}}{m^{2}}\right]_{\text {lepton }}=\left[\frac{\Delta m^{2}}{m^{2}}\right]_{\text {quark }}$, and use the fact $\sum_{\text {doublets }} Y=0$;

$$
\begin{aligned}
& F_{1} \cong \frac{\alpha}{4 \pi s_{\theta}^{2}}\left(-\frac{2 I_{3}}{3 s_{\theta}^{2}}\right) \\
& F_{2} \cong 0 \\
& F_{3} \cong \frac{\alpha}{4 \pi s_{\theta}^{2}}\left(-\frac{4 I_{3}}{3 s_{\theta}^{2}}\right) \\
& F_{5} \cong \frac{\alpha}{4 \pi s_{\theta}^{2}}\left(\frac{I_{3}}{s_{\theta}^{2}}+1\right)\left(-\frac{1}{6 c_{\theta}^{2}} \frac{\Delta m^{2}}{m^{2}}\right) .
\end{aligned}
$$

Note that only $F_{5}$ depends on the mass splitting and $F_{1}, F_{2}$ and $F_{3}$ are zero for 
the right-handed electron. For left-handed electrons, the process $e^{+} e_{L}^{-} \rightarrow W_{L}^{+} W_{L}^{-}$ will show leading behavior

$$
A_{5}(1-\text { loop }) \cong \frac{s}{m_{W}^{2}} \cdot \frac{\alpha}{4 \pi s_{\theta}^{2}} \cdot \frac{1}{6 s_{\theta}^{2}}
$$

and the cross section for $e^{+} e_{L}^{-} \rightarrow W_{L}^{+} W_{L}^{-}$becomes

$$
\frac{d \sigma}{d \Omega} \cong \frac{d \sigma}{d \Omega}(\text { tree }) \cdot\left[1+2 \cdot \frac{A_{5}(1-\text { loop })}{A_{5}(\text { tree })}\right]
$$

where $A_{5}$ (tree) is given by $(2.11)$; thus

$$
\frac{\delta\left(\frac{d \sigma}{d \Omega}\right)}{\left(\frac{d \sigma}{d \Omega}\right)} \cong\left(\frac{\alpha c_{\theta}^{2}}{3 \pi s_{\theta}^{2}}\right) \cdot \frac{-s}{m_{W}^{2}} \cong\left(2.9 \times 10^{-3}\right) \cdot \frac{s}{m_{W}^{2}}
$$

This radiative correction is proportional to the number of heavy generations; aside from the effects of isospin mass splittings on the $\rho$ parameter, it does not depend on the masses of the heavy generation as long as $s \ll m^{2}$ and lepton/quark mass differences are small. The factor $10^{-3}$ is typical of one loop radiative corrections, but the enhancement factor $s / m_{W}^{2}$ yields a $10 \%$ effect for $\sqrt{s}=500 \mathrm{GeV}$. This relative enhancement continues rising, quadratically in energy, until it is cut off above threshold. In essence, the unitarity delay effect can be thought of as adding a constant 0.02 pbarn to a tree-level cross section which is falling like $1 / \mathrm{s}$. The unitarity delay thus exists and is measurable at lower energies, but it would be advantageous to use as high an energy as possible.

\subsection{Asymptotic Behavior at High Energy}

We now consider the case $s \gg m^{2} \gg m_{W}^{2}$, including one heavy generation where all fermions are of equal mass $m$. As already mentioned in section 2 , any uncancelled leading $s^{0}$ behavior in the form factors $F_{i}$ will violate unitarity because of the factor $\frac{s}{m_{W}^{2}}$ in $A_{5}$. We check this cancellation below, keeping next-to-leading order terms as a check on our numerical results and to provide physical insight into the system's high-energy behavior. 
Referring to the appendices, the $F_{i}$ can be seen to tend asymptotically to

$$
\begin{aligned}
F_{1} & \cong-\frac{\alpha}{4 \pi s_{\theta}^{2}} \cdot \frac{2}{3} \frac{I_{3}}{s_{\theta}^{2}} \\
\frac{s}{m_{W}^{2}} \cdot F_{2} & \cong-\frac{\alpha}{4 \pi s_{\theta}^{2}}\left[\frac{4}{3} \frac{I_{3}}{s_{\theta}^{2}}+\frac{m^{2}}{s} \frac{I_{3}}{s_{\theta}^{2}}\left(32-16 \ln \frac{s}{m^{2}}+4 \ln ^{2} \frac{s}{m^{2}}-4 \pi^{2}\right)\right] \\
F_{3} & \cong-\frac{\alpha}{4 \pi s_{\theta}^{2}} \frac{m^{2}}{s}\left[\frac{4}{c_{\theta}^{2}}\left(1+\frac{I_{3}}{s_{\theta}^{2}}\right)\left[\ln \frac{s}{m^{2}}-2\right]-2 \frac{I_{3}}{s_{\theta}^{2}}\left[\ln ^{2} \frac{s}{m^{2}}-\pi^{2}\right]\right] \\
F_{5} & \cong 0
\end{aligned}
$$

thus

$$
A_{5}(1-\text { loop }) \cong-\frac{\alpha}{4 \pi s_{\theta}^{2}} \cdot \frac{m^{2}}{m_{W}^{2}} \cdot\left(\frac{I_{3}}{s_{\theta}^{2}}\left(\frac{2}{c_{\theta}^{2}}-4\right)+\frac{2}{c_{\theta}^{2}}\right) \cdot\left[\ln \frac{s}{m^{2}}-2\right]
$$

Notice that the leading $s^{0}$ terms in the $F_{i}$ are cancelled in $A_{5}$, a result of unitarity cancellation at the one loop level. Also cancelled are all dilogarithms. Even so, if $m^{2} \gg m_{W}^{2}$ then the magnitude of $A_{5}\left(1\right.$-loop) can be as large as that of $A_{5}$ (tree) in (2.10) and (2.11). The perturbative expansion requires careful examination at high energy with a sufficiently heavy fermion generation, as we shall discuss in detail in section 5 . 


\section{Numerical Results and Discussion}

We can now compare the above results with numerical calculations and discuss the experimental observability of the heavy particle corrections. In assessing the size of these corrections, one should remember that nondecoupling effects generally arise from the breaking of global symmetries in association with large dimensionless parameters. For heavy fermions in the standard model, these parameters might arise either from isospin-breaking mass differences or from the large Yukawa couplings needed to generate even large isospin-symmetric massses. We should assess the relative importance of these two contributions. For scalars, only the isospin -splitting of masses arises from a symmetry breaking, and so here there is only one possible source for the effect.

Let us begin with the case of a heavy, isospin-degenerate fermions. The detailed forms of the radiative corrections to the $W$ form factors, valid over the full range of energies, are presented in Appendix B. By inserting these expressions into (2.9), we obtain the effects of the heavy fermions on the differential cross section for $W$ pair-production. In Fig. 7, we plot the corrected differential cross section at $\cos \theta=0$, incorporating effects of a degenerate heavy generation of fermions, for several different masses. (Integration over $\cos \theta$ merely shifts the whole curve upward by including the unenhanced forward peak). We can see that the radiative correction gives a small but noticeable effect at low energies and contributes a significant enhancement of the cross section in a region within a factor of 2 in

$\sqrt{s}$ of the pair production threshold. The suggestion from the analytic formulae of an effect increasing quadratically with energy is actually well confirmed by the numerical results shown in Fig. 7. Note the rapid onset of unitarity cancellations above threshold.

The physics of the correction terms is clarified by a more detailed look at the numerical results. Since the delayed unitarity cancellation affects mainly the cross section for producing pairs of longitudinal $W$ bosons, we should expect that the enhanced radiative corrections appear mainly in that polarization state. Indeed, 


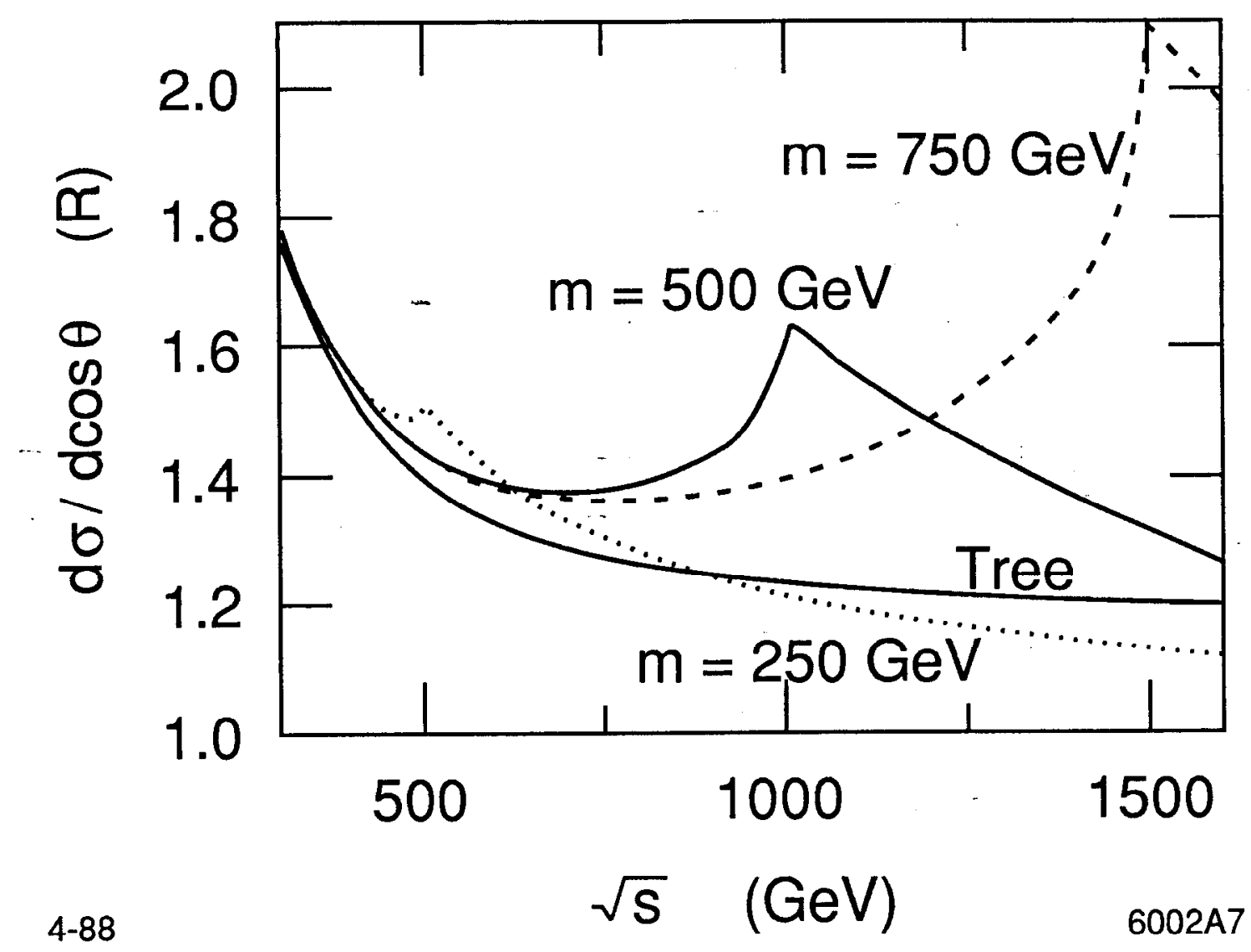

Figure 7. Corrections to the differential cross section for $e^{+} e^{-} \rightarrow W^{+} W^{-}$with various degenerate fermion masses, at $\cos \theta=0$.

Fig. 8 shows the contributions to the cross sections of Fig. 7 from longitudinally polarized $W$ pairs; the enhancement of this polarization state is very large and accounts for essentially the whole effect. The heavy fermions make at most a $2 \%$ correction to the cross section due to the other polarization states. The importance of the longitudinal $W$ pairs can be assessed in another way, which can be observed directly in experiments: in Fig. 9, we plot the distribution of the lepton decay angle $\cos \chi$ in the presence of heavy fermion corrections. The enhancement near 


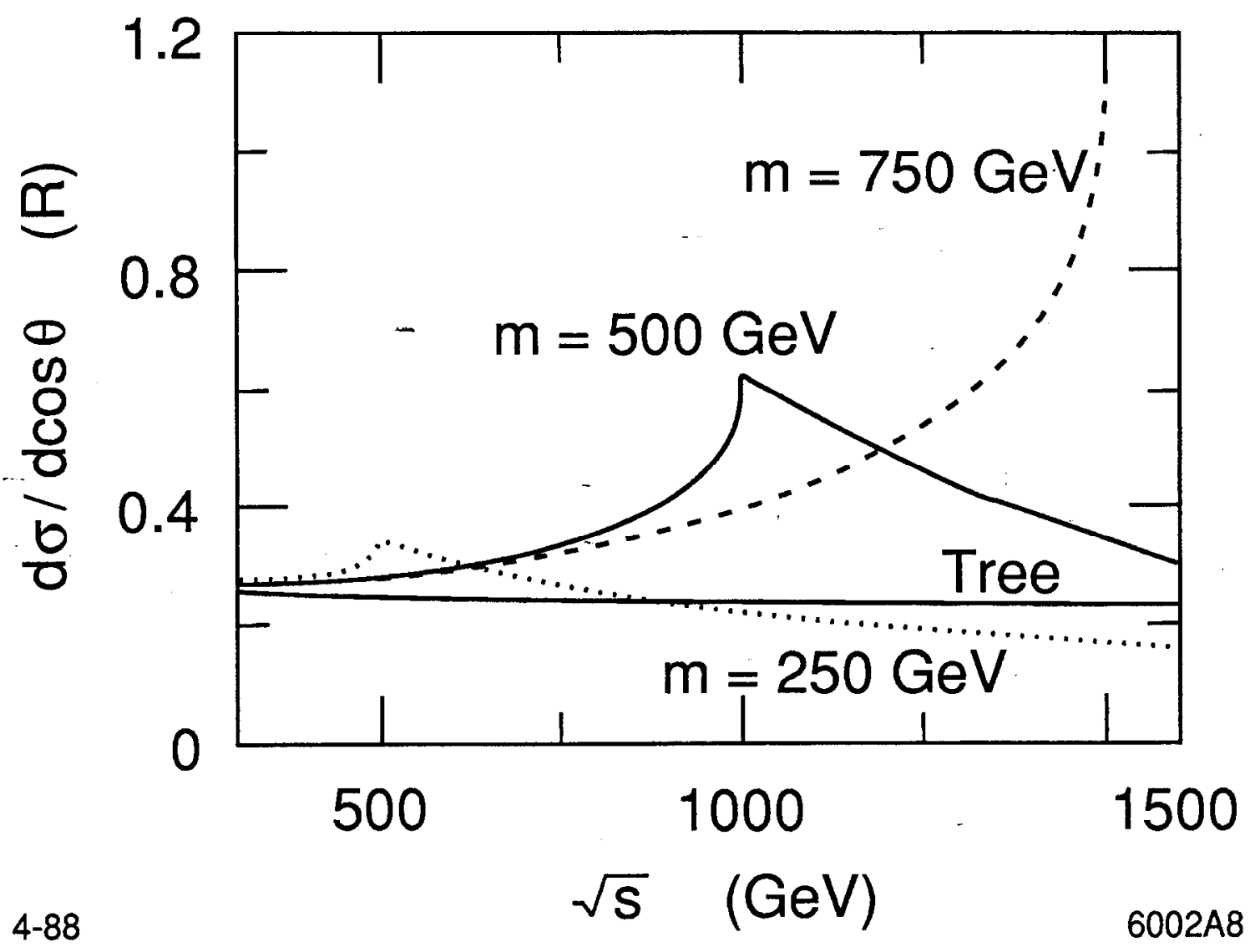

Figure 8. Contribution to fig. 7 from longitudinal polarizations only, at $\cos \theta=0$.

$\cos \chi=0$ indicates the increasing importance of longitudinally polarized $W$ bosons. The dependence on $\cos \theta$ of the heavy fermion corrections shows less structure; the corrections are roughly independent of $\cos \theta$. However, for $\cos \theta>0.5$, the $W$ pair production cross section is dominated by transversely polarized pairs, and the relative enhancement due to radiative corrections disappears.

Eq. (4.6) displays the low energy limit of the correction term. Well below threshold, this contribution is independent of the heavy fermion masses. We confirm this result in Fig. 10 by plotting the differential cross section at $\cos \theta=0$ for 


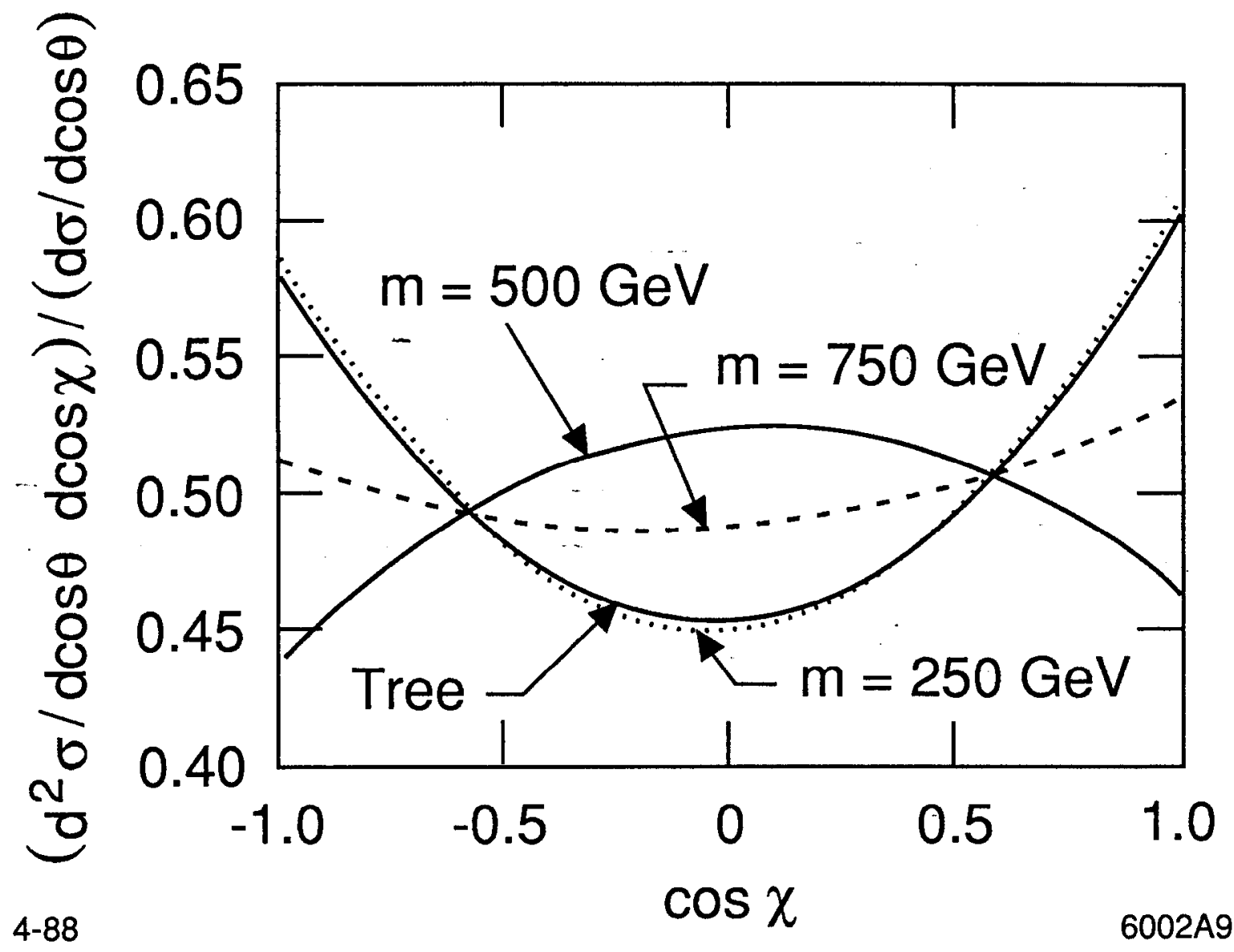

Figure 9. Corrections to the $\chi$ distributions at $\cos \theta=0$, for degenerate fermions.

relatively low energies. The $3 \%$ shift indicated in the figure is just that predicted by (4.6), diluted by the inclusion of the other $W$ polarization states.

Introducing an isospin-breaking mass splitting for the fermion or doublets breaks the standard model's custodial $S U(2)$ symmetry. This is known to lead to a large renormalization of the $\rho$ parameter. In $W$ pair-production, however, such a mass splitting does not generate additional large contributions; rather, its main effect is simply to split the existing peak of the correction term into two. Fig. 11 illustrates this behavior in the differential cross section at $\cos \theta=0$, for 


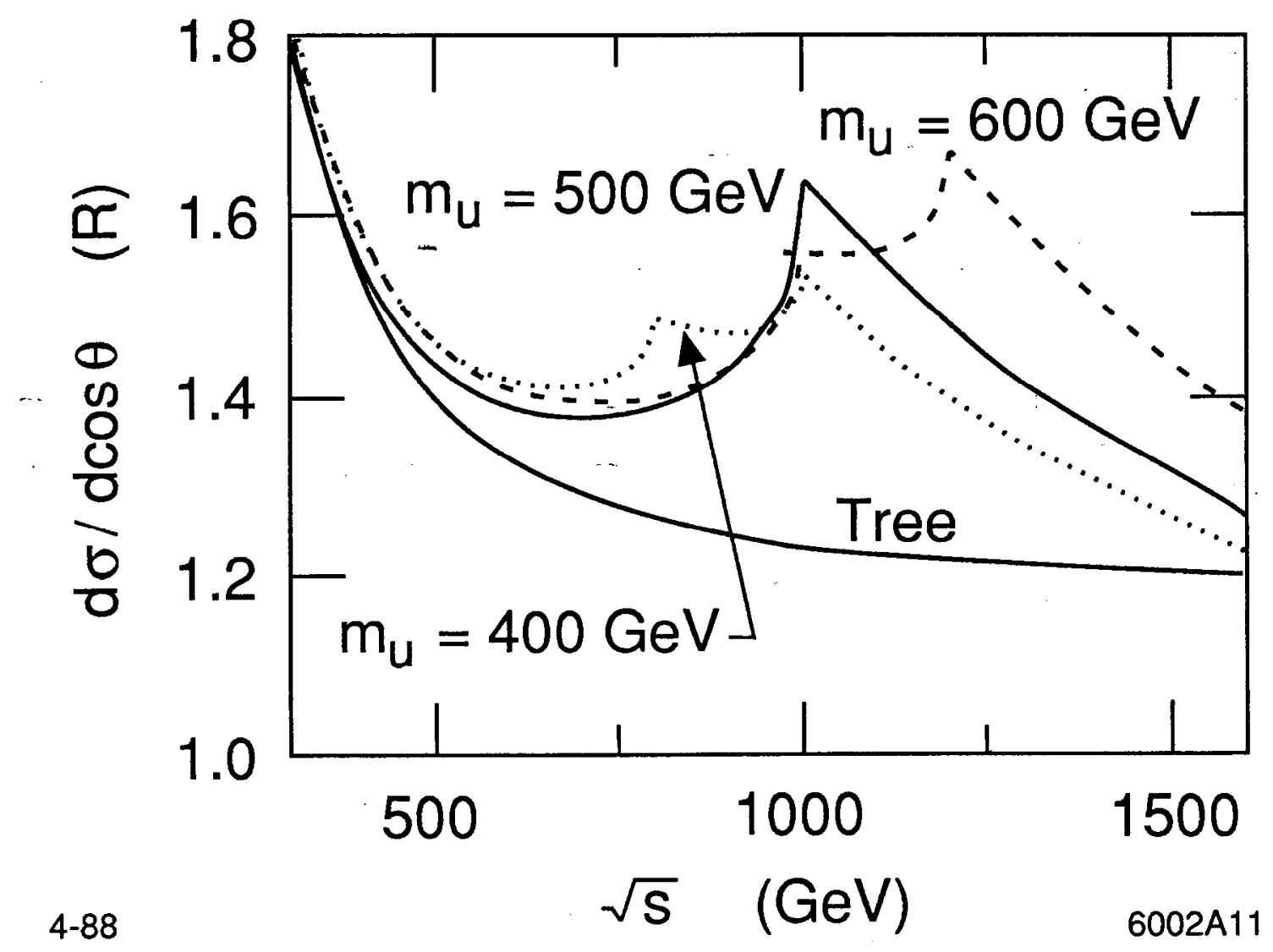

Figure 11. Peak structure for split fermion doublets; $m_{d}=500 \mathrm{GeV}, \cos \theta=0$.

Since the corrections to the tree level cross sections we have found are so large, we must address the question of their reliability. On the one hand, we have seen that the tree-level amplitudes for $W$ pair production are unusually small, due to a cancellation of amplitudes. The large size of the corrections is the result of the fact that they do not exhibit the cancellation. On this ground, we would not expect radiative corrections of still higher order to show a further enhancement.

This argument cannot be complete, however, because the size of our correction term, at threshold and above, increases rapidly with the mass of the heavy gener- 


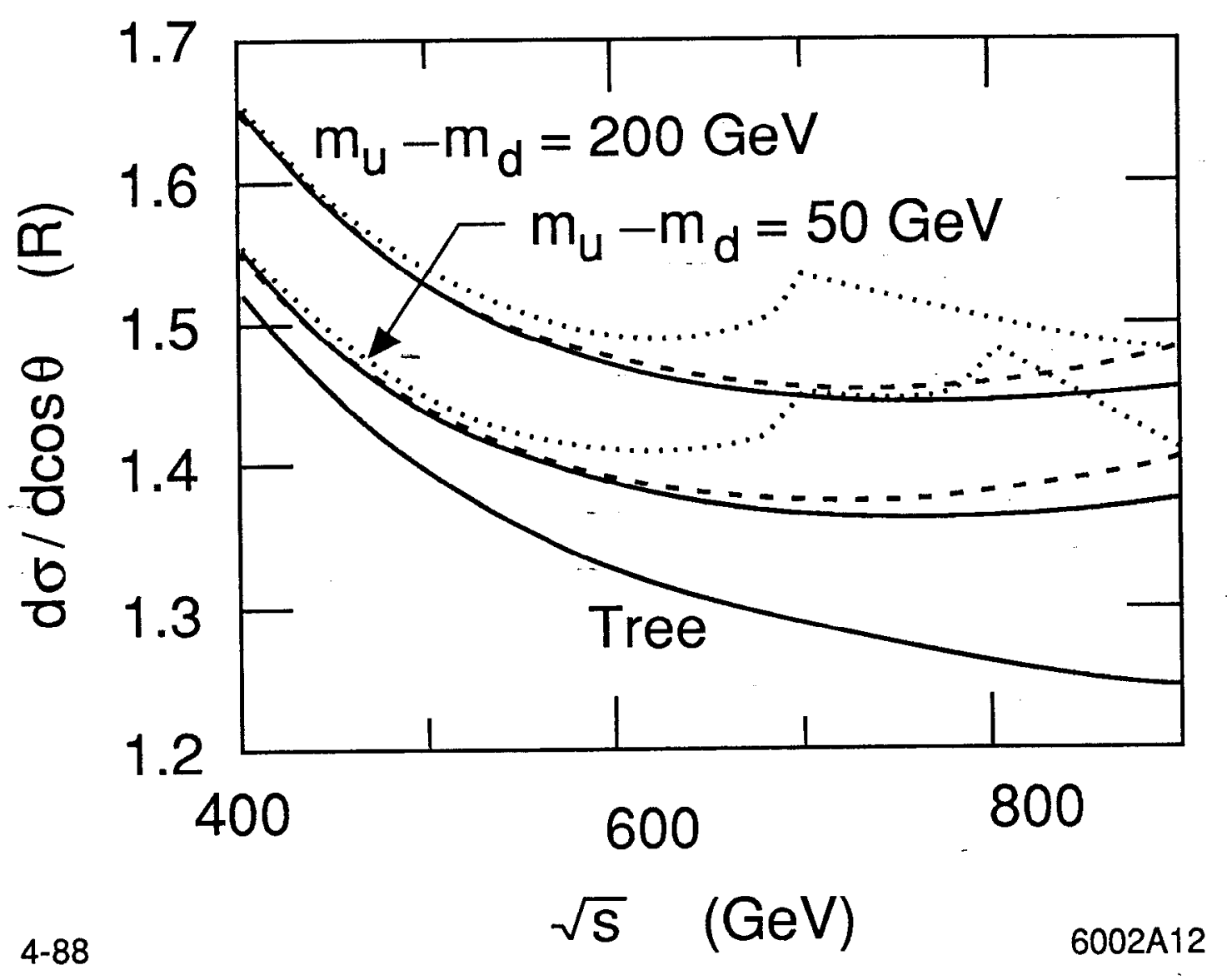

Figure 12. Effects of fermions with an isospin-breaking mass splitting; $\cos \theta=0$. Dotted lines: $m_{d}=350 \mathrm{GeV}$. Dashes: $m_{d}=550 \mathrm{GeV}$. Solid lines: $m_{d}=750 \mathrm{GeV}$.

ation. For example, the residual term (4.8) at very high energies is proportional to $m^{2}$. We can understand this dependence by recalling that the production of longitudinal $W$ bosons at high energy is governed by the Equivalence Theorem, ${ }^{[1,3,52]}$ which states that the production amplitude is equal to that for production of the Goldstone scalars eaten by the $W$ bosons in their mass generation. Indeed, the amplitude for production of scalars through a heavy fermion loop precisely reproduces (4.8), with the prefactor arising from the large fermion-Higgs Yukawa coupling 


$$
\lambda^{2} / 4 \pi=\frac{1}{4 \pi}\left(\frac{m_{f}}{\langle\phi\rangle}\right)^{2}=\frac{\alpha}{2 s_{\theta}^{2}} \cdot \frac{m_{f}^{2}}{m_{W}^{2}} .
$$

It has been shown by Chanowitz, Furman, and Hinchliffe ${ }^{[2]}$ that quarks with masses above $550 \mathrm{GeV}$ cannot be treated perturbatively, since their Yukawa couplings are sufficiently large to violate tree-level unitarity in four-fermion processes. For such heavy quarks, we must expect large corrections to our calculation, proportional to additional powers of the Yukawa coupling, due to virtual Higgs bosons coupling to the fermion loop. Thus, while our calculations should be trustworthy for small enough quark masses (plausibly, for masses as high as $400 \mathrm{GeV}$ ), for higher masses -they should be taken only as an indication of the size of the correction to be expected. We should recall, though, that for the main case of interest, $s<<m^{2}$, we predict an effect which is independent of mass and so extrapolates smoothly into the high-mass regime.

Heavy scalars exhibit much smaller effects than heavy fermions. Scalars with no mass splitting can acquire large mass without coupling to the Higgs sector; at low energies these scalars decouple and at high energies they have no strong couplings to longitudinal $W$ 's. The only significant corrections for scalars, then, are proportional to the mass-squared splittings within isodoublets. Fig. 13 exhibits this behavior; we see that even for $200 \mathrm{GeV}$ mass splittings in either direction, the vertex effect is small and only the $\rho$ parameter effect is observable. Without a mass splitting, it is impossible to separate the corrected and tree-level curves.

Let us finally discuss the size of the corrections we have found in terms of the expected event samples for future $e^{+} e^{-}$colliders. A design for such a collider which is well matched to the requirements of the physics should provide data samples containing a few thousand events for typical annihilation processes; at $\sqrt{s}=1$ $\mathrm{TeV}$, such a sample would correspond to a luminosity of $3 \times 10^{33} \mathrm{~cm}^{-2} \mathrm{sec}^{-1}$ over a running time of a year $\left(3 \times 10^{7} \mathrm{sec}\right)$, for a total integrated luminosity of $9 \times 10^{4}$ $\mathrm{pb}^{-1}$ or $9000 \mathrm{R}^{-1}$. The heavy fermion corrections could be sought either in the gross form of the distribution in $\cos \theta$ or in the shape of the $\cos \chi$ distribution. 


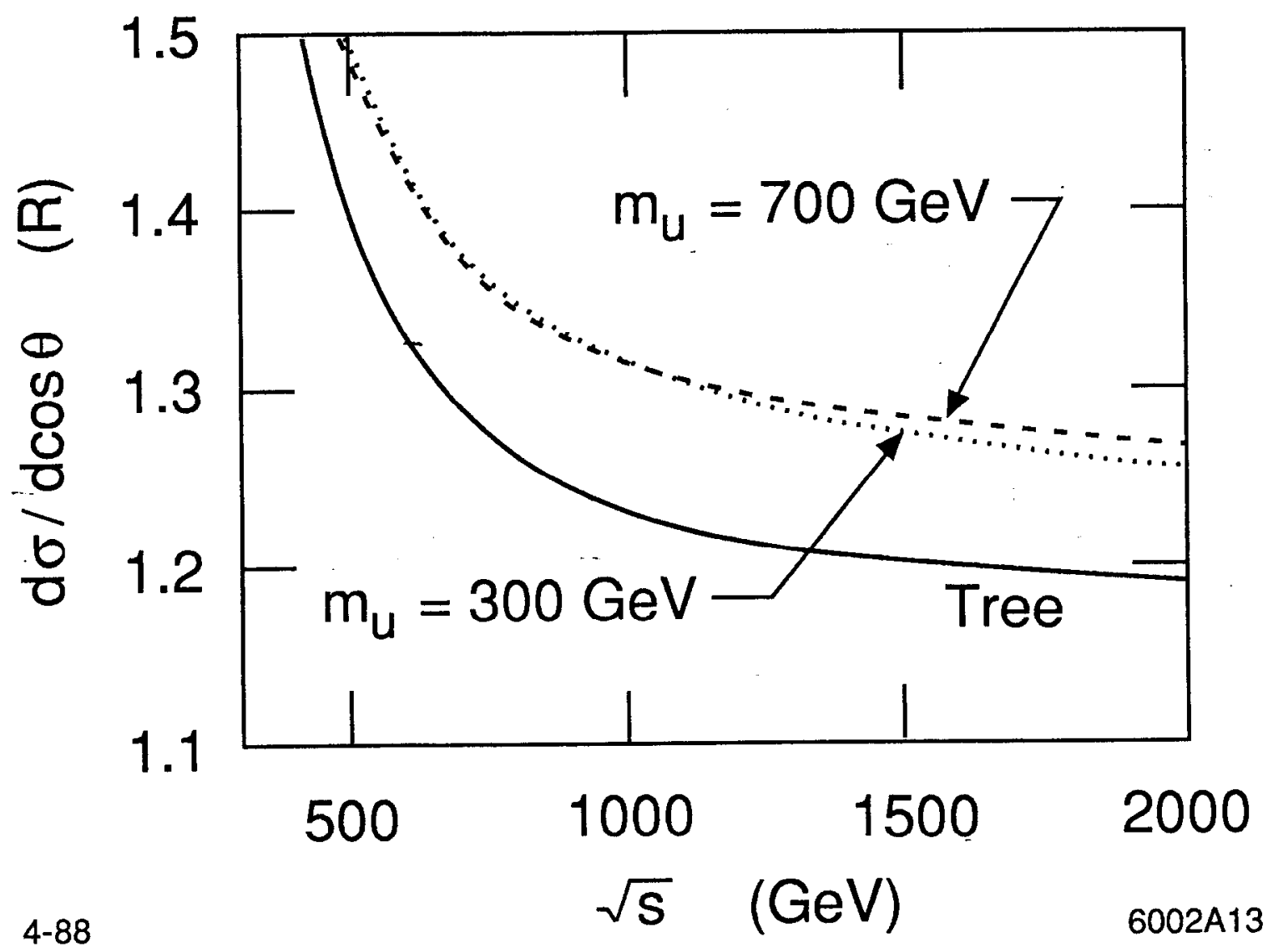

Figure 13. Effect of a supersymmetric generation of scalar partners; $\cos \theta=0, m_{d}=500$ $\mathrm{GeV}$. The upward shift arises almost entirely from the shift in the $\rho$ parameter.

The measurement of $\cos \chi$ requires a leptonic decay. Determining the sign of $\cos \theta$ also requires a lepton or a tightly constrained count of charged particles. However, measures of the differential cross section which are symmetric about $\cos \theta=0$ can be evaluated with essentially the whole sample of $W$ pair events. Our corrections predict a substantial percentage increase in the cross section except at forward angles, suggesting use of the ratio 


$$
R_{\theta}=\int_{|\cos \theta|<0.4} d \cos \theta \frac{d \sigma}{d \cos \theta} / \int_{|\cos \theta|<0.8} d \cos \theta \frac{d \sigma}{d \cos \theta}
$$

This cancels luminosity measurement errors. At $\sqrt{s}=1 \mathrm{TeV}$ with a degenerate generation of fermions of mass $750 \mathrm{GeV}$, using our calculation as an estimate of the effect, we find

$$
R_{\theta}= \begin{cases}0.305 & \text { heavy fermions } \\ 0.289 & \text { standard model }\end{cases}
$$

For the conditions described at the beginning of this paragraph, the numerator of $R_{\theta}$ corresponds to 11,200 events; these should be accepted with efficiency well above $50 \%$. Thus the statistical error on $R_{\theta}$ should be about $1.1 \%$, and the effect indicated in (5.3) should be readily observable at nearly 5 standard deviations. An orthogonal measure of the heavy fermion corrections is

$$
R_{\chi}=\int_{|\cos \chi|<0.6} d \cos \theta d \cos \chi \frac{d \sigma}{d \cos \theta d \cos \chi} / \int d \cos \theta d \cos \chi \frac{d \sigma}{d \cos \theta d \cos \chi}
$$

where the denominator includes all events with semileptonic decays and both integrals are taken over $|\cos \theta|<0.6$. For a heavy generation of fermions of mass 750 $\mathrm{GeV}$ and $\sqrt{s}=1 \mathrm{TeV}$, we predict

$$
R_{\chi}= \begin{cases}0.563 & \text { heavy fermions } \\ 0.543 & \text { standard model }\end{cases}
$$

Roughly $40 \%$ of $W$ pair events will involve one leptonic decay to $\epsilon$ or $\mu$, and these events will be readily reconstructed. Thus, for the same conditions, we expect a statistical error on $R_{\chi}$ of $1.4 \%$. At better than 2.5 standard deviations, this can serve to at least independently confirm an effect discovered in the $\cos \theta$ distribution. New fermions of lower mass, but still above threshold, will produce even larger deviations from the standard model predictions, while higher luminosity would lower the statistical errors. 


\section{Conclusion}

Adding a finite, gauge invariant heavy sector to the standard model gives rise to large effects in $e^{+} e^{-} \rightarrow W^{+} W^{-}$, which we have analyzed in terms of nondecoupling and unitarity delay. Broken global symmetries and large dimensionless parameters are responsible for non-decoupling, while the standard model's gauge cancellations are responsible for unitarity delay. Unitarity delay is most important in the case of longitudinal $W$ 's with their kinematically enhanced $s$ dependence. Since boson vertex corrections generate the main part of the effect, we are able to glean from this process important information which no fermion production -experiment can provide; the three-boson-vertex corrections $\Sigma_{Q+-}$ and $\Sigma_{3+-}$ give new and independent contributions from the virtual states. Effects occurring in four-fermion processes (and most easily measured there), including isospin splitting effects on the $\rho$ parameter and running of coupling constants and boson masses, are all summarized in the running electroweak parameters discussed in Section 3.

At low energy the new contributions are not yet in the asymptotic regime; they disturb the delicate tree-level unitarity cancellation and allow us to probe the nonAbelian structure of the standard model's radiative corrections. At higher energies the cancellations are re-established. For sufficiently heavy fermions or sufficiently split scalars there is also a strong coupling regime; either strong coupling effects or our calculated results will be measurable, with a cross section shift on the order of 0.02 picobarn. 


\section{APPENDIX A: Computation of Differential Cross Sections}

In this appendix, we give some details of the derivation of the general formulae for the $e^{+} e^{-} \rightarrow W^{+} W^{-}$differential cross sections (2.9), (2.13). These formulae follow straightforwardly from (2.8) by inserting explicit forms for the fermion spinors and the $W$ bosons' polarization vectors.

To define the electron spinor matrix elements, choose the electron beam direction as the $\hat{3}$ axis. Then the matrix elements for spinors of definite helicity are given by the simple expression:

$$
\bar{v} \gamma_{\mu} u_{R, L}=\sqrt{s} \cdot\left(\vec{\varepsilon}_{ \pm}, 0\right), \quad \text { where } \quad \vec{\varepsilon}_{ \pm}=\hat{1} \pm i \hat{2}
$$

The upper sign refers to the helicity state $e_{R}^{-}+e_{L}^{+}$, the lower sign to $e_{L}^{-}+e_{R}^{+}$. The $W$ polarization vectors may be specified more directly as

$$
\begin{array}{cc}
\mathcal{E}_{T}^{\alpha}=\left(\vec{\epsilon}_{T}, 0\right) \quad \text { with } \quad \vec{q} \cdot \vec{\epsilon}_{T}=0 & \text { for transverse polarization } \\
\mathcal{E}_{L}^{\alpha}=\frac{1}{m_{W}}\left(q_{0} \frac{\vec{q}}{|\vec{q}|}, i|\vec{q}|\right) & \text { for longitudinal polarization. }
\end{array}
$$

With these choices, it is straightforward though a bit tedious to work out the explicit values of (2.8) and the $t$-channel exchange diagram for each polarization state. This calculation yields the following expression for the $e^{+} e^{-} \rightarrow W^{+} W^{-}$ scattering amplitudes between states of definite helicity:

$$
\mathcal{M}=-i e^{2} \mathcal{A}
$$

where for the various cases of $W$ polarizations:

$$
\begin{aligned}
& \mathcal{A}_{T T}=A_{1} \vec{\varepsilon}_{ \pm} \cdot \hat{q} \vec{\epsilon}_{T}^{-} \cdot \vec{\epsilon}_{T}^{+}+A_{2}\left(\hat{3} \cdot \vec{\epsilon}_{T}^{4} \vec{\varepsilon}_{ \pm} \cdot \vec{\epsilon}_{T}+\hat{3} \cdot \vec{\epsilon}_{T} \vec{\varepsilon}_{ \pm} \cdot \vec{\epsilon}_{T}^{4}\right) \\
& \mathcal{A}_{T L}=A_{3} \vec{\varepsilon}_{ \pm} \cdot \vec{\epsilon}_{T}-A_{4} \hat{3} \cdot \vec{\epsilon}_{T}^{-} \vec{\varepsilon}_{ \pm} \cdot \hat{q} \\
& \mathcal{A}_{L T}=-A_{3} \vec{\varepsilon}_{ \pm} \cdot \vec{\epsilon}_{T}^{+}+A_{4} \hat{3} \cdot \vec{\epsilon}_{T}^{+} \vec{\varepsilon}_{ \pm} \cdot \hat{q} \\
& \mathcal{A}_{L L}=A_{5} \vec{\varepsilon}_{ \pm} \cdot \hat{q}
\end{aligned}
$$


where $\hat{q}$ is a unit vector in the direction of the $W^{-}$momentum, $\vec{\epsilon}_{T}^{ \pm}$are the transverse polarization vectors, and the factors $A_{i}$ are just those listed in (2.10), (2.11).

Squaring this expression and summing over the transverse unit vectors $\vec{\epsilon}_{T}, \vec{\epsilon}_{T}^{+}$ produces precisely the formula (2.9). To obtain (2.13), we require only a small extra piece of analysis. The square of the amplitude for the decay of $W^{-}$to $\ell^{-} \ddot{\nu}$, evaluated in the $W^{-}$rest frame, is proportional to

$$
e^{i *} \cdot\left[\delta^{i j}-n^{i} n^{j}-i \epsilon^{i j k} n^{k}\right] \cdot \epsilon^{j}
$$

where $\vec{\epsilon}$ is the polarization vector of the $W^{-}$and $\vec{n}$ is a unit vector in the direction of the lepton's momentum as viewed from this frame. We may specify the direction of $\vec{n}$ in terms of two angles - the angle $\chi$ and an azimuthal angle $\psi$ about the $\hat{q}$ axis. We may define $\chi$ to be the polar angle between $\vec{n}$ and $\hat{q}$. Although we can obtain interference terms between different polarizations from this formula, wc find it simplest to average over $\chi$; then we may replace in (A.5)

$$
n^{k} \rightarrow \cos \chi \cdot \hat{q}^{k}, \quad n^{i} n^{j} \rightarrow \cos ^{2} \chi \cdot \hat{q}^{i} \hat{q}^{j}+\sin ^{2} \chi \cdot \frac{1}{2}\left(\delta^{i j}-\hat{q}^{i} \hat{q}^{j}\right) .
$$

This simplified form of (A.5) may be combined with the squares of the amplitudes (A.4) and summed over $W^{-}$polarizations, to yield eq. (2.13) in the narrow-width, on-shell approximation. 


\section{APPENDIX B: Explicit Formulae for the $W^{+} W^{-}$Form Factors}

In this appendix, we present explicit expressions for the Feynman diagrams of Fig. 6, and we convert these expressions to formulae for the one-loop corrected form factors, eq. (3.19). We express these formulae in terms of the one-loop integrals defined by Passarino and Veltman, ${ }^{[48]}$ and in terms of a set of reduced Passarino-Veltman functions defined in Appendix C.

\section{B.1. Heavy Fermions}

We consider first the case of one generation of heavy fermions. To cancel “anomalies, we must consider a full generation; our formulae will be written as sums over $f=u_{i}, d_{i}, \nu, \ell$ or doublets $d=\left(u_{i}, d_{i}\right)(\nu, \ell)$, where $i$ runs over 3 colors. When we sum over doublets, the subscripts $u$ and $d$ will denote the up and down components. $Q$ will denote the electric charge of a particle and $I_{3}, Y$ its isospin and hypercharge: $Q=I_{3}+Y$.

The vacuum polarization insertions defined in (3.1) are given in terms of the functions $b_{i}$ defined in appendix $C$ by the following expressions: ${ }^{[32,53]}$

$$
\begin{aligned}
16 \pi^{2} \Pi_{Q Q}^{p}\left(P^{2}\right)= & 8 \sum_{f} Q_{f}^{2}\left[-\frac{\Delta}{6}+\mathbf{b}_{3}\left(P^{2}, m_{f}^{2}, m_{f}^{2}\right)\right] \\
16 \pi^{2} \Pi_{3 Q}^{p}\left(P^{2}\right)= & 4 \sum_{f}\left(Q I_{3}\right)_{f}\left[-\frac{\Delta}{6}+\mathbf{b}_{3}\left(P^{2}, m_{f}^{2}, m_{f}^{2}\right)\right] \\
16 \pi^{2} \Pi_{33}\left(P^{2}\right)=2 \sum_{f}\left(I_{3}\right)_{f}^{2}\left[2 P^{2}\left(-\frac{\Delta}{6}+\mathbf{b}_{3}\left(P^{2}, m_{f}^{2}, m_{f}^{2}\right)\right)\right. & \left.-m_{f}^{2}\left(\Delta+\mathbf{b}_{0}\left(P^{2}, m_{f}^{2}, m_{f}^{2}\right)\right)\right] \\
16 \pi^{2} \Pi_{11}\left(P^{2}\right)= & \sum_{d}\left[2 P^{2}\left(-\frac{\Delta}{6}+\mathbf{b}_{3}\left(P^{2}, m_{u}^{2}, m_{d}^{2}\right)\right)-\frac{m_{u}^{2}+m_{d}^{2}}{2} \Delta\right. \\
& \left.+m_{d}^{2} \mathbf{b}_{1}\left(P^{2}, m_{d}^{2}, m_{u}^{2}\right)+m_{u}^{2} \mathbf{b}_{1}\left(P^{2}, m_{u}^{2}, m_{d}^{2}\right)\right]
\end{aligned}
$$


Here, $\Delta$ is the divergence of dimensional regularization, $\Delta=\pi^{-\left(2-\frac{d}{2}\right)} \cdot \Gamma\left(2-\frac{d}{2}\right) \sim$ $1 /\left(2-\frac{d}{2}\right)-\gamma-\ln \pi$. An arbitrary mass parameter $\ln m_{R}^{2}$, arising from coupling constant dimensions and serving to eliminate dimensionful logarithms, follows $\Delta$ and cancels out along with it. From these formulae, we can immediately assemble expressions for the heavy particle contributions to the running coupling constants, the running $Z$ mass, and the wave function factor $\xi$. For the running couplings,

$$
\begin{aligned}
\frac{1}{e_{*}^{2}\left(P^{2}\right)}-\frac{1}{e_{*}^{2}\left(\mu^{2}\right)} & =-\sum_{f}\left[\frac{8}{16 \pi^{2}} Q_{f}^{2}\left(\mathbf{b}_{3}\left(P^{2}, m_{f}^{2}, m_{f}^{2}\right)-\mathbf{b}_{3}\left(\mu^{2}, m_{f}^{2}, m_{f}^{2}\right)\right)\right] \\
\ldots \frac{1}{g_{*}^{2}\left(P^{2}\right)}-\frac{1}{g_{*}^{2}\left(\mu^{2}\right)} & =-\sum_{f}\left[\frac{4}{16 \pi^{2}}\left(Q I_{3}\right)_{f}\left(\mathbf{b}_{3}\left(P^{2}, m_{f}^{2}, m_{f}^{2}\right)-\mathbf{b}_{3}\left(\mu^{2}, m_{f}^{2}, m_{f}^{2}\right)\right)\right]
\end{aligned}
$$

The factor $\xi$ becomes

$$
\begin{gathered}
(\xi-1)=\frac{g_{*}^{2}}{16 \pi^{2}} \sum_{d}\left[\frac { \partial } { \partial P ^ { 2 } } \left\{2 P^{2} \mathbf{b}_{3}\left(P^{2}, m_{u}^{2}, m_{d}^{2}\right)+m_{u}^{2} \mathbf{b}_{1}\left(P^{2}, m_{u}^{2}, m_{d}^{2}\right)\right.\right. \\
\left.+m_{d}^{2} \mathbf{b}_{1}\left(P^{2}, m_{d}^{2}, m_{u}^{2}\right)\right\}\left.\right|_{P^{2}=-m_{W}^{2}} \\
\left.-4\left\{\frac{1}{2}\left(\frac{1}{2}+Y\right) \mathbf{b}_{3}\left(P^{2}, m_{u}^{2}, m_{u}^{2}\right)+\frac{1}{2}\left(\frac{1}{2}-Y\right) \mathbf{b}_{3}\left(P^{2}, m_{d}^{2}, m_{d}^{2}\right)\right\}\right]
\end{gathered}
$$

We require the running $Z$ mass in the particular form which appears in eq. (3.17); for a generation of fermion doublets this is

$$
\begin{aligned}
M_{Z *}^{2}-m_{Z}^{2} & =\frac{g_{*}^{2}}{16 \pi^{2} c_{*}^{2}} \cdot \sum_{f}\left[8 Q_{f}^{2} m_{Z}^{2} \cdot\left[\mathbf{b}_{3}\left(P^{2}\right)-\mathbf{b}_{3}\left(-m_{Z}^{2}\right)\right]\right. \\
& +4\left(I_{3} Q\right)_{f}\left\{\left[P^{2}+m_{Z}^{2}\left(1-2 s_{*}^{2}\right)\right] \cdot \mathbf{b}_{3}\left(P^{2}\right)+2 m_{Z}^{2} s_{*}^{2} \cdot \mathbf{b}_{3}\left(-m_{Z}^{2}\right)\right\} \\
& \left.-P^{2} \cdot \mathbf{b}_{3}\left(P^{2}\right)-m_{Z}^{2} \cdot \mathbf{b}_{3}\left(-m_{Z}^{2}\right)+\frac{1}{2} m_{f}^{2} \cdot\left[\mathbf{b}_{0}\left(P^{2}\right)-\mathbf{b}_{0}\left(-m_{Z}^{2}\right)\right]\right]
\end{aligned}
$$


The computation of the vertex diagrams, Fig. 6(b), is less straightforward. After performing the Dirac algebra, one must gather terms together into the Lorentz structures given in eq. (2.1), ignoring terms proportional to the electron mass and using the trick in Appendix A of ref. 44 to eliminate additional structures. After this rearrangement, the coefficients of the structures $T_{4}, T_{6}$, and $T_{7}$ disappear as required. Evaluating the integrals using dimensional regularization, we find additional finite terms of the form

$$
\Delta \cdot\left(2-\frac{d}{2}\right) \rightarrow 1
$$

arising from fermion traces. It is essential to keep these terms in order to obtain the unitarity cancellation in the one loop corrections. The final result can be written as follows:

$$
\begin{gathered}
\Sigma_{3+-}=\frac{1}{2} \sum_{\text {doublets }}\left[\left(I_{3}\right)_{d} \cdot H\left(P^{2}, m_{u}^{2}, m_{d}^{2}\right)-\left(I_{3}\right)_{u} \cdot \tilde{H}\left(P^{2}, m_{d}^{2}, m_{u}^{2}\right)\right] \\
\Sigma_{Q+-}=\frac{1}{2} \sum_{\text {doublets }}\left[Q_{d} \cdot\left[H\left(P^{2}, m_{u}^{2}, m_{d}^{2}\right)-G\left(P^{2}, m_{u}^{2}, m_{d}^{2}\right)\right]-\right. \\
\left.Q_{u} \cdot\left[\tilde{H}\left(P^{2}, m_{d}^{2}, m_{u}^{2}\right)-\tilde{G}\left(P^{2}, m_{d}^{2}, m_{u}^{2}\right)\right]\right]
\end{gathered}
$$

where $H=\sum_{i} H^{(i)} \cdot T_{i}$ and $\tilde{H}=H-2 H^{(4)} \cdot T_{4}-2 H^{(5)} \cdot T_{5}$, i.e. $T_{4}$ and $T_{5}$ reverse sign, and similarly with $G$. Finally, in terms of Passarino-Veltman integrals 


$$
\begin{aligned}
16 \pi^{2} G\left(P^{2}, m_{1}^{2}, m_{2}^{2}\right)= & \left(T_{0}-T_{3}-T_{5}\right) \cdot \frac{m_{1}^{2}}{m_{R}^{2}}\left(\mathbf{c}_{2}-\mathbf{c}_{3}\right) \\
16 \pi^{2} H\left(P^{2}, m_{1}^{2}, m_{2}^{2}\right)= & \\
& T_{0}\left(\frac{2}{3}(1-\Delta)+\mathbf{c}_{0}+\mathbf{c}_{1}-\frac{m_{W}^{2}}{m_{R}^{2}}\left(\mathbf{c}_{4}-\mathbf{c}_{5}\right)-\frac{s}{m_{R}^{2}}\left(\mathbf{c}_{6}+\mathbf{c}_{7}\right)\right) \\
- & T_{2} \cdot 4 \cdot \frac{m_{W}^{2}}{m_{R}^{2}} \mathbf{c}_{7} \\
+ & T_{3}\left(\mathbf{c}_{0}-3 \mathbf{c}_{1}-\frac{m_{W}^{2}}{m_{R}^{2}}\left(2 \mathbf{c}_{3}-5 \mathbf{c}_{4}+3 \mathbf{c}_{5}\right)+\frac{s}{m_{R}^{2}}\left(\mathbf{c}_{6}+3 \mathbf{c}_{7}\right)\right) \\
+ & T_{5}\left(-\mathbf{c}_{0}+3 \mathbf{c}_{1}-\frac{m_{W}^{2}}{m_{R}^{2}}\left(\mathbf{c}_{4}-\mathbf{c}_{5}\right)+\frac{s}{m_{R}^{2}}\left(\mathbf{c}_{6}-\mathbf{c}_{7}\right)\right) ;
\end{aligned}
$$

the $\mathbf{c}_{i}$ have arguments $\left(P^{2}, m_{1}^{2}, m_{2}^{2}\right)$.

\section{B.2. HEAVY SCALARS}

We now consider a hypothetical heavy scalar doublet $\Phi=\left(\phi_{u}, \phi_{d}\right)$ with $S U(2) \times$

$U(1)$ quantum numbers $I_{3}= \pm \frac{1}{2}, Q=\left(Q_{u}, Q_{d}\right)$, masses $\left(m_{u}, m_{d}\right)$, and vanishing vacuum expectation value. We obtain vertex corrections

$$
\begin{aligned}
\Sigma_{Q+-} & =\frac{1}{16 \pi^{2}} \times \\
& {\left[Q_{u}\left(\left(\frac{1}{6} \Delta-\mathbf{c}_{1}\right) \cdot T_{0}-2 \frac{m_{W}^{2}}{m_{R}^{2}} \mathbf{c}_{7} \cdot T_{2}+\left(3 \mathbf{c}_{1}-\mathbf{c}_{0}\right) \cdot T_{3}\right)\left(P^{2}, m_{d}^{2}, m_{u}^{2}\right)\right.} \\
& \left.-Q_{d}\left(\left(\frac{1}{6} \Delta-\mathbf{c}_{1}\right) \cdot T_{0}-2 \frac{m_{W}^{2}}{m_{R}^{2}} \mathbf{c}_{7} \cdot T_{2}+\left(3 \mathbf{c}_{1}-\mathbf{c}_{0}\right) \cdot T_{3}\right)\left(P^{2}, m_{u}^{2}, m_{d}^{2}\right)\right] \\
\Sigma_{3+-} & =\frac{1 / 2}{16 \pi^{2}}\left[\left(\left(\frac{1}{6} \Delta-\mathbf{c}_{1}\right) \cdot T_{0}-2 \frac{m_{W}^{2}}{m_{R}^{2}} \mathbf{c}_{7} \cdot T_{2}+\left(3 \mathbf{c}_{1}-\mathbf{c}_{0}\right) \cdot T_{3}\right)\left(P^{2}, m_{u}^{2}, m_{d}^{2}\right)\right. \\
& \left.+\left(\left(\frac{1}{6} \Delta-\mathbf{c}_{1}\right) \cdot T_{0}-2 \frac{m_{W}^{2}}{m_{R}^{2}} \mathbf{c}_{7} \cdot T_{2}+\left(3 \mathbf{c}_{1}-\mathbf{c}_{0}\right) \cdot T_{3}\right)\left(P^{2}, m_{d}^{2}, m_{u}^{2}\right)\right]
\end{aligned}
$$


and two-point corrections ${ }^{[53,54]}$

$$
\begin{aligned}
& \begin{aligned}
& \Pi_{Q Q}^{p}=-\frac{1}{16 \pi^{2}}\left[Q_{u}^{2} \cdot\left(\frac{\Delta}{3}+4 \mathbf{b}_{3}+\mathbf{b}_{0}\right)\left(P^{2}, m_{u}^{2}, m_{u}^{2}\right)\right. \\
&+\left.Q_{d}^{2} \cdot\left(\frac{\Delta}{3}+4 \mathbf{b}_{3}+\mathbf{b}_{0}\right)\left(P^{2}, m_{d}^{2}, m_{d}^{2}\right)\right] \\
& \Pi_{3 Q}^{p}=-\frac{1}{16 \pi^{2}} \cdot \frac{1}{2}\left[Q_{u} \cdot+\frac{\Delta}{3}+4 \mathbf{b}_{3}+\mathbf{b}_{0}\right)\left(P^{2}, m_{u}^{2}, m_{u}^{2}\right)
\end{aligned} \\
& \left.\quad-Q_{d} \cdot\left(\frac{\Delta}{3}+4 \mathbf{b}_{3}+\mathbf{b}_{\theta}\right)\left(P^{2}, m_{d}^{2}, m_{d}^{2}\right)\right] \\
& \Pi_{33}=-\frac{1}{16 \pi^{2}} \frac{P^{2}}{4}\left[\frac{2 \Delta}{3}+\left(4 \mathbf{b}_{3}+\mathbf{b}_{0}\right)\left(P^{2}, m_{u}^{2}, m_{u}^{2}\right)+\left(4 \mathbf{b}_{3}+\mathbf{b}_{0}\right)\left(P^{2}, m_{d}^{2}, m_{d}^{2}\right)\right] \\
& \Pi_{11}=-\frac{1}{16 \pi^{2}} \cdot \frac{1}{2}\left[P^{2}\left(\frac{\Delta}{3}+4 \mathbf{b}_{3}+\mathbf{b}_{0}\right)\left(P^{2}, m_{u}^{2}, m_{d}^{2}\right)\right. \\
& \left.\quad+\left(m_{d}^{2}-m_{u}^{2}\right) \cdot\left[\mathbf{b}_{1}\left(P^{2}, m_{d}^{2}, m_{u}^{2}\right)-\mathbf{b}_{1}\left(P^{2}, m_{u}^{2}, m_{d}^{2}\right)\right]\right] .
\end{aligned}
$$

For the case of a full generation of superpartners, we can sum over sleptons and squarks. 


\section{APPENDIX C: Reduced Passarino-Veltman Functions}

All higher Passarino-Veltman functions may be uniquely decomposed into linear combinations of the scalar integrals $B_{0}$ and $C_{0}$, for which closed form expressions are known. ${ }^{[\mathbf{4 8 , 4 9 ]}}$ The decomposition algorithm has been implemented in an algebraic manipulation program; ${ }^{[51]}$ for purposes of asymptotic analysis, however, we have found it convenient to define reduced Passarino-Veltman functions representing finite, dimensionless parts of two- and three-point one-loop integrals. All of these functions include an arbitrary mass scale $m_{R}$, which cancels out of all physical results. For the two-point functions, it is straightforward to determine the asymptotic forms of these functions. For the three-point functions, the asymptotic analysis requires some effort, and so we have catalogued the required formulae.

The functions $\mathbf{b}_{i}\left(P^{2}, m_{1}^{2}, m_{2}^{2}\right)$ which appear in Appendix $\mathrm{C}$ are defined as follows:

$$
\begin{gathered}
{\left[\mathbf{b}_{0}, \mathbf{b}_{1}, \mathbf{b}_{3}\right]=\int_{0}^{1} d x \log \left(\left[x m_{1}^{2}+(1-x) m_{2}^{2}+x(1-x) P^{2}-i \epsilon\right] / m_{R}^{2}\right)} \\
\cdot[-1, x, x(1-x)] .
\end{gathered}
$$

These functions are related to the corresponding Passarino-Veltman integrals ${ }^{[48]}$ by

$$
\begin{aligned}
& B_{0}\left(m_{2}, m_{1}\right)=\mathbf{b}_{0}\left(m_{1}, m_{2}\right)+\left(\Delta-\ln m_{R}^{2}\right) \\
& B_{1}\left(m_{2}, m_{1}\right)=\mathbf{b}_{1}\left(m_{1}, m_{2}\right)-\frac{1}{2}\left(\Delta-\ln m_{R}^{2}\right) \\
& B_{3}\left(m_{2}, m_{1}\right)=\mathbf{b}_{3}\left(m_{1}, m_{2}\right)-\frac{1}{6}\left(\Delta-\ln m_{R}^{2}\right) .
\end{aligned}
$$

with $B_{3}=B_{21}+B_{1} ; B_{3}$ and $B_{0}$ are symmetric in $m_{1}^{2}, m_{2}^{2}$. 
Passarino and Veltman's $C$ functions are defined by

$$
\begin{aligned}
C_{0, \mu, \mu \nu, \mu \nu \rho}\left(q^{2}, \bar{q}^{2}, P^{2}, m_{1}^{2}, m_{2}^{2}, m_{3}^{2}\right) \equiv \\
\int \frac{d^{d} k}{i \pi^{2}} \frac{\left\{1, k_{\mu}, k_{\mu} k_{\nu}, k_{\mu} k_{\nu} k_{\rho}\right\}}{\left(k^{2}+m_{1}^{2}\right)\left[(k+q)^{2}+m_{2}^{2}\right]\left[(k+P)^{2}+m_{3}^{2}\right]}
\end{aligned}
$$

and can be written in terms of form factors;

$$
\begin{aligned}
C_{\mu}= & \bar{q}_{\mu} C_{11}+q_{\mu} C_{12} \\
C_{\mu \nu} & =\bar{q}_{\mu} \bar{q}_{\nu} C_{21}+q_{\mu} q_{\nu} C_{22}+\{q \bar{q}\}_{\mu \nu} C_{23}+\delta_{\mu \nu} C_{24} \\
C_{\mu \nu \rho} & =\bar{q}_{\mu} \bar{q}_{\nu} \bar{q}_{\rho} C_{31}+q_{\mu} q_{\nu} q_{\rho} C_{32} \\
& +\{q \bar{q} \bar{q}\}_{\mu \nu \rho} C_{33}+\{\bar{q} q q\}_{\mu \nu \rho} C_{34} \\
& +\{\bar{q} \delta\}_{\mu \nu \rho} C_{35}+\{q \delta\}_{\mu \nu \rho} C_{36}
\end{aligned}
$$

with braces summing over distinct permutations. $P=q+\bar{q}$ always.

In the present case we may set $m_{1}^{2}=m_{3}^{2} \rightarrow m_{2}^{2}$ and $m_{2}^{2} \rightarrow m_{1}^{2}$; then we define the reduced Passarino-Veltman functions $\mathbf{c}_{i}\left(P^{2}, m_{1}^{2}, m_{2}^{2}\right)$ in terms of the denominator

$$
D=z m_{1}^{2}+(1-z) m_{2}^{2}-z(1-z) m_{W}^{2}+x y P^{2}-i \epsilon
$$

as follows:

$$
\begin{aligned}
{\left[\mathbf{c}_{0}, \mathbf{c}_{1}\right] } & =\int d x d y d z \delta(x+y+z-1) \log \left(D / m_{R}^{2}\right) \\
& \times[1, z] \\
{\left[\mathbf{c}_{2}, \mathbf{c}_{3}, \mathbf{c}_{4}, \mathbf{c}_{5}, \mathbf{c}_{6}, \mathbf{c}_{7}\right] } & =\int d x d y d z \delta(x+y+z-1)\left(\frac{m_{R}^{2}}{D}\right) \\
& \times\left[1, z, z^{2}, z^{3}, x y, x y z\right] .
\end{aligned}
$$


Note that

$$
\left[\mathbf{c}_{6}, \mathbf{c}_{7}\right]=\frac{m_{R}^{2}}{s}\left[-\frac{1}{2},-\frac{1}{6}\right]+\frac{m_{2}^{2}}{s}\left[\mathbf{c}_{2}, \mathbf{c}_{3}\right]+\frac{\left(m_{1}^{2}-m_{2}^{2}-m_{W}^{2}\right)}{s}\left[\mathbf{c}_{3}, \mathbf{c}_{4}\right]+\frac{m_{W}^{2}}{s}\left[\mathbf{c}_{4}, \mathbf{c}_{5}\right]
$$

These functions are related to the corresponding Passarino-Veltman integrals by

$$
\begin{aligned}
& m_{R}^{2} \cdot C_{0}=\mathbf{c}_{2} \\
& m_{R}^{2} \cdot C_{11,12}=-\frac{1}{2}\left(\mathbf{c}_{2} \pm \mathbf{c}_{3}\right) \\
& m_{R}^{2} \cdot C_{21}=\frac{1}{2}\left(\mathbf{c}_{2}+\mathbf{c}_{4}\right)-\mathbf{c}_{6} \\
& m_{R}^{2} \cdot C_{22}=\frac{1}{2}\left(\mathbf{c}_{2}+\mathbf{c}_{4}\right)-\mathbf{c}_{3}-\mathbf{c}_{6} \\
& m_{R}^{2} \cdot C_{23}=\frac{1}{2}\left(\mathbf{c}_{2}-\mathbf{c}_{3}\right)-\mathbf{c}_{6} \\
& m_{R}^{2} \cdot C_{31}=-\frac{1}{2}\left(\mathbf{c}_{2}+\mathbf{c}_{5}\right)+\frac{3}{2}\left(\mathbf{c}_{6}+\mathbf{c}_{7}\right) \\
& m_{R}^{2} \cdot C_{32}=-\frac{1}{2}\left(\mathbf{c}_{2}-3 \mathbf{c}_{3}+3 \mathbf{c}_{4}-\mathbf{c}_{5}\right)+\frac{3}{2}\left(\mathbf{c}_{6}-\mathbf{c}_{7}\right) \\
& m_{R}^{2} \cdot C_{33}=-\frac{1}{2}\left(\mathbf{c}_{2}-\mathbf{c}_{3}\right)+\frac{3}{2} \mathbf{c}_{6}+\frac{1}{2} \mathbf{c}_{7} \\
& m_{R}^{2} \cdot C_{34}=-\frac{1}{2}\left(\mathbf{c}_{2}+\mathbf{c}_{4}\right)+\mathbf{c}_{3}+\frac{3}{2} \mathbf{c}_{6}-\frac{1}{2} \mathbf{c}_{7} \\
& C_{24}=-\frac{1}{2} \mathbf{c}_{0}+\frac{1}{4}\left(\Delta-\ln m_{R}^{2}\right) \\
& C_{35}=\frac{1}{4}\left(\mathbf{c}_{0}+\mathbf{c}_{1}\right)-\frac{1}{6}\left(\Delta-\ln m_{R}^{2}\right) \\
& C_{36}\left(\mathbf{c}_{0}-\mathbf{c}_{1}\right)-\frac{1}{12}\left(\Delta-\ln m_{R}^{2}\right)
\end{aligned}
$$

where

$$
\begin{aligned}
C_{i j} & =C_{i j}\left(-m_{W}^{2},-m_{W}^{2}, P^{2}, m_{2}^{2}, m_{1}^{2}, m_{2}^{2}\right) \\
\mathbf{c}_{i} & =\mathbf{c}_{i}\left(P^{2}, m_{1}^{2}, m_{2}^{2}\right) .
\end{aligned}
$$

We reduce the integrations over three Feynman parameters to one parameter 
integrations for numerical analysis and asymptotic expression.

$$
\begin{aligned}
& {\left[\mathbf{c}_{0}, \mathbf{c}_{1}\right]=\int_{0}^{1} d z\left\{(1-z) \cdot\left(\ln \frac{A}{m_{R}^{2}}-2-i \pi \theta(-A)\right)+|R(z, s)|^{1 / 2} \cdot K(z)\right\} \cdot[1, z]} \\
& {\left[\mathbf{c}_{2}, \mathbf{c}_{3}, \mathbf{c}_{4}, \mathbf{c}_{5}\right]=\int_{0}^{1} d z \frac{2 m_{R}^{2}}{s} \cdot|R(z, s)|^{-1 / 2} \cdot Q(z) \cdot K(z) \cdot\left[1, z, z^{2}, z^{3}\right]}
\end{aligned}
$$

where we define

$$
\begin{aligned}
& A(z)=z m_{1}^{2}+\left(1^{-}-z\right) m_{2}^{2}-z(1-z) m_{W}^{2} \\
& R(z, s)=\frac{4 A}{s}-(1-z)^{2} \\
& Q(z)=\left\{\begin{array}{ccc}
1 & \text { for } & z>z_{+} \\
-1 & \text { for } & z<z_{+}
\end{array}\right. \\
& K(z)=\left\{\begin{array}{l}
2 \arctan \left[(1-z)|R(z, s)|^{-1 / 2}\right] \quad \text { for } \quad z_{+}<z \leq 1 \\
\ln \left[\frac{(1-z)+|R(z, s)|^{1 / 2}}{(1-z)-|R(z, s)|^{1 / 2}}\right]-i \pi \cdot \theta(A) \quad \text { for } \quad 0 \leq z<z_{+}
\end{array}\right.
\end{aligned}
$$

and $z_{+}$, a solution for $R(z, s)=0$, is given by

$$
\begin{aligned}
z_{+}= & \frac{s+2\left(m_{1}^{2}-m_{2}^{2}-m_{W}^{2}\right)}{s-4 m_{W}^{2}} \\
& \quad-\frac{2}{s-4 m_{W}^{2}} \cdot\left[m_{1}^{2} s+\left(m_{1}^{2}-m_{2}^{2}\right)^{2}-2 m_{W}^{2}\left(m_{1}^{2}+m_{2}^{2}\right)+m_{W}^{4}\right]^{1 / 2} .
\end{aligned}
$$

For present purposes, we may disregard the imaginary parts. 
For large and small values of $s$, the functions $c_{i}$ take the following asymptotic forms. We always assume that the mass difference between $m_{1}$ and $m_{2}$ is small and set $\Delta m^{2}=m_{1}^{2}-m_{2}^{2}, m^{2}=\frac{1}{2}\left(m_{1}^{2}+m_{2}^{2}\right)$, with $\Delta m^{2} \ll m^{2}$. Then for $m_{W}^{2} \ll s \ll m^{2}$

$$
\begin{aligned}
& \mathbf{c}_{0}=\frac{1}{2} \ln \frac{s}{m_{R}^{2}}-\frac{1}{12} \frac{\Delta m^{2}}{m^{2}}-\frac{1}{24} \frac{s}{m^{2}} \\
& \mathbf{c}_{1}=\frac{1}{6} \ln \frac{s}{m_{R}^{2}}-\frac{1}{120} \frac{s}{m^{2}} \\
& \mathbf{c}_{2}=\frac{m_{R}^{2}}{m^{2}}\left[\frac{1}{2}+\frac{1}{12} \frac{\Delta m^{2}}{m^{2}}+\frac{1}{24} \frac{s}{m^{2}}\right] \\
& \mathbf{c}_{3}=\frac{m_{R}^{2}}{m^{2}}\left[\frac{1}{6}+\frac{1}{120} \frac{s}{m^{2}}\right] \\
& \mathbf{c}_{4}=\frac{m_{R}^{2}}{m^{2}}\left[\frac{1}{12}-\frac{1}{120} \frac{\Delta m^{2}}{m^{2}}+\frac{1}{360} \frac{s}{m^{2}}\right] \\
& \mathbf{c}_{5}=\frac{m_{R}^{2}}{m^{2}}\left[\frac{1}{20}-\frac{1}{120} \frac{\Delta m^{2}}{m^{2}}+\frac{1}{840} \frac{s}{m^{2}}\right] \\
& \mathbf{c}_{6}=\frac{m_{R}^{2}}{m^{2}}\left[\frac{1}{24}+\frac{1}{80} \frac{\Delta m^{2}}{m^{2}}+\frac{1}{180} \frac{s}{m^{2}}\right] \\
& \mathbf{c}_{7}=\frac{m_{R}^{2}}{m^{2}}\left[\frac{1}{120}+\frac{1}{720} \frac{\Delta m^{2}}{m^{2}}+\frac{1}{1260} \frac{s}{m^{2}}\right] .
\end{aligned}
$$

When $s>m^{2}$, dropping $\Delta m^{2} / m^{2}$ and non-asymptotic terms, 


$$
\begin{aligned}
& \mathbf{c}_{0}=\frac{1}{2} \ln \frac{s}{m_{R}^{2}}-\frac{3}{2} \\
& -\frac{m^{2}}{s}\left[\frac{1}{2}\left[\ln ^{2} \frac{s}{m^{2}}-\pi^{2}\right]+\ln \frac{s}{m^{2}}+1\right] \\
& c_{1}=\frac{1}{6} \ln \frac{s}{m_{R}^{2}}-\frac{11}{18} \\
& -\frac{m^{2}}{s}\left[\frac{1}{2}\left[\ln ^{2} \frac{s}{m^{2}}-\pi^{2}\right]-\ln \frac{s}{m^{2}}+3\right] \\
& \frac{s}{m_{R}^{2}} \cdot \mathbf{c}_{2}=-\frac{1}{2}\left[\ln ^{2} \frac{s}{m^{2}}-\pi^{2}\right] \\
& +\frac{m^{2}}{s}\left[2 \ln \frac{s}{m^{2}}\right] \\
& \frac{s}{m_{R}^{2}} \cdot \mathrm{c}_{3}=-\frac{1}{2}\left[\ln ^{2} \frac{s}{m^{2}}-\pi^{2}\right]+2 \ln \frac{s}{m^{2}}-4 \\
& +\frac{m^{2}}{s}\left[-2 \ln \frac{s}{m^{2}}-4\right] \\
& \frac{s}{m_{R}^{2}} \cdot \mathbf{c}_{4}=-\frac{1}{2}\left[\ln ^{2} \frac{s}{m^{2}}-\pi^{2}\right]+3 \ln \frac{s}{m^{2}}-7 \\
& +\frac{m^{2}}{s}\left[-\left[\ln ^{2} \frac{s}{m^{2}}-\pi^{2}\right]-4 \ln \frac{s}{m^{2}}-6\right] \\
& \frac{s}{m_{R}^{2}} \cdot c_{5}=-\frac{1}{2}\left[\ln ^{2} \frac{s}{m^{2}}-\pi^{2}\right]+\frac{11}{3} \ln \frac{s}{m^{2}}-\frac{85}{9} \\
& +\frac{m^{2}}{s}\left[-3\left[\ln ^{2} \frac{s}{m^{2}}-\pi^{2}\right]-18\right] \\
& \frac{s}{m_{R}^{2}} \cdot \mathbf{c}_{6}=-\frac{1}{2}+\frac{m^{2}}{s}\left[-\frac{1}{2}\left[\ln ^{2} \frac{s}{m^{2}}-\pi^{2}\right]\right] \\
& \frac{s}{m_{R}^{2}} \cdot c_{7}=-\frac{1}{6}+\frac{m^{2}}{s}\left[-\frac{1}{2}\left[\ln ^{2} \frac{s}{m^{2}}-\pi^{2}\right]+2 \ln \frac{s}{m^{2}}-4\right] \text {. }
\end{aligned}
$$


III. MODULAR INVARIANT PARTITION FUNCTIONS ON NONSIMPLY CONNECTED GROUP MANIFOLDS 


\section{Preliminaries}

\subsection{Conformal Field Theories on the Plane ${ }^{[6]}$}

Conformal field theories are quantum field theories with conformal symmetries. The Poincare symmetry is extended to the conformal symmetry by requiring that the action be scale invariant. Since translational invariance under $x^{\mu} \rightarrow x^{\mu}+a^{\mu}$ gives the energy-momentum tensor $T_{\mu \nu}$ as the corresponding conserved current, conservation of the dilatation current $D_{\mu}=T_{\mu \nu} x^{\nu}$ implies that the energy momentum tensor is traceless. Using these two conditions on the energy-momentum tensor, we can construct additional currents of the form $J_{\mu}^{f}=T_{\mu \nu} f^{\nu}(x)$. If the functional parameter $f^{\nu}(x)$ satisfies

$$
\partial_{\mu} f_{\nu}+\partial_{\nu} f_{\mu}=\frac{2}{d} g_{\mu \nu}(\partial \cdot f)
$$

the currents $J_{\mu}^{f}$ are conserved and generate the generalized conformal group. Under the generalized coordinate transformations $x^{\mu} \rightarrow x^{\prime \mu}=x^{\mu}+f^{\mu}(x)$, the metric $g_{\mu \nu}$ transforms according to (1.1),

$$
g_{\mu \nu}(x) \rightarrow g_{\mu \nu}^{\prime}\left(x^{\prime}\right)=\Omega(x) g_{\mu \nu}(x)
$$

where $d$ denotes the dimension of space-time and $\Omega(x)=1+(2 / d)(\partial \cdot f)$. Eq. $(1.2)$ shows that the transformation preserves angles between two vectors defined on the space-time, which is the original definition of a conformal transformation.

In $d>2$, the only non-trivial solutions of (1.1) are $f_{\mu}(x)=a_{\mu}, x_{\mu}, \omega_{\mu}{ }^{\nu} x_{\nu}$ and $b_{\mu} x^{2}-2 x_{\mu} b \cdot x$ where $a$ and $b$ are constant vectors. These form the finite conformal group: the Poincare group, plus scale transformations and special conformal transformations. In $d=2$ with the Eucleadian metric $g_{\mu \nu}=\delta_{\mu \nu}$, any holomorphic and anti-holomorphic functions, $f(z)$ and $\bar{f}(\bar{z})$ satisfy (1.1), where $z, \bar{z}=x_{1} \pm i x_{2}$. Therefore, the conformal group in two dimensions is infinite dimensional and is 
generated by two independent coordinate transformations,

$$
z \rightarrow w(z), \quad \bar{z} \rightarrow \bar{w}(\bar{z})
$$

In two dimensions, scale invariance implies conformal invariance. The energymomentum tensor has two non-vanishing components $T_{z \bar{z}}=T(z)$ and $T_{\bar{z} \bar{z}}=\bar{T}(\bar{z})$, which become generators of the two conformal transformations. Using (1.3), we define $z=\exp \left(x_{2}-i x_{1}\right), \quad \bar{z}=\exp \left(-x_{2}-i x_{1}\right)$ so that as time component $x_{2}$ goes to $-\infty, z$ goes to 0 . This is called radial quantization because equal radius means equal time in this complex coordinate. Since these holomorphic and anti-holomorphic sectors are completely independent of each other, we can concentrate on holomorphic sector only from now on. However, these two sectors must eventually be combined together to fulfill the full consistency requirements on the theory.

The conformal field theories are classified by an anomaly term called the central charge of the conformal algebra, which arises from operator product expansion of $T(z)$. The general form of the operator product is,

$$
T(z) T(w) \sim \frac{c / 2}{(z-w)^{4}}+\frac{2}{(z-w)^{2}} T(w)+\frac{1}{z-w} \partial T(w),
$$

where $c$ denotes this central charge. Using a Laurent expansion fo the energymomentum tensor, one can define the mode $L_{n}$ as $L_{n}=\oint \frac{d z}{2 \pi i} z^{n+1} T(z)$. From (1.4), we get the Virasoro algebra,

$$
\left[L_{n}, L_{m}\right]=(n-m) L_{n+m}+\frac{c}{12}\left(n^{3}-n\right) \delta_{n+m, 0}
$$

We can define two vacuum states $|0\rangle,\langle 0|$ at $z=0, \infty$. In order to make sense out of $T(z)|0\rangle$ as $z \rightarrow 0,\langle 0| T^{\dagger}(z)$ as $z \rightarrow \infty$, we must require

$$
L_{n}|0\rangle=0, \quad\langle 0| L_{n}^{\dagger}=\langle 0| L_{-n}=0, \quad n \geq-1
$$

The operators $L_{1}, L_{0}, L_{-1}$ generate a $S L(2, C)$ subalgebra of the Virasoro algebra which preserves the vacuum. The primary field $\phi_{h}(z)$ generates highest weight 
state $|h\rangle \equiv \phi_{h}(0)|0\rangle$ which satisfies

$$
L_{0}|h\rangle=h|h\rangle \quad L_{n}|h\rangle=0, \quad n>0 .
$$

Here, $h$ denotes the conformal dimension of the primary field. From the highest weight state $|h\rangle$, decendents are generated as a form of $L_{-n_{1}} L_{-n_{2}} \cdots L_{-n_{k}}|h\rangle$ ( all $\left.n_{i}>0\right)$. The sum $N=\sum_{i=1}^{k} n_{i}$ is called the level of the decendent field. The complete set of states descended from $|h\rangle$ is called a Verma module. A Verma module is a (possibly reducible) representation of the Virasoro algebra that is characterized by the central charge $c$ and the dimension $h$ of the highest-weight state.

The correlation functions of quantum fields are central objects to compute in quantum field theories. The standard formulation for this computation is either canonical quantization formalism or path-integral method based on the given action. However, if the theory is interacting, these formalism can provide solutions only perturbatively. Meanwhile, the two-dimensional conformal field theories promise a new possibility for exact solutions for the quantum field theories. Correlation functions for the primary fields $\phi_{\boldsymbol{i}}(z, \bar{z})$ should satisfy conformal Ward identities from the invariance of vacuum under $S L(2, C)$.

$$
\begin{array}{r}
\sum_{j=1}^{n} \partial_{z_{j}}\left\langle\phi_{i_{1}}\left(z_{1}, \bar{z}_{1}\right) \cdots \phi_{i_{n}}\left(z_{n}, \bar{z}_{n}\right)\right\rangle=0 \\
\sum_{j=1}^{n}\left[z_{j} \partial_{z_{j}}+h_{i_{j}}\right]\left\langle\phi_{i_{1}}\left(z_{1}, \bar{z}_{1}\right) \cdots \phi_{i_{n}}\left(z_{n}, \bar{z}_{n}\right)\right\rangle=0 \\
\sum_{j=1}^{n}\left[z_{j}^{2} \partial_{z_{j}}+2 z_{j} h_{i_{j}}\right]\left\langle\phi_{i_{1}}\left(z_{1}, \bar{z}_{1}\right) \cdots \phi_{i_{n}}\left(z_{n}, \bar{z}_{n}\right)\right\rangle=0
\end{array}
$$

Correlators for the decendents can be derived from those of primaries using the conformal Ward identities. Equations (1.8) completely characterize one, two, and 
three-point correlation functions up to some constants. These constants are conformal dimensions of primary fields $h_{i}$ and generalized three point couplings $C_{i j k}$. From these solutions, we can deduce the operator product expansions

$$
\phi_{i}(z, \bar{z}) \cdot \phi_{j}(w, \bar{w})=\frac{C_{i j k}}{(z-w)^{h_{i}+h_{j}-h_{k}}(\bar{z}-\bar{w})^{h_{i}+\bar{h}_{j}-\bar{h}_{k}}}\left[\phi_{k}(w, \bar{w})+\cdots\right]
$$

where the dots denote a sum over an infinite set of decendents with corresponding coefficients which, in principle, can be completely determined by the given input parameters, $h_{i}$ and $C_{i j k}$. Using these operator product expansions, we can compute any correlation function. For example, the four-point function can be written down as

$$
\left\langle\phi_{i}\left(z_{1}, \bar{z}_{1}\right) \cdots \phi_{l}\left(z_{4}, \bar{z}_{4}\right)\right\rangle=\sum_{p} \mathcal{F}_{k l}^{j i}(p \mid \eta) \overline{\mathcal{F}}_{l k}^{i j}(p \mid \bar{\eta})
$$

where $\mathcal{F}_{k l}^{j i}\left(\overline{\mathcal{F}}_{l k}^{i j}\right)$ are called as (anti-)holomorphic conformal blocks. In computing correlation functions, there is an arbitrariness in the order in taking the operator product expansion. Since the final result should not depend on the arbitrariness, we get the associativity condition for the operator expansion product algebra which goes under the name of duality, or crossing symmetry.

The problem of solving the conformal field theories completely reduces to that of computing conformal blocks $\mathcal{F}_{i j}^{k l}$ for all the decendents for the given primaries. This is not easy because conformal blocks involve a sum over an infinite number of decendent fields. In special cases, however, we can derive differential equations for the conformal blocks. The differential equations arise from degenerate representations of the conformal algebra. A degenerate representation of the Virasoro algebra is a representation that contains a null state, a state which is both primary and decendent state at the same time. Then $|\chi\rangle$ is a null state if

$$
L_{0}|\chi\rangle=(h+N)|\chi\rangle, \quad L_{n}|\chi\rangle=0 \quad \text { for } n>0 .
$$

These conditions imply that $|\chi\rangle$ has zero norm. A typical example of null state is 
given by

$$
|\chi\rangle=\left[L_{-2}+\frac{3}{2(2 h+1)} L_{-1}^{2}\right]|h\rangle \text {. }
$$

This state is null if

$$
h=\frac{1}{16}[5-c \pm \sqrt{(c-1)(c-25)}] .
$$

The correlator of any field with this null state should vanish. This condition gives a new differential equation for the conformal blocks. This equation can be solved for a certain class of theories called minimal models. The two-dimensional conformal field theories, therefore, are based on a different computational formalism from the conventional approach which can give non-perturbative solutions for the quantum field theories.

\subsection{Unitarity in Classification}

The two-dimensional conformal field theories are classified by three input parameters, $c, h_{i}$ and $C_{i j k}$. The problem of deciding all possible sets of parameters is called the classification problem. The solution for the problem has fundamental importance for both string theories and two-dimensional statistical systems. By taking vacuum expectation valucs for (1.4), $c \geq 0$ for a positive semi-definite Hilbert space.

For a given Verma module of a highest weight $|h\rangle$, the unitarity condition that all decendents should have positive definite norm can provide strong restriction on possible values of $c$ and $h$. For a given level $N$, there are $P(N)$ number of decendent states where physical states are given as linear combinations of positive norm. $P(N)$ denotes the number of partitions of integer $N$. The determinant of the $P(N) \times P(N)$ inner product matrix has been computed by Ka.c..$^{[55]}$ The formula is

$$
\operatorname{det} M_{N}(c, h)=\alpha_{N} \prod_{p q \leq N}\left(h-h_{p, q}(c)\right)^{P(N-p q)}
$$

where $h_{p, q}(c)$ is a positive number given as a function of $c$ and integers $p, q$. By 
excluding the region where the determinant becomes negative, one can classify the theory completely for $0 \leq c<1$ by discrete series of allowed values of $c$ and $h^{[17]}$ This is called the unitary minimal series and the allowed values are

$$
c=1-\frac{6}{m(m+1)} \quad m=3,4, \ldots
$$

with allowed primary fields of conformal dimension for given $m$ are given by

$$
h_{p, q}(m)=\frac{[(m+1) p-m q]^{2}-1}{4 m(m+1)}
$$

where integers $p, q$ are given as $1 \leq p \leq m-1,1 \leq q \leq p$. The Verma module of $\left|h_{p, q}\right\rangle$ has a null state at level $p q$.

For $c \geq 1$, the determinant becomes positive definite if the conformal dimension $h \geq 0$. Therefore, we need another principle for these class of theories for complete classification. Most of all, there must exist extended conformal symmetries because the number of primaries with respect to the Virasoro algebra is infinite for $c \geq 1$. If the number of primary fields are finite, the theories are called rational conformal field theories. Recent progress shows that complete classification for the rational conformal field theories may be possible by studying modular transformation properties of characters defined on torus.

\subsection{MOdUlar Invariance on the TORUS}

Partition functions of conformal field theories on the torus are defined as

$$
Z(\tau, \bar{\tau})=q^{-c / 24} \bar{q}^{-\bar{c} / 24} \operatorname{Tr} q^{L_{0}} \bar{q}^{L_{0}}=\sum_{h, \bar{h}} N_{h, \bar{h}} \chi_{h}(q) \bar{\chi}_{\bar{h}}(\bar{q})
$$

We use short notation $q=\exp (2 \pi i \tau)$ with the modular parameter $\tau$ and $N_{h, \bar{h}}$ are nonnegative integers. The Virasoro character $\chi_{h}$ is defined as

$$
\chi_{h}(q) \equiv q^{-c / 24} \operatorname{Tr}_{h} q^{L_{0}}=q^{-(c / 24)+h} \sum_{n=0}^{\infty} d_{h}(n) q^{n}
$$

where $d_{h}(n)$ is equal to the degeneracy of states in the representation at level $n$. Since the trace is defined over all decendents of the primaries, we should not 
include the null states and their decendents in the trace. If there is no null state, $d_{h}(n)=P(n)$ and the character is,

$$
\chi_{h}(q)=q^{-c / 24+h} \sum_{n} P(n) q^{n}=q^{-(c-1) / 24+h} \eta(q)^{-1}
$$

where

$$
-\eta(q) \equiv q^{1 / 24} \prod_{k=1}^{\infty}\left(1-q^{k}\right)
$$

For the minimal models, we should subtract the contribution from the Verma module generated by the null state at level $p q$. Since each null state of the minimal model has its corresponding null state, we should add and subtract these modules alternately to avoid overcounting:

$$
\chi_{p q}(q)=q^{-(c-1) / 24} \eta(q)^{-1} \sum_{k=-\infty}^{\infty}\left[q^{h_{2 m k+p, q}}-q^{h_{2 m k+p,-q}}\right] .
$$

Modular transformations on the torus are generated by two fundamental transformations $T$ and $S ; T: \tau \rightarrow \tau+1$ and $S: \tau \rightarrow-1 / \tau$. The partition functions (1.17) must be invariant under the modular trasformations. Under the $T$,

$$
T: \chi_{h}(q) \rightarrow e^{2 \pi i(h-c / 24)} \chi_{h}(q)
$$

and by $S$

$$
S: \chi_{h}(q) \rightarrow \chi_{h}(\widetilde{q})=\sum_{h^{\prime}} S_{h, h^{\prime}} \chi_{h^{\prime}}(q)
$$

where $h^{\prime}$ runs over all primary fields in the theory and $\widetilde{q}$ denotes $\exp (-2 \pi i / \tau)$. The matrix $S_{h, h^{\prime}}$, referred to S-transformation matrix, must satisfy unitary condition $S S^{\dagger}=S^{\dagger} S=1$ for the inverse $S$ transformation. Also, $S$ and $T$ satisfy $(S T)^{3}=$ $1, S^{2}=C(C$ denotes complex conjugate coming from time reversal). The 
S-transformation matrix must be symmetric. ${ }^{[13]}$ For the minimal models, the Stransformation matrix is

$$
S_{p q}^{p^{\prime} q^{\prime}}=\left(\frac{8}{m(m+1)}\right)^{1 / 2}(-1)^{(p+q)\left(p^{\prime}+q \prime\right)} \sin \left(\pi \frac{p p^{\prime}}{m}\right) \sin \left(\pi \frac{q q^{\prime}}{m+1}\right)
$$

The modular invariance of the partition function gives a condition on the matrix with nonnegative integer elements $N_{h, \bar{h}}$ :

$$
S N S^{\dagger}=N
$$

and on the conformal dimensions of the primaries :

$$
N_{h, \bar{h}}=0 \quad \text { if } \quad h-\bar{h} \neq \text { integer } \quad \text { for } \quad c=\bar{c}
$$

Finding the solutions for (1.25) is very important both for the classification of conformal field theories and in understanding of operator contents of the theories. The only complete solutions found up to now are for the minimal models and $S U(2)$ Kač-Moody algebra. ${ }^{[18,19]}$ In these cases, the solutions are known as $A-D-E$ classifications. The original nomenclature was motivated by the close relationship with the classification of simply-laced Lie algebras. Class $A$ denotes the diagonal form $N_{h, \bar{h}}=\delta_{h, \bar{h}}$, which is obvious from the unitarity of S-transformation matrix. Class $D$ includes the partition functions with off-diagonal form of $N_{h, \bar{h}}=\delta_{h, \pi(\bar{h})}$ with a permutation $\pi$. Class $E$ contains three special modular invariants which do not belong to class $A$ or $D$. The operators of class $A$ are all spinless fields while class $D$ and $E$ contain nonzero integer-spin fields.

Modular invariance can play important role in the classification of conformal field theories. If there are null states, the number of decendents of level $n$ should be reduced, or $d_{h}(n) \leq P(n)$. Therefore, if $q$ and $\widetilde{q}$ are purely real, we get an 
inequality

$$
\chi_{h}(q) \leq q^{-(c-1) / 24+h} \eta(q)^{-1} .
$$

By taking the limit of $\operatorname{Im} \tau \rightarrow 0^{+}$or $q \rightarrow 1$ and $\widetilde{q} \rightarrow 0$, the right-hand of (1.27) becomes $\eta(q \rightarrow 1)^{-1}$ and, using the identity $\eta(\widetilde{q})=(\operatorname{Im} \tau)^{1 / 2} \eta(q)$,

$$
\eta(q \rightarrow 1)^{-1}=\lim _{\tilde{q} \rightarrow 0}(\operatorname{Im} \tau)^{1 / 2} \widetilde{q}^{-1 / 24}
$$

Then, the partition function has the upper limit of

$$
Z \leq \mid \mathfrak{q}^{-1 / 12} \operatorname{Im} \tau \sum_{h, \bar{h}} N_{h, \bar{h}}
$$

While from the modular invariance of $Z$ and (1.17), as $\widetilde{q} \rightarrow 0, Z=Z(\widetilde{q}, \widetilde{q}) \cong$ $\widetilde{q}^{-(c+\bar{c}) / 24}$ from the fact that the most dominant contribution to (1.17) comes from the identity primary field with conformal dimension 0 . Therefore, the condition (1.29) becomes

$$
|\widetilde{q}|^{-(c+\bar{c}) / 24} \leq|\widetilde{q}|^{-1 / 12} \operatorname{Im} \tau \sum_{h, \bar{h}} N_{h, \bar{h}}
$$

If $(c+\bar{c}) / 2 \geq 1$ or, especially $c \geq 1$ for the case of $c=\bar{c}$, only solution for (1.30) is $\sum_{h, \bar{h}} N_{h, \bar{h}}=\infty$. In other words, we need an infinite number of Virasoro primaries for the conformal field theories with $c \geq 1{ }^{[9]}$ However, we may introduce a larger infinite-dimensional algebra so that there may exist a finite number of primary fields with respect to this algebra. In next section, we will explain one typical example of this extended algebra.

If one deals with rational conformal field theories with finite number of primaries, ultimate classification may be possible by investigating the matrix $S$ using the results of E. Verlinde. ${ }^{[10]}$ The story starts with fusion rule algebra, which describes a selection rule for the operator product expansions. The fusion rule matrix element $\mathcal{N}_{i j}{ }^{k}$ is defined as the number of different ways to fuse the primaries that is 
consistent with the operator product expansion (1.9). If $C_{i j k}=0$, then $\mathcal{N}_{i j}{ }^{k}=0$. Verlinde's observation is that the modular transformation $S: \tau \rightarrow-1 / \tau$ diagonalizes the fusion rules. Therefore, for a given $\mathcal{N}_{i j}{ }^{k}$ from $\phi_{i} \times \phi_{j}=\sum_{k} \mathcal{N}_{i j}{ }^{k} \phi_{k}$ one can find possible S-transformation matrix element $S_{i j}$ as eigenvectors of $\mathcal{N}_{i j}{ }^{k}$. Using the identities $S^{2}=C$ and $(S T)^{3}=1$, all possible values of $(c, h)$ can be classified. Furthermore, one can get new modular invariant partition functions out of known ones using fusion rule automorphisms which preserve operator algebras under renaming of primary fields. ${ }^{[2,13]}$ We want to emphasize again that the modular invariance on the torus, which is an one-loop consistency condition for string field theory, is very important both for the determination of operator contents and for the complete classification of rational conformal field theories. 


\section{Kač-Moody Algebra ${ }^{[7,8]}$}

In the previous section, we showed that conformal ficld theories with $c \geq 1$ can be rational only when the chiral algebra is extended from the Virasoro algebra. Every primary field with respect to the new chiral algebra $\mathcal{A}$ creates highest-weight state representation of the algbra $\mathcal{A}$ by acting on the vacuum. From the result of the previous section, it can be shown that a single $\mathcal{A}$ representation contains an infinite number of Virasoro primaries. We can formulate conformal field theories with extended algebras in exactly the same fashion as with Virasoro algebra.

Among the extended algebras, Kač-Moody algebra is particularly interesting as emphasized in the previous section. Mathematically, Kač-Moody algebra comes as an extension of a finite Lie algebra by adding an extra simple root to the finite roots. Physically, this algebra is realized as a continuous symmetry of the WessZumino-Witten models on group manifolds. The Wess-Zumino-Witten action is given by

$$
S=\frac{k}{16 \pi} \int d^{2} x \operatorname{Tr}\left[\partial_{\alpha} g^{-1} \partial_{\alpha} g\right]+\Gamma
$$

where the Wess-Zumino term $\Gamma$ is given by

$$
\Gamma=\frac{k}{24 \pi} \int d^{3} y \epsilon^{i j k} \operatorname{Tr}\left[g^{-1} \partial_{i} g g^{-1} \partial_{j} g g^{-1} \partial_{k} g\right]
$$

where the parameter $k$, called the level, must be integer for the consistency of Wess-Zumino term. This action describes the motion of string on group manifold $G$. If this manifold is taken to form some extra dimension of a string theory, the group $G$ becomes the gauge group of the theory in space-time. The finite group $G$ must be extended to the affine algebra for describing local symmetry of two-dimensional world-sheet. In addition to string theories, Wess-Zumino-Witten models are also useful for statistical models.

The action (2.1) is classically scale invariant. Quantum mechanically, it gives rise to a conformal field theory, whose central charge depends on the level $k$ and 
the finite group $G$. The action is also invariant under the transformation

$$
g(z, \bar{z}) \rightarrow \Omega(z) g(z, \bar{z}) \bar{\Omega}(\bar{z})
$$

where $\Omega \in G$. Here $z$ and $\bar{z}$ are two-dimensional complex coordinates. The WessiZumino term is essential for the invariance. This $G \times \bar{G}$ local symmetry is the Kać-Moody symmetry. The symmetry is generated by conserved currents $J^{a}(z)$ and $\bar{J}^{a}(\bar{z})$. They are defined as

$$
\begin{aligned}
& J(z)=J^{a}(z) \tau^{a}=-\frac{k}{2}\left(\partial_{z} g\right) g^{-1} \\
& \bar{J}(\bar{z})=\bar{J}^{a}(\bar{z}) \tau^{a}=-\frac{k}{2}\left(\partial_{\bar{z}} g^{-1}\right) g
\end{aligned}
$$

where the $\tau^{a}$ 's are antihermitian generators of the finite algebra $G$ nomalized by $\operatorname{Tr} \tau^{a} \tau^{b}=-\frac{1}{2} \delta_{a b}$. With energy-momentum tensor $T(z)$ and $\bar{T}(\bar{z})$, the currents (2.4) generate the full chiral algebra $\mathcal{A} \times \overline{\mathcal{A}}$ which contains the Virasoro algebra as a subalgebra. In addition to operator product between $T$ 's in (1.4), one can find operator products as follows,

$$
\begin{aligned}
T(z) J^{a}(w) & \sim \frac{1}{(z-w)^{2}} J^{a}(w)+\frac{1}{z-w} \partial_{w} J^{a}(w) \\
J^{a}(z) J^{b}(w) & \sim \frac{k \delta^{a b}}{(z-w)^{2}}+\frac{f^{a b c}}{z-w} J^{c}(w) .
\end{aligned}
$$

Using the mode $J_{n}^{a}$ from $J_{n}^{a}=\oint d z z^{n} J^{a}(z)$ and the modes $L_{n}$ 's from $T(z)$, we find the commutation relations for $\mathcal{A}$,

$$
\begin{aligned}
& {\left[L_{n}, J_{m}^{a}\right]=-m J_{n+m}^{a}} \\
& {\left[J_{n}^{a}, J_{m}^{b}\right]=f^{a b c} J_{n+m}^{c}+\frac{k}{2} n \delta^{a b} \delta_{n+m, 0} .}
\end{aligned}
$$

Of course, similar relations hold for the anti-holomorphic generators $\bar{L}_{n}$ and $\bar{J}_{n}^{a}$. From the action (2.1), we can find that the energy-momentum tensor is given as a 
Sugawara form with the second Casimir of the adjoint representation $c_{v}$,

$$
T(z)=-\frac{1}{k+c_{v}}: J^{a}(z) J^{a}(z):
$$

In terms of the modes:

$$
L_{n}=-\frac{1}{k+c_{v}} \sum_{m=-\infty}^{\infty} \because J_{m}^{a} J_{n-m}^{a}:
$$

Then, the central charge and conformal dimension of a primary field of a representation $\lambda$ of $G$ are given by

$$
c=\frac{k \operatorname{dim} G}{k+c_{v}} \quad h_{\lambda}=\frac{c_{\lambda}}{k+c_{v}}
$$

where $c_{\lambda}$ is the second Casimir of the $\lambda$ representation. If the vacuum is invariant under the extended symmetry, we can find an extra Ward identity corresponding to the Kač-Moody algebra in addition to (1.8),

$$
\sum_{k=1}^{n} \tau_{i_{k}}^{a}\left\langle\phi_{i_{1}}\left(z_{1}, \bar{z}_{1}\right) \cdots \phi_{i_{n}}\left(z_{n}, \bar{z}_{n}\right)\right\rangle=0
$$

This equation plus the previous informations detcrmines the four-point correlation functions completely in terms of the input parameters $k$ and $f^{a b c}$, through the following procedure: We can find a null state for the Kač-Moody algebra which can be used to construct additional differential equations. The null state is derived by acting $\left[\kappa L_{-1}-J_{-1}^{a} J_{0}^{a}\right]$ on a highest weight state. This state can lead to the differential equation known as Knizhnik-Zamoldchikov equation, ${ }^{[7]}$

$$
\left[\kappa \partial_{z_{i}}-\sum_{j \neq i}^{n} \frac{\tau_{i}^{a} \tau_{j}^{a}}{z_{i}-z_{j}}\right]\left\langle\phi_{1}\left(z_{1}\right) \cdots \phi_{n}\left(z_{n}\right)\right\rangle=0
$$

where $\kappa=-\left(k+c_{v}\right) / 2$. 
The primary fields $\phi_{i}$ 's of Kač-Moody algebra generate the highest-weight state $\left|\lambda_{i}\right\rangle,\left|\lambda_{i}\right\rangle \equiv \phi_{i}(0)|0\rangle$, with respect to both Virasoro and finite algebra $G$. One of great advantages of Kač-Moody algebra is that characters for the highest weights are given by the well-known formula for characters of affine Lie algebras due to Weyl and Kač. Since $L_{0}$ is added to the Cartan subalgebra, our definition of character (1.18) is consistent with the specialized Weyl-Kać formula with some vanishing parameters. The difficulty in expressing the character of a given highest-weight state explicitly arises from the problem of finding null states systematically. It is particularly difficult for conformal field theories with extended symmetries because of their complicated structures. The reason why the Weyl-Kač formula is powerful is that one can sum the allowed decendents systematically using the extended Weyl group. The character is expressed as a certain sum over Weyl group:

$$
\chi_{\Lambda}=\frac{\sum_{w \in W} \epsilon(w) e^{w(\Lambda+\rho)-\rho}}{\sum_{w \in W} \epsilon(w) e^{w(\rho)-\rho}}
$$

where the affine Weyl group $W$ is a semidirect product of the finite Weyl group $\dot{W}$ and translation group $T$ in the root lattice $M$. The constant $\rho$ is half the sum of positive roots. Furthermore, we can express the sum over $M$ using theta functions whose modular transformation properties are well-known. The formal definition of the theta function is

$$
\Theta_{\lambda}=e^{-|\lambda|^{2} \delta / 2 k} \sum_{t \in T} e^{t(\lambda)}
$$

where $\delta$ is the imaginary root of the Kač-Moody algebra. Therefore, the specialized character is given by

$$
\chi_{\Lambda}(0, \tau, 0)=e^{2 \pi i \tau s_{\Lambda}} \frac{\sum_{w \in \dot{W}} \epsilon(w) \Theta_{w(\Lambda+\rho)}(0, \tau, 0)}{\sum_{w \in \dot{W}} \epsilon(w) \Theta_{w(\rho)}(0, \tau, 0)},
$$

where the number $s_{\Lambda}$ is given by

$$
s_{\Lambda}=\frac{|\Lambda+\rho|^{2}}{2\left(k+h^{\vee}\right)}-\frac{|\rho|^{2}}{2 h^{\vee}} .
$$

We use here the dual Coxeter number $h^{\vee}$ which is equal to the second Casimir $c_{v}$. 
Using the standard formula of Lie algebra, one finds

$$
s_{\Lambda}=\frac{c_{\Lambda}}{k+h^{\vee}}-\frac{1}{24} \frac{k \operatorname{dim} G}{k+h^{\vee}}=h_{\Lambda}-\frac{c}{24}
$$

as one expects from the definition of a character.

The modular transformations of the characters can be derived from those of the theta function.

$$
\begin{aligned}
& \left.\Theta_{\lambda}\right|_{S}=(-i \tau)^{1 / 2}\left|M^{*} / k M\right|^{-1 / 2} \sum_{\mu \in P_{k}} \exp \left[-\frac{2 \pi i}{k}(\lambda \mid \mu)\right] \Theta_{\mu} \\
& \left.\Theta_{\lambda}\right|_{T}=\exp \left[\frac{\pi i}{k}(\lambda \mid \lambda)\right] \Theta_{\lambda} \ldots
\end{aligned}
$$

$M^{*}$ denotes the weight lattice and $P_{k}$ is the set of allowed integer affine weights for a given level $k$. By applying these equations to (2.14), the S-transformation matrix element is given by

$$
S_{\bar{\lambda}_{, \bar{\mu}}}=(-i)^{|\dot{\Delta}|}\left|M^{*} /\left(k+h^{\vee}\right) M\right|^{-1 / 2} \sum_{w \in \dot{W}} \epsilon(w) \exp \left[-\frac{2 \pi i}{k+h^{\vee}}(w(\bar{\lambda}) \mid \bar{\mu})\right],
$$

where $\bar{\lambda}=\bar{\Lambda}+\bar{\rho}$ and $\bar{\Lambda}$ is the highest weight of the finite Lie algebra $G$. One can check that the matrix $S$ is unitary. Although we derived explicit formulae for the modular transformations, solutions of (1.25) for the modular invariant partition functions are not easy to find. Only for $S U(2)$, are the complete solutions known. ${ }^{[19]}$ In the next section, wc are going to find a set of modular invariants for nonsimplyconnected group manifolds defined by orbifold constructions. This approach can be extended toward a complete classification of the conformal field theories with extended chiral algebras.

We want to close this section with additional remarks on the possible applications of Kač-Moody algebras. The coset construction of $G / H$ models with any 
subgroup $H$ of $G$ can describe many interesting known conformal field theories. Using the fact that, ${ }^{[23]}$

$$
\left[L_{n}^{G / H}, L_{m}^{G / H}\right]=\left[L_{n}^{G}, L_{m}^{G}\right]-\left[L_{n}^{H}, L_{m}^{H}\right]
$$

the central charge of $G / H$ is given by $c^{G / H}=c^{G}-c^{H}$. The Hilbert space $\mathcal{H}(\Lambda, \lambda)$ of the coset conformal field theory is defined by the relation

$$
\mathcal{H}(\Lambda)=\sum_{\lambda} \mathcal{H}(\lambda) \otimes \mathcal{H}(\Lambda, \lambda)
$$

where $\mathcal{H}(\Lambda)$ and $\mathcal{H}(\lambda)$ denote the Hilbert space of $G$ and $H$, respectively. The case of $c^{G / H}=0$ describes the equivalence of two conformal field theories of group manifolds. The condition can be satisfied only if the level of $G$ equals 1 . In this case, $H$ is called a conformal subalgebra, and we should conclude that the lower algebra $H$ is extended to the higher algebra $G$. If $c^{G / H} \neq 0$, we have a new model which we can relate to other known theories. For example, the coset model based on

$$
\frac{S U(2)_{p} \times S U(2)_{q}}{S U(2)_{p+q}}
$$

describes the unitary minimal series when $p=1$ and its supersymmetric version when $p=2$. In similar way, parafermionic theories are described by the coset models of $S U(n)$. Furthermore, the characters built on $\mathcal{H}(\Lambda, \lambda)$, referred to branching functions can have direct physical meanings in two-dimensional lattice models where order parameters of the models are obtained from the theta function identities. ${ }^{[25]}$ Therefore, by understanding the modular invariant partition functions of general group manifolds, we can understand the modular transformaion structures of the coset models. 


\section{Modular Invariant Partition Functions from Orbifolds}

Wess-Zumino-Witten models ${ }^{[7,8]}$ are prototypical rational conformal field theories. The classification of rational conformal field theories has been the focus of much recent attention. But even the small subclass consisting of Wess-ZuminoWitten models is not well understood; all consistent models are not known.

One powerful restriction is modular invariance. For example, the states of the theory must be such that the one loop partition function is modular invariant. A list of possible modular invariant partition functions has been compiled and proven complete only for the simplest case, that of $S U(2) .^{[19]}$

Remarkably, the $S U(2)$ partition functions may be labelled by the simplylaced Lie algebras, i.e. those of class $A, D$ and $E$. There are the trivial diagonal modular invariants (class $A$ ) and also exceptional ones (class $E$ ) occurring for isolated values of $\mathrm{Kac}-\mathrm{Moody}$ central charge $k$. The remaining modular invariants (class $D$ ) are the partition functions for strings propagating on the group manifold $S O(3){ }^{[8,56]}$ So besides the trivial and exceptional, all $S U(2)$ modular invariants are partition functions for strings on nonsimply-connected group manifolds. If this pattern continues for other Lie groups, strings on nonsimply-connected group manifolds are certainly important.

Felder, Gawedzki and Kupiainen ${ }^{[58]}$ have studied the canonical quantization of Wess-Zumino-Witten models. Using the geometry of line bundles over the loop groups of $G$, they derive consistent spectra for arbitrary nonsimply-connected groups $G=\tilde{G} / B$, where $\tilde{G}$ is the covering group, and $B$ a subgroup of its center $B(\tilde{G})$. In this letter we use the orbifold ${ }^{[59]}$ approach advocated in reference 8 to construct the partition functions, thus providing a simple confirmation of their results.

The crucial mathematical relation we use is the isomorphism between the outer automorphism group $O(\hat{g})$ of the (untwisted) Kač-Moody algebra $g$ and the center

$\star$ This was conjectured for $S U(3)$ in reference 57 . 
$B(\tilde{G})$, and its relation to the modular transformations of the torus. Bernard ${ }^{[60]}$ has shown that in the space of characters of highest weight representations of $\hat{g}$, it is the modular tranformation $S(\tau \rightarrow-1 / \tau)$ that transforms an element $A \in O(\hat{g})$ into an element $\alpha \in B(\tilde{G})$, and vice versa. He and others ${ }^{[61]}$ have used this fact to derive many modular invariants. These are now understood to be some, but not all, of the partition functions for strings on nonsimply-connected group manifolds.

A Wess-Zumino-Witten model one-loop partition function is a sesquilinear combination of specialized characters

$$
\chi_{\lambda}(\tau)=\operatorname{tr}_{\lambda} e^{2 \pi i\left(L_{0}-\frac{c}{24}\right) \tau}
$$

of highest weight representations of a Kać-Moody algebra $\hat{g}$. Here $\lambda=\lambda_{\mu} \omega^{\mu}=$ $\left(\lambda_{0}, \bar{\lambda}\right)$ is the highest weight of the corresponding representation, $\lambda \in \mathbf{Z}$, and $\omega^{\mu}$ are the fundamental affine weights. $\bar{\lambda}$ is a highest weight of the finite Lie algebra given. For a unitary representation, we must have $\lambda_{\mu} k^{\vee \mu}=k$, where $k$ is the Kač-Moody central charge and $k^{\vee \mu}$ are the dual Kač labels.

In particular, the partition function for strings propagating on the nonsimplyconnected group manifold $G=\tilde{G} / B, B \subset B(\tilde{G})$, is of the form

$$
Z(\tilde{G} / B)=\sum_{\lambda^{\prime}, \lambda} \chi_{\lambda^{\prime}}^{*} N_{\lambda^{\prime} \lambda} \chi_{\lambda}
$$

It can also be written as an orbifold ${ }^{[59]}$ partition function. ${ }^{[8]}$ If $\sigma_{1}+\sigma_{2} \tau$ are the coordinates of the torus and $\tau$ its modulus, we let $\left(\alpha_{1}, \alpha_{2}\right)$ denote the contribution to the partition function from fields obeying the twisted boundary conditions

$$
\begin{aligned}
& \phi\left(\sigma_{1}+2 \pi, \sigma_{2}\right)=\alpha_{1} \phi\left(\sigma_{1}, \sigma_{2}\right) \\
& \phi\left(\sigma_{1}, \sigma_{2}+2 \pi\right)=\alpha_{2} \phi\left(\sigma_{1}, \sigma_{2}\right) .
\end{aligned}
$$

Since the boundary conditions are defined for real coordinates, and not for complex coordinates, it is not clear how the chiral structure of the original theory will change

$\dagger$ Many of these may also be derived from the branching rules for the conformal embedding $\hat{s u}(p)^{q} \otimes \hat{s u}(q)^{p} \subset \hat{s} \hat{u}(p q)^{1} !^{\mid 62]}$ 
by orbifolding. Actually, using $z=\exp \left[\left(\sigma_{1}+\sigma_{2}\right) i\right]$ and $\bar{z}=\exp \left[\left(\sigma_{1}-\sigma_{2}\right) i\right]$ one finds that the holomorphic and anti-holomorphic coordinates are invariant under the twist. Understanding chiral structures of orbifold conformal field theories is a very difficult subject. ${ }^{[63]}$ One way to understand the chiral structure is to find modular invariant partition functions expressed with holomorphic and anti-holomorphic characters. To do this, we derive the partition function by adding untwisted and twisted sectors consistently. The guiding principle is modular invariance. Later, we express the partition function using the characters.

The partition function can be written as

$$
Z(\tilde{G} / B)=\frac{1}{|B|} \sum_{\substack{\alpha_{1}, \alpha_{2} \in B \\\left[\alpha_{1}, \alpha_{2}\right]=0}}\left(\alpha_{1}, \alpha_{2}\right)
$$

where $|B|$ is the order of $B$. The modular invariance of this expression is guaranteed, since under any transformation $\tau \rightarrow(a \tau+b) /(c \tau+d),(a d-b c=1 ; a, b, c, d \in \mathbf{Z})$, $\left(\alpha_{1}, \alpha_{2}\right)$ transforms to $\left(\alpha_{1}^{d} \alpha_{2}^{c}, \alpha_{1}^{b} \alpha_{2}^{a}\right)^{[59,64]}$ If $B=\mathbf{Z}_{N},(3.4)$ reduces to

$$
Z\left(\tilde{G} / \mathbf{Z}_{N}\right)=\frac{1}{N} \sum_{m, n=0}^{N-1}\left(\alpha_{1}^{m}, \alpha_{2}^{n}\right)
$$

The trivial example is the partition function on the simply connected group manifold $\tilde{G}:^{[8]}$

$$
N(\tilde{G})_{\lambda^{\prime} \lambda}=(1,1)_{\lambda^{\prime} \lambda}=\delta_{\lambda^{\prime} \lambda}
$$

Untwisted fields are those obeying (3.3) with $\alpha_{1}=1$. The contribution to (3.5) from the untwisted sector is denoted $Z_{1}$ :

$$
Z_{1}\left(\tilde{G} / \mathbf{Z}_{N}\right)=\frac{1}{N} \sum_{n=0}^{N-1}\left(1, \alpha^{n}\right)
$$

Using these last two objects and the generators $S(\tau \rightarrow-1 / \tau)$ and $T(\tau \rightarrow \tau+1)$ of the modular group, it is in principle possible to obtain the full partition function 
$Z\left(\tilde{G} / \mathbf{Z}_{N}\right){ }^{[8]}$ The following formula is valid for $N$ prime:

$$
Z\left(\tilde{G} / \mathbf{Z}_{N}\right)=\left[1+\sum_{v=0}^{N-1} T^{v} S\right] Z_{1}\left(\tilde{G} / \mathbf{Z}_{N}\right)-Z(\tilde{G})
$$

For $N$ not prime, the situation is more complicated. For example, one can verify

$$
Z\left(\tilde{G} / \mathbf{Z}_{4}\right)=\left[1+\sum_{v=0}^{3} T^{v} S\right] Z_{1}\left(\tilde{G} / \mathbf{Z}_{N}\right)-Z(\tilde{G})-\frac{1}{2} Z\left(\tilde{G} / \mathbf{Z}_{2}\right)
$$

The $\mathbf{Z}_{2}$ group of the last term is generated by $\alpha^{2}$ if $\alpha$ generates $\mathbf{Z}_{4}$. For general $N$ not prime, we expect subtraction of terms proportional to $Z\left(\tilde{G} / \mathbf{Z}_{p}\right)$ for $p \mid N$ would be necessary. For simplicity, we therefore restrict to $N$ prime, and use $(3.8)$.

The $T$ transformation is of quite simple form:

$$
T_{\lambda^{\prime} \lambda}=\delta_{\lambda^{\prime} \lambda} \exp \left\{\frac{\pi i|\lambda+\rho|^{2}}{k+h^{\vee}}-\frac{\pi i|\rho|^{2}}{h^{\vee}}\right\}
$$

Here $\rho=\sum_{\mu} \omega^{\mu}$ and the dual Coxeter number is $h^{\vee}=\sum_{\mu} k^{\vee \mu} \omega^{\mu}$. But the dimension of the $S$-transformation matrix grows rapidly with $k$, and the expression for its. elements involves a sum over the Weyl group of $g$. So explicitly constructing the $S$-transformation matrix is extremely tedious. This is the main obstruction to using formulae like (3.8) to derive orbifold partition functions.

However, identities proved by Bernard ${ }^{[60]}$ allow us to bypass this difficulty. Consider an element $A$ of the outer automorphism group $O(\hat{g})$ of $\hat{g}$ acting on a highest weight $\lambda$ for a given value of the Kač-Moody central charge $k\left(\lambda_{\mu} k^{\mu}=\right.$ $k$ ). (An example is the generator of $O(\operatorname{su}(N))$, which permutes the fundamental

* This is a significant restriction only for $g=A$ (where $g$ is the Lie algebra of $G$ ). The first interesting case excluded is $S U(4) . \mathbf{Z}_{N}$ with $N$ prime covers all other cases, except for half the possibilities with $g=D$. 
weights as follows: $A \omega^{\mu}=\omega^{\mu+1} ; \omega^{N} \equiv \omega^{0}$.) Restricting to the weight lattice of the finite Lie algebra $g$, one can write

$$
\overline{A(\lambda+\rho)}=\left(k+h^{\vee}\right) \omega^{A(0)}+w_{A}(\overline{\lambda+\rho}) .
$$

Here $\bar{\kappa}=\sum_{i \neq 0} \kappa_{i} \omega^{i}$ is the restriction of an affine weight $\kappa$ to the $g$ weight lattice, $\omega^{A(0)}=A \omega^{0}$, and $w_{A}$ is an element of the Weyl group of $g$ acting in the following way:

$$
w_{A}\left(\omega^{i}\right)=\omega^{A(i)}-k^{\vee i} \omega^{A(0)}
$$

For $\hat{s u}(N)$, it is straightforward to check that $w_{A}$ is given by

$$
w_{A}=w_{\alpha_{1}} w_{\alpha_{2}} \cdots w_{\alpha_{N-1}}
$$

Substituting (3.11) to (2.18), we get an overall phase factor from the first term on the right-hand side of (3.11). Instead of summing over $w$ in (2.18), we can sum over $w^{\prime}=w w_{A}$ because $w^{\prime}$ covers the same set of elements of Weyl group as $w$. Finally, using $\epsilon\left(w w_{A}\right)=\epsilon(w) \epsilon\left(w_{A}\right)$, we obtain

$$
S_{A(\lambda) \lambda^{\prime}}=S_{\lambda \lambda^{\prime}} \epsilon\left(w_{A}\right) \exp \left\{2 \pi i\left(\omega^{A(0)} \mid \lambda^{\prime}+\rho\right)\right\}
$$

Here $\epsilon\left(w_{A}\right)$ is the signature of $w_{A}$; i.e. $\epsilon=+1(-1)$ for the product of an even (odd) number of reflections. Now for all outer automorphisms $A$, we have ${ }^{[58]}$

$$
\epsilon\left(w_{A}\right)=\exp \left\{2 \pi i\left(\omega^{A(0)} \mid \rho\right)\right\}=\exp \left\{\pi i h^{\vee}\left|\omega^{A(0)}\right|^{2}\right\}
$$

So (3.14) reduces to

$$
S_{A(\lambda) \lambda^{\prime}}=S_{\lambda \lambda^{\prime}} \exp \left\{2 \pi i\left(\omega^{A(0)} \mid \lambda^{\prime}\right)\right\}
$$

This last equation is the starting point. Considering it with $A$ replaced by $A^{r}$ 
yields

$$
\left(\omega^{A^{r}(0)} \mid \lambda\right)=r\left(\omega^{A(0)} \mid \lambda\right) \bmod 1
$$

implying

$$
N\left(\omega^{A(0)} \mid \lambda\right)=0 \bmod 1
$$

if $A^{N}=1$. So we see that the phase on the right hand side of (3.16) is an $N$ th root of unity. In fact, it is the eigenvalue of an element of $B(\tilde{G})$ of order $N$. So, as mentioned above, the modular transformation $S$ maps elements of $O(\hat{g})$ into elements of $B(\tilde{G})$.

Now the untwisted sector partition function $Z_{1}\left(\tilde{G} / \mathbf{Z}_{N}\right)$ is built from the diagonal partition function $Z(\tilde{G})$ by projecting onto $Z_{N}$ invariant states (compare (3.7) and (3.5)). So

$$
\begin{aligned}
N_{1}\left(\tilde{G} / \mathbf{Z}_{N}\right)_{\lambda^{\prime} \lambda} & =\delta_{\lambda^{\prime} \lambda} \frac{1}{N} \sum_{r=0}^{N-1} \exp \left\{2 \pi i r\left(\omega^{A(0)} \mid \lambda\right)\right\} \\
& =\delta_{\lambda^{\prime} \lambda} \delta_{1}\left\{\left(\omega^{A(0)} \mid \lambda\right)\right\}
\end{aligned}
$$

where we have defined

$$
\delta_{1}(x)= \begin{cases}1, & \text { if } x=0 \bmod 1 \\ 0, & \text { otherwise }\end{cases}
$$

Using (3.16) it is then easy to show

$$
S N_{1}\left(\tilde{G} / \mathbf{Z}_{N}\right)_{\lambda^{\prime} \lambda}=\frac{1}{N} \sum_{r=0}^{N-1} \delta_{\lambda^{\prime} A^{r}(\lambda)}
$$

Applying successive $T$-transformations yields

$$
T^{v} S N_{1}\left(\tilde{G} / \mathbf{Z}_{N}\right)_{\lambda^{\prime} \lambda}=\frac{1}{N} \sum_{r=0}^{N-1} \delta_{\lambda^{\prime} A^{r}(\lambda)} \exp \left\{-2 \pi i v\left[\frac{\left|A^{r}(\lambda+\rho)\right|^{2}-|\lambda+\rho|^{2}}{2\left(k+h^{\vee}\right)}\right]\right\} .
$$


Since $k^{\vee A(0)}=1$

$$
\left(\omega^{A(0)} \mid w(\nu)\right)=\left(\omega^{A(0)} \mid \nu\right) \bmod 1
$$

for any element $w$ of the Weyl group of $g$, and any integral weight $\nu$. This and equations (3.11) and (3.15) simplify (3.22) to

$$
T^{v} S N_{1}\left(\tilde{G} / \mathbf{Z}_{N}\right)_{\lambda^{\prime} \lambda}=\frac{1}{N} \sum_{r=0}^{N-1} \delta_{\lambda^{\prime} A^{r}(\lambda)} \exp \left\{-2 \pi i v\left[\left(\omega^{A^{r}(0)} \mid \lambda\right)+\frac{k}{2}\left|\omega^{A^{r}(0)}\right|^{2}\right]\right\} .
$$

... Twisting a string by $\alpha^{N}=1$ must make no difference. Replacing $v$ with $v+N$ in (3.24) therefore demands

$$
\frac{N k}{2}\left|\omega^{A^{r}(0)}\right|^{2}=0 \bmod 1
$$

for all $r$. This can be simplified, however, since

$$
\frac{N k}{2}\left|\omega^{A(0)}\right|^{2}=0 \bmod 1
$$

is sufficient to ensure (3.25) and furthermore that

$$
\frac{k}{2}\left(\omega^{A^{r}(0)} \mid \omega^{A^{s}(0)}+\omega^{A^{t}(0)}\right)=\frac{k}{2}\left(\omega^{A^{r}(0)} \mid \omega^{A^{s+t}(0)}\right) \bmod 1
$$

Equation (3.26) disallows certain integer values of Kać-Moody central charge $k$. For $\hat{s} u(N)$, since $\left|\omega^{A(0)}\right|^{2}$ is the form of integer $/ N,(3.26)$ restricts the level $k$ to be even integer. It was derived in reference 58 by requiring consistency of the Wess-Zumino term on a torus, with one of its cycles mapped into a nontrivial closed path in $G$. Thus it is a consequence of the nontrivial fundamental group $\pi_{1}\left(G=\tilde{G} / \mathbf{Z}_{N}\right)=\mathbf{Z}_{N}$. 
Substituting (3.24) into the general formula (3.8), and using (3.27), we finally obtain

$$
\begin{aligned}
& N\left(\tilde{G} / \mathbf{Z}_{N}\right)_{\lambda^{\prime} \lambda}=N_{1}\left(\tilde{G} / \mathbf{Z}_{N}\right)_{\lambda^{\prime} \lambda}+ \\
& \sum_{r=1}^{N-1} \delta_{\lambda^{\prime} A^{r}(\lambda)} \frac{1}{N} \sum_{v=0}^{N-1} \exp \left\{-2 \pi i r\left[\left(\omega^{A^{v}(0)} \mid \lambda+\frac{k}{2} \omega^{A^{r}(0)}\right)\right]\right\}
\end{aligned}
$$

Since $N$ is prime the factor $r$ outside the square brackets may be dropped, and we can write

$$
\ldots N\left(\tilde{G} / \mathbf{Z}_{N}\right)_{\lambda^{\prime} \lambda}=\frac{1}{N} \sum_{m, n=0}^{N-1} \delta_{\lambda^{\prime} A^{m}(\lambda)} \exp \left\{-2 \pi i\left(\omega^{A^{n}(0)} \mid \lambda+\frac{k}{2} \omega^{A^{m}(0)}\right)\right\}
$$

This is exactly the form found by Felder, Gawedzki and Kupiainen. ${ }^{[58\}}$ Furthermore, it is easy to convince oneself that

$$
\left(\alpha^{m}, \alpha^{n}\right)=\delta_{\lambda^{\prime} A^{m}(\lambda)} \exp \left\{-2 \pi i\left(\omega^{A^{n}(0)} \mid \lambda+\frac{k}{2} \omega^{A^{m}(0)}\right)\right\} .
$$

This result shows how each twisted sector has chiral structure.

The condition (3.26) guarantees the integrality of the elements of the matrix $Z\left(\tilde{G} / \mathbf{Z}_{N}\right)$. This must be, since these quantities count the numbers of primary fields. We may rewrite the final result in a way that manifests this property:

$$
N\left(\tilde{G} / \mathbf{Z}_{N}\right)_{\lambda^{\prime} \lambda}=\sum_{m=0}^{N-1} \delta_{\lambda^{\prime} A^{m}(\lambda)} \delta_{1}\left\{\left(\omega^{A(0)} \mid \lambda+\frac{k m}{2} \omega^{A(0)}\right)\right\}
$$

We should emphasize here that (3.29) is a modular invariant for all cases, whether or not it is integer valued. But it reduces to the physically sensible partition function (3.31) with integral values only when the Kač-Moody central charge $k$ obeys (3.26).

* The authors of reference 58 also considered the unique semi-simple possibility for $\tilde{G}$ simple: $B=\mathbf{Z}_{2} \times \mathbf{Z}_{2}$ for $g=D_{l}, l$ even. 
We will now write the partition function in a more compact notation, and use it to verify modular invariance. Considering an outer automorphism $A$ as acting on the space of highest weights of unitary representations, we have

$$
A_{\lambda^{\prime} \lambda}=\delta_{\lambda^{\prime} A(\lambda)}
$$

Then (3.16) becomes in matrix notation

$$
A S \doteq S \alpha
$$

where $\alpha \in B(\tilde{G})$ is of course diagonal.

$$
\alpha_{\lambda^{\prime} \lambda}=\delta_{\lambda^{\prime} \lambda} \exp \left[2 \pi i\left(\omega^{A(0)} \mid \lambda\right)\right]
$$

Thus the modular transformation $S$ diagonalises the outer automorphisms of $\hat{g}$.

If we have another related pair $A^{\prime} \in O(\hat{g}), \alpha^{\prime} \in B(\tilde{G})$, i.e. $A^{\prime} S=S \alpha^{\prime}$, we define

$$
\begin{aligned}
& A^{\prime} \circ \alpha=A^{\prime} \alpha \exp \left[+\pi i k\left(\omega^{A^{\prime}(0)} \mid \omega^{A(0)}\right)\right] \\
& \alpha \circ A^{\prime}=\alpha A^{\prime} \exp \left[-\pi i k\left(\omega^{A(0)} \mid \omega^{A^{\prime}(0)}\right)\right]
\end{aligned}
$$

so that

$$
A^{\prime} \circ \alpha=\alpha \circ A^{\prime}
$$

Then the partition function may be written simply as

$$
Z\left(\tilde{G} / \mathbf{Z}_{N}\right)=\frac{1}{N} \sum_{m, n=0}^{N-1} A^{m} \circ \alpha^{n}
$$

If $C$ is the charge conjugation matrix, we have

$$
S^{2}=C, \quad C A=A^{-1} C
$$


so that (3.33) also implies

$$
S^{\dagger} \alpha S=A^{-1} .
$$

Therefore

$$
S Z\left(\tilde{G} / \mathbf{Z}_{N}\right)=\frac{1}{N} \sum_{m, n} \alpha^{m} \circ A^{-n}=Z\left(\tilde{G} / \mathbf{Z}_{N}\right)
$$

Finally, it is straightforward to prove

$$
T^{\dagger}\left(A \circ \alpha^{\prime}\right) T=A \circ \alpha \alpha^{\prime}
$$

establishing the $T$-invariance of $Z\left(\tilde{G} / \mathbf{Z}_{N}\right)$. 


\section{Further Remarks}

In reference 13 , it has been shown that unless $N_{\lambda \bar{\lambda}}$ is diagonal or a permutation matrix $\left(\delta_{\lambda, \pi(\bar{\lambda})}\right)$, the chiral algebra of the theory extends to a higher one. Furthermore, the existence of nondiagonal theorics can always be understood either in terms of the existence of automorphisms of the fusion rule algebra, or in terms of an enhancement of the chiral algebra. For the fully extended algebra, the only nondiagonal modular invariants come from automorphisms of the fusion rule algebra. The fusion rule automorphism is defined as a permutation of primary fields which leaves the fusion coefficients $\mathcal{N}_{i j}{ }^{k}$ invariant.

We can classify our results (3.31) according to the above results. Using the condition in (3.31)

$$
\delta_{1}\left\{\left(\omega^{A(0)} \mid \lambda+\frac{k m}{2} \omega^{A(0)}\right)\right\},
$$

we can select an appropriate integer $m$ for a given weight $\lambda$. Then, the permutation is given as $\pi=A^{m}$. We can easily prove that this permutation of highest weights is the automorphism of fusion rule algebra. By applying Eq. (3.16) to Verlinde's relation $^{[10]}$

$$
\mathcal{N}_{i j}{ }^{k}=\sum_{n} \frac{S_{i n} S_{j n} S^{\dagger n k}}{S_{0 n}}
$$

one can see that the phase factors cancel in this expression to give

$$
\mathcal{N}_{i j}{ }^{k}=\mathcal{N}_{\pi(i) \pi(j)}{ }^{\pi(k)}
$$

However, some of the modular invariants in (3.31) are not associated with fusion rule automorphisms. These cases arise when (4.1) is satisfied irrespective of $m$. For $S U(N)$, this is satisfied if level $k=p \cdot N$ with some integer $p$. Then, the 
condition reduces to

$$
\delta_{1}\left\{\left(\omega^{A(0)} \mid \lambda\right)\right\}
$$

and the partition function will be the form of

$$
Z=\sum_{\lambda \in M}\left|\chi_{\lambda}+\cdots+\chi_{A^{N-1}(\lambda)}\right|^{2}+N\left|\chi_{\lambda_{0}}\right|^{2}
$$

where $M$ is set of the highest weights which satisfy (4.4) and are not related by outer automorphism $A$ to another. As long as the order of the center $N$ is prime, all the weights generated from a weight $\lambda$ in $M$ by applying $A$ successively are different from another in the orbit, except for one special weight $\lambda_{0}$ given by $[p, p, \cdots, p]$ which satisfies $A\left(\lambda_{0}\right)=\lambda_{0}$. Since the matrix $N_{\lambda, \lambda^{\prime}}$ for (4.5) is not a permutation matrix, the chiral algebra is extended to a higher algebra. From the point of view of this extended algebra, the partition function (4.5) is a diagonal form with the characters $\chi_{\Lambda}$ defined by

$$
\chi_{\Lambda}=\chi_{\lambda}+\cdots+\chi_{A^{N-1}(\lambda)}
$$

and $N \chi_{\lambda_{0}}$ 's which may represent different primary states of the higher algebra. Using this partition function, we may find exceptional modular invariants which are neither of diagonal nor permutational form. If there exists an automorphism of fusion rules of the extended algebra, we can find an additional nondiagonal modular invariant partition function. Since this partition function, as a combination of the original characters, cannot be obtained from (3.31), this should give a new modular invariant partition function for the original algebra.

To find the fusion rule automorphism of the extended algebra, we try to find relation similar to (3.16) for the matrix $S$ of the extended algebra. The matrix $S$ for the extended algebra is completely determined by that of the original algebra because the phase factor $\exp \left[2 \pi i\left(\omega^{A(0)} \mid \lambda\right)\right]$ in (3.16) disappears as far as $\lambda$ 
satisfies (4.4). Therefore, we can get a new permutation $\pi$ by finding a pair $\left(\lambda, \lambda^{\prime}\right)$ such that

$$
S_{\lambda \mu}=S_{\lambda^{\prime} \mu} \text { for all } \mu \in M
$$

The permutation is given by the relation $\pi(\lambda)=\lambda^{\prime}$. By computing the matrix $S$ numerically for $S U(N)$, we have found two well-known exceptional partition functions: ${ }^{[1,57]} S U(2)^{k=16}$ and $S U(3)^{k=9}$. For these solutions, one highest weight in the pair comes from the special weight $\lambda_{0}$. But we did not find any other exceptional modular invariants of $S U(N)$ from this method.

The simple structure of Eq. (3.31) may exist in a more general class of rational conformal field theories, perhaps those obtained by the coset construction. ${ }^{[23]}$ Since the characters of the coset models are given as branching functions defined by

$$
\chi_{\Lambda}(\tau)=\sum_{\lambda} \Phi_{\Lambda}^{\lambda}(\tau) \chi_{\lambda}(\tau)
$$

where $\chi_{\Lambda}, \chi_{\lambda}$ are the characters of $G$ and $H$ respectively, we can find new modular invariants for the coset model from modular invariant partition functions for $G$ and $H$. The new modular invariant partition functions are given by

$$
Z_{G / H}=\sum_{\Lambda, \Lambda^{\prime}, \lambda, \lambda^{\prime}} N_{\Lambda, \Lambda^{\prime}} M_{\lambda, \lambda^{\prime}} \Phi_{\Lambda}^{\lambda} \Phi_{\Lambda^{\prime}}^{\lambda^{\prime *}}
$$

with two matrices $N_{\Lambda, \Lambda^{\prime}}, M_{\lambda, \lambda^{\prime}}$ from (3.31). However, this expression may not be physically acceptable for two reasons.$^{[65]}$ First, many pairs of weights $(\Lambda, \lambda)$ may not appear in the spectrum of the coset theory. Second, some states may be labelled by several pairs $(\Lambda, \lambda)$ which should be identified. Although these two problems (selection rules and field identification) may be solved rather easily for the special coset models considered in the reference 65 , this is much more difficult for a general coset theory.

Using the formalism presented in section 3 , it is possible to solve these problems for the cases in which $G$ and $H$ are Kač-Moody algebras. This analysis will be 
presented in reference 66 . There we will present a projection matrix $\hat{F}$ to find the relationship between the outer automorphisms of $G$ and $H$. Since the modular transformation matrix $S$ for the coset model is obtained as a direct product of two $S$ matrices of $G$ and $H$, if the phase factors in Eq. (3.16) for $G$ and $H$ cancel each other we can identify two fields in the coset models. Since the projection matrix $\hat{F}$ applies to the highest weights of $G$ to produce those of $H$, the outer automorphism $A$ of $G$ and $a$ of $H$ is related by

$$
\hat{F} A=a \hat{F}
$$

Since the phase depends on the nature of the automorphisms, the field identification for a general coset model can be obtained by studying the projection matrix. 


\section{REFERENCES}

1. B. W. Lee, C. Quigg, and H. B. Thacker, Phys. Rev. D16 (1977) 1519.

2. M. S. Chanowitz, M. A. Furman, and I. Hinchliffe, Nucl. Phys. B153 (1979) 402.

3. J. M. Cornwall, D. N. Levin and G. Tiktopoulos, Phys. Rev. D10 (1974) 1145. Erratum: ibid, D11 (1975) 972.

4. C. Ahn, M. E. Peskin, B. W. Lynn, and S. Selipsky, Nucl. Phys. B309 (1988) 221.

5. A. M. Polyakov, Phys. Lett. 103B (1981) 207, 211.

6. A. A. Belavin, A. M. Polyakov, and A. B. Zamolodchikov, Nucl. Phys. B241 (1984) 333.

7. E. Witten, Comm. Math. Phys. 92 (1984), 455; S.P. Novikov, Usp. Mat. Nauk 37 (1982), 3; V. Knizhnik and A.B. Zamolodchikov, Nucl. Phys. B247 (1984), 83 .

8. D. Gepner and E. Witten, Nucl. Phys. B278 (1986), 493.

9. J. L. Cardy, Nucl. Phys. B270 (1986) 186.

10. E. Verlinde, Nucl. Phys. B300 (1988) 360.

11. C. Vafa, Phys. Lett. 206B (1988) 421.

12. R. Dijkgraaf and E. Verlinde (Utrecht U.), THU-88/25 (1988).

13. G. Moore and N. Seiberg, Nucl. Phys. B313 (1989) 16.

14. G. Moore and N. Seiberg, Phys. Lett. 212B (1988) 451, IASSNS-HEP-88/39 (1988).

15. H. Sonoda, Nucl. Phys. B311 (1988) 417.

16. V. Kac and D. Peterson, Adv. Math. 53 (1984) 125.

17. D. Friedan, Z. Qiu, and S. Shenker, Phys. Rev. Lett. 52 (1984) 1575. 
18. D. Gepner, Nucl. Phys. B287 (1987) 111.

19. A. Cappelli, C. Itzykson and J.-B. Zuber, Nucl. Phys. B280[FS18] (1987), 445; Comm. Math. Phys. 113 (1987), 1; A. Kato, Mod. Phys. Lett. A2 (1987), 585 .

20. D. Friedan, Z. Qiu and S. Shenker, Phys. Lett. 151B (1985) 33.

21. V. A. Fateev and A. B. Zamolodchikov, ZhETF 89 (1985) 380.

22. V. A. Fateev and A- B. Zamolodchikov, Nucl. Phys. B280[FS18] (1987) 644.

... 23. P. Goddard, A. Kent, and D. Olive, Phys. Lett. 152B (1985), 88.

24. D. Kastor, E. Martinec, and Z. Qiu, Phys. Lett. 200B (1988) 434.

25. E. Date, M. Jimbo, A. Kuniba, T. Miwa, and M. Okado, Nucl. Phys. B290[FS20] (1987) 231.

26. S. D. Mathur, S. Mukhi, and A. Sen, Phys. Lett. 213B (1988) 303.

27. C. Ahn and M. A. Walton, Phys. Lett. 223B (1989) 343.

28. M. Veltman, Phys. Lett. 91B (1980) 95.

29. W. J. Marciano and A. Sirlin, Phys. Rev. D29 (1984) 945; Phys. Rev. D31 (1985) 213; Phys. Rev. Lett. 46 (1981) 163.

30. A. Sirlin, Phys. Rev. D22 (1980) 971.

31. B. W. Lynn and R. G. Stuart, Nucl. Phys. B253 (1985) 216.

32. D. C. Kennedy and B. W. Lynn, SLAC-PUB-4039 (1988).

33. B. W. Lynn, M. E. Peskin and R. G. Stuart, in Tests of Electroweak Theories, B. W. Lynn and C. Verzegnassi, eds. (World Scientific, Singapore, 1986).

34. T. Applequist and J. Carazzone, Phys. Rev. D11 (1975) 2856.

35. J. Collins, F. Wilczek, and A. Zee, Phys. Rev. D18 (1978) 242.

36. W. Alles, Ch. Boyer, A. Buras, Nucl. Phys. B119 (1977) 125. 
37. O. P. Sushkov, V. V. Flambaum, and I. B. Khriplovich, Sov. J. Nucl. Phys. 20 (1975) 537.

38. M. Kuroda, F. M. Renard and D. Schildknecht, Phys. Lett. 183B (1987) 366.

39. M. Lemoine and M. Veltman, Nucl. Phys. B164 (1980) 445.

40. R. Philippe, Phys. Rev. D26 (1982) 1588.

41. B. Grzadkowski and'Z. Hioki, Phys. Lett. 197B (1987) 213.

42. P. Kalyniak and M. K. Sundaresan, Carleton Univ. preprint Print-87-0800 (1987).

43. M. Bohm, A. Denner, T. Sack, W. Beenakker, F. Berends, H. Kuijf, Wurzburg Univ. preprint Print-88-0073 (1987).

44. K. Hagiwara, R. D. Peccei, D. Zeppenfeld, and K. Hikasa, Nucl. Phys. B262 (1987) 233.

45. D. C. Kennedy, B. W. Lynn, and C. J.-C. Im, SLAC-PUB-4128 (1988).

46. M. Veltman, Nucl. Phys. B123 (1977) 89.

47. U. Amaldi, A. Bohm, L. S. Durkin, P. Langacker, A. K. Mann, W. J. Marciano, A. Sirlin, H. H. Williams, Phys. Rev. D36 (1987) 1385.

48. G. Passarino and M. Veltman, Nucl. Phys. B160 (1979) 151.

49. G. 't Hooft and M. Veltman, Nucl. Phys. B153 (1979) 365.

50. B. W. Lynn, Ph.D. thesis, Columbia University (1982); University Microfilms.

51. R. G. Stuart, Max Planck Institute preprint MPI-PAE/PTh 85/87 (1987).

52. M. S. Chanowitz and M. K. Gaillard, Nucl. Phys. B261 (1985) 379.

C. E. Vayonakis, Lett. Nuovo Cimento 17 (1976) 383.

G.J. Gounaris, R. Kogerler, and H. Neufeld, Phys. Rev. D34 (1986) 3257.

53. R. G. Stuart, D. Phil. thesis, Oxford University (1985). 
54. B. W. Lynn, SLAC-PUB-3358 (1984).

55. V. Kač, Lecture Notes in Physics 94 (1979) 441.

56. A. Abouelsaood and D. Gepner, Phys. Lett. 176B (1986), 380.

57. P. Christe and F. Ravanini, Int. J. Mod. Phys. A4 (1989), 897.

58. G. Felder, K. Gawedzki and A. Kupiainen, Comm. Math. Phys. 117 (1988), 127.

59. L.Dixon, J. Harvey, C. Vafa and E. Witten, Nucl. Phys, B261 (1985), 678; B274 (1986), 286.

-60. D. Bernard, Nucl. Phys. B288 (1987), 628.

61. D. Altschuler, J. Lacki and P. Zaugg, Phys. Lett. 205B (1988), 281.

62. M.A. Walton, preprint SLAC-PUB-4680, to be published in Nucl. Phys. B.

63. R. Dijkgraaf, C. Vafa, E. Verlinde, and H. Verlinde, HUTP-88/A052 and THU-88/38.

64. C. Vafa, Nucl. Phys. B273 (1986), 592.

65. D. Gepner, Princeton Univ. preprint PUPT-1114.

66. C. Ahn and M. A. Walton, SLAC-PUB-5067 (1989) 